\title{
Common littoral pycnogonids of the Mediterranean Sea
}

\author{
Tobias Lehmann ${ }^{1,2}$, Martin Heß $\aleph^{2,3}$, Roland R. Melzer ${ }^{1,2,3}$ \\ 1 Bavarian State Collection of Zoology-SNSB, Münchhausenstraße 21, 81247 Munich, Germany \\ 2 Ludwig-Maximilians-Universität München, Department Biology II, Großhaderner Straße 2, 82152 Planegg-Martinsried, Germany \\ 3 GeoBio-CenterLMU, Richard-Wagner-Straße 10, 80333 Munich, Germany
}

http://zoobank.org/6EBE944E-00E5-473A-94CE-4DF4C54E54D8

Corresponding author: Tobias Lehmann (lehmann@zsm.mwn.de)

Received 14 March 2014

Accepted 2 October 2014

Published 31 October 2014

Academic editor:

Carsten Lüter

\section{Key Words}

\section{SEM}

Pycnogonida

Sea spider

Achelia

Ammothella

Anoplodactylus

Callipallene

Endeis

Pycnogonum

Tanystylum

Trygaeus

\begin{abstract}
In the present study 21 littoral pycnogonid species from 5 families are analysed: Ammotheidae ( 9 species), Callipallenidae ( 5 species), Endeidae ( 2 species), Phoxichilidiidae ( 3 species), and Pycnogonidae ( 2 species). The material was mainly taken from Mediterranean pycnogonid collections housed in the Bavarian State Collection of Zoology. Additional material was collected during field trips. Altogether the material was obtained from six different locations: Banyuls-sur-Mer (France), Tavolara Island (Italy), Elba Island (Italy), Giglio Island (Italy), Sicily Island (Italy), and Istria Peninsula (Croatia). The animals were studied in detail with a scanning electron microscope (SEM). Additionally series of light microscopic pictures were made to generate extended depth of field pictures of whole animals. The observed features are compared with previous literature.
\end{abstract}

\section{Introduction}

The Pycnogonida or sea spiders are exclusively marine invertebrates, numbering more than 1330 species worldwide (Arango and Wheeler 2007, Bamber and El Nagar 2013). They were first named Pycnonides Latreille, 1810. Later they were given the names Podosomata Leach, 1815 and Pantopoda Gerstaeker, 1863. The latter name is still in use for the order which comprises all extant pycnogonids. Although largely unnoticed, due to their cryptic form, often small size, and economic insignificance, sea spiders are common benthic animals occurring from the littoral zone to the deep-sea, in all oceans from the poles to the tropics. Most species are benthic, few are in- terstitial, some are pelagic and some are commensals or ectoparasites of other invertebrates. Pycnogonids are normally small animals; littoral species have a leg span of at most a few centimetres, while polar and deep-water species can achieve a leg span of $70 \mathrm{~cm}$ (Ruppert et al. 2004).

The phylogenetic position of the Pycnogonida has long been controversial and is still under debate. Today pycnogonids are placed either within the Chelicerata as sister taxon of the Euchelicerata or as sister taxon of all other Euarthropoda (Dunlop and Arango 2005, Regier et al. 2010, Giribet and Edgecombe 2012). The phylogenetic relationships within the group are discussed as well. Traditionally the Pycnogonida were divided into eight families (Hedgpeth 1947) but with uncertain relationships. 
Recently studies on this point, using morphological and / or molecular characters were realized (Arango 2002, Arango 2003, Arango and Wheeler 2007, Nakamura et al. 2007). On the basis of these studies Bamber (2007) and Bamber and El Nagar (2013) suggested 11 families.

The Mediterranean Sea as we know it today developed about 5 million years ago. After the Messinian salinity crisis (5.6 to 5.3 Mya) the Mediterranean Sea was filled in a major flood approximately 5.3 million years ago, in which water poured in from the Atlantic Ocean and through the Strait of Gibraltar (Tichy et al. 2001). Consequently, the Mediterranean marine biota including the pycnogonid fauna is derived primarily from the Atlantic Ocean. The opening of the Suez Canal in 1869 created the first salt-water passage between the Mediterranean and Red Sea allowing some Red Sea species to invade the Mediterranean (lessepsian migration) (Schmidt et al. 2001). Anoplodactylus californicus, A. digitatus, and Pigrogromitus timsanus are regarded as being lessepsian migrants (Chimenz-Gusso and Lattanzi 2003).

In the Mediterranean Sea 56 pycnogonid species are known until now. A current species list of the Mediterranean pycnogonid fauna is found in Chimenz-Gusso and Lattanzi (2003), with the completion of Anoplodactylus nanus, described by Krapp, Kocak, and Katagan in 2008 (Krapp et al. 2008). The Mediterranean pycnogonid fauna consists mostly of littoral species, and deep-sea species are an exception. It is assumed that, because of the geological barrier caused by the Strait of Gibraltar (max. depth $286 \mathrm{~m}$ ) and the temperature barrier in the deep-sea (Atlantic $1-4^{\circ} \mathrm{C}$, Mediterranean $12^{\circ} \mathrm{C}$ ), the deep water fauna in the Mediterranean is relatively species-poor (Schmidt et al. 2001). Deep-sea pycnogonid species adapted to cold water such as representatives of the Colossendeidae and Pallenopsidae are either absent or rarely occur in the Mediterranean. A similar barrier also exists between the western and eastern basin of the Mediterranean and between the Mediterranean Sea and the Black Sea.

Mediterranean pycnogonid literature is scattered (e.g. Giltay 1929, Helfer 1936, Faraggiana 1940, Bourdillon 1954, Stock 1958, Bacescu 1959, Stock 1962, Stock and Soyer 1965, Stock 1966, Zavodnik 1968, Krapp 1973, Schüller 1989, Pérez-Ruzafa and Munilla 1992, Chimenz et al. 1993, Munilla 1993, Munilla and Nieto 1999, Chimenz Gusso 2000, Montoya Bravo et al. 2006, Krapp et al. 2008, Kocak et al. 2010, Kocak and Alan 2013). Of particular significance is Dohrn's (1881) “Die Pantopoden des Golfes von Neapel und der angrenzenden Meeresabschnitte" with its excellent drawings and descriptions of 18 new species. The first detailed comprehensive taxonomic work about sea spiders in the Mediterranean was by Bouvier (1923), which also dealt with Atlantic species. Zoogeographical studies were done by Arnaud (1987) and Chimenz-Gusso and Lattanzi (2003); the deep-water species were discussed by Stock (1987). As many of the species distributed in Mediterranean are of Atlantic origin, also the survey of Bamber (2010) is of special interest.

There is a rapidly growing body of molecular studies especially on the Antarctic and Subantractic fauna (e.g. Mahon et al. 2008, Nielsen et al. 2009, Krabbe et al. 2010, Dietz et al. 2011, Arango et al. 2011, Weis and Melzer 2012b, Dietz et al. 2013, Carapelli et al. 2013). In contrast, there is a lack of publications with molecular studies for the Mediterranean. However, so far - as at January 2014 - 11 Mediterranean pycnogonid species are listed in BOLD (Ratnasingham and Hebert 2007) and 10 species in GenBank (Benson et al. 2010). Detailed morphological analyses of Mediterranean Sea species are required to support future molecular studies, and specimens stored in natural history collections can provide a useful basis for these studies (see also Dunlop et al. 2007, Weis et al. 2011, Weis and Melzer 2012a).

In the present study representatives of some of the major genera of Mediterranean pycnogonids were sourced from the Bavarian State Collection of Zoology. Additional material was collected during field trips. A significant objective of this study is to remove any ambiguity in species identifications by providing a pictorial atlas principally based on high resolution Scanning Electron Microscope (SEM) images. Classification follows Bamber (2007) and PycnoBase (Bamber and El Nagar 2013).

\section{Material and methods}

Material: The majority of specimens were sourced from the Bavarian State Collection of Zoology. Additional material was collected during field trips to Banyuls-sur-Mer, France (June/July 2006) and Rovinj, Croatia (September 2006 and May 2007). Collecting sites are summarized in Figure 1. Details are given under material section of each species. All material was conserved and stored in $75 \%$ ethanol. Preparations were made according to methods described in Bolte (1996). Only adult animals were used for light microscopic and SEM imaging. Species determinations are based on the original descriptions and a variety of literature suitable for Mediterranean pycnogonids (e.g. Dohrn 1881, Bouvier 1923, Stock 1968, Bamber 2010). Synonyms followed PycnoBase (Bamber and El Nagar 2013) and Müller (1993). All specimens (SEM and alcohol material) used for this study are deposited at the Bavarian State Collection of Zoology.

Light microscopy: Light microscopic pictures were taken using an Olympus SZX stereo microscope and a Jenoptic Prog-Res C12 digital camera $(2580 \times 1944$ px; 96 dpi; colour depth 24 bit). Larger specimens (Endeis charybdaea, E. spinosa) were photographed using a Wild-Heerbrugg M5A stereo microscope and a Canon Digital IXUS 850 IS digital camera $(3072 \times 2304$ px; 180 dpi; colour depth 24 bit). Up to 12 pictures with different focus steps along the $\mathrm{z}$-axis were combined to a single respective image with a greater field of depth using the computer software Auto Montage (Syncroscopy) or Helicon Focus (HeliconSoft).

Scanning electron microscopy: For SEM preparation, specimens were dehydrated in a graded acetone series $(70 \%, 80 \%, 90 \%, 10 \mathrm{~min}$. each, plus $3 \times 100 \%, 20 \mathrm{~min}$. 
each) and critical-point-dried in a Baltec CPD 030. Dried specimens were mounted on SEM stubs with self-adhesive carbon stickers and coated with gold on a Polaron Sputter Coater. SEM pictures $(2048 \times 1536$ px; 72 dpi; colour depth 8 bit) were made with a LEO 1430VP at 10-20 kV. Scales were inserted using the measurement utility of the SEM.

Nomenclature: The present study follows the nomenclature in Arnaud and Bamber (1987) and Bamber (2010). Hence, the trunk of a pycnogonid is divided into 4 segments, the cephalon (=segment 1 ) and the segments 2, 3, and 4 . The cephalon carries the proboscis anteroventrally, the ocular tubercle dorsally, and four pairs of extremities: the chelifores above the proboscis, the palps laterally, the ovigers ventrally, and one pair of legs laterally. Segments 2, 3, and 4 each carry one pair of legs laterally. The fourth segment carries the abdomen posterodorsally. Some species do not possess palps or chelifores. Ovigers are present in males (with few exceptions, e.g. Pycnogonum subgen. Nulloviger). In some genera ovigers are reduced or absent in females. The number of articles of the chelifores, palps, and ovigers varies within the systematic groups. Each leg is composed of nine articles: first, second, and third coxa, femur, first and second tibiae, tarsus, propodus, and claw. The first coxa articulates with the lateral process of the trunk while the second coxa carries the genital opening ventrally. In males, the cement glands are on the femur and rarely on other articles. The claw is often flanked by two smaller auxiliary claws. All abbreviations used in the figures are provided in Table 1.

\section{Results}

\section{General remarks}

This study is based on 21 Mediterranean species. In all cases, the major morphological characteristics correspond with published descriptions; exceptions see remarks section of each species.

\section{Classification}

Order Pantopoda Gerstäcker, 1863

Suborder Eupantopodida Fry, 1978

Superfamily Ascorhynchoidea Pocock, 1904

Family Ammotheidae Dohrn, 1881

Achelia echinata Hodge, 1864

Achelia langi (Dohrn, 1881)

Achelia vulgaris (Costa, 1861)

Ammothella appendiculata (Dohrn, 1881)

Ammothella biunguiculata (Dohrn, 1881)

Ammothella longipes (Hodge, 1864)

Ammothella uniunguiculata (Dohrn, 1881)

Tanystylum conirostre (Dohrn, 1881)

Trygaeus communis Dohrn, 1881

Superfamily Nymphonoidea Pocock, 1904

Family Callipallenidae Hilton, 1942

Callipallene emaciata (Dohrn, 1881)

Callipallene phantoma (Dohrn, 1881)
Callipallene producta (Sars, 1888)

Callipallene spectrum (Dohrn, 1881)

Callipallene tiberi (Dohrn, 1881)

Superfamily Phoxichilidoidea Sars, 1891

Family Endeidae Norman, 1908

Endeis charybdaea (Dohrn, 1881)

Endeis spinosa (Montagu, 1808)

Family Phoxichilidiidae Sars, 1891

Anoplodactylus angulatus (Dohrn, 1881)

Anoplodactylus petiolatus (Krøyer, 1844)

Anoplodactylus pygmaeus (Hodge, 1864)

Superfamily Pycnogonoidea Pocock, 1904

Family Pycnogonidae Wilson, 1878

Pycnogonum nodulosum Dohrn, 1881

Pycnogonum (Retroviger) pusillum Dohrn, 1881

\section{Ammotheidae Dohrn, 1881 \\ Achelia Hodge, 1864}

\section{Achelia echinata Hodge, 1864}

Figures 2, 5-8

Synonyms: Ammothea brevipes Hodge, 1864

Ammothea echinata Hodge, 1864

Ammothea fibulifera (Dohrn, 1881)

Material. ZSMA20071461: male; Giglio Island, Italy; 08.04.2005; 4-8 m; brown algae. ZSMA20071462: female; Giglio Island, Italy; 11.04.2005; 20 m. ZSMA20071463: female; Rovinj, Croatia; 05.07.2005; 0-1.5 m; Stypocaulon scoparium (Linnaeus) Kützing, 1843.

\section{Achelia langi (Dohrn, 1881)}

Figures 2, 9, 10

Synonyms: Ammothea langi Dohrn, 1881

Material. ZSMA20071464: male; Cape Savudrija, Croatia; 08.10.2004: 0-1 m; under stones. ZSMA20071465: male; Cape Savudrija, Croatia; 22.05.2005: 0-1 m. ZSMA20071466: female; Cape Savudrija, Croatia; 08.08.2005; 0-1 m; on stone.

Remarks. According to Dohrn (1881) the lateral processes 1-3 are armed with one protuberance with spine. Here, in the male, the lateral process 1 has two protuberances with spine and the lateral process 2 and 3 have one (Fig. 9D, E). The lateral processes in the female correspond with Dohrn's description.

\section{Achelia vulgaris (Costa, 1861)}

Figures 2, 11, 12

Synonyms: Achelia franciscana (Dohrn, 1881)

Alcinous vulgaris Costa, 1861

Ammothea fransciscana Dohrn, 1881

Material. ZSMA20071467: male; Bayuls-sur-Mer, France; 07.2004. ZSMA20071468: female; Giglio Island, Italy; 
08.04.2005; 8-13 m; brown algae. ZSMA20071469: female; Giglio Island, Italy; 08.04.2005; 8-13 m; brown algae.

Remarks. Adult males are differentiated by the character described by Dohrn (1881) and Bouvier (1923): three protuberances with spine on each side of coxa 2 . But Dohrn notes that there also exist varieties: two protuberances on each side of coxa 2 or two protuberances on one and three protuberances on the other side of coxa 2.

Here, only coxa 2 of the first leg has three protuberances with spine on the one and two on the other side; the coxa 2 of the other legs has two protuberances with spine on each side (Fig. 11C, D). The other characters of the male, like lateral process not touching each other (Fig. 11B) correspond with the descriptions of Costa (1861), Dohrn (1881) and Bouvier (1923).

\section{Ammothella Verrill, 1900 \\ Ammothella appendiculata (Dohrn, 1881)}

Figures 3, 13, 14

Synonyms: Ammothea appendiculata Dohrn, 1881

Material. ZSMA20071470: female; Cape Savudrija, Croatia; 03.09.2004: 0-1 m. ZSMA20071471: female; Cape Savudrija, Croatia; 08.10.2004: 0-1 m; on stone. ZSMA20071472: female; Cape Savudrija, Croatia; 08.08.2005; 0-1 m; under stone.

Remarks. Dohrn (1881) described the palp with 10 articles; here the palp, as in other Ammothella, has 9 articles (Fig. 13E).

\section{Ammothella biunguiculata (Dohrn, 1881)}

Figures 3, 15, 16

Synonyms: Ammothea biunguiculata Dohrn, 1881

Material. ZSMA20071473: male; Cape Savudrija, Croatia; 08.10.2004: 0-1 m; on stone.

\section{Ammothella longipes (Hodge, 1864)}

Figures 3, 17

Synonyms: Achelia longipes (Hodge, 1864)

Achelia magnirostris (Dohrn, 1881)

Ammothea longipes Hodge, 1864

Ammothella magnirostris (Dohrn, 1881)

Material. ZSMA20071477: female; Bayuls-sur-Mer, France; 07.2004.

\section{Ammothella uniunguiculata (Dohrn, 1881)}

Figures 4, 18

Synonyms: Ammothea uniunguiculata Dohrn, 1881

Material. ZSMA20140003: male; Sicily, Italy; 1988

\section{Tanystylum Miers, 1879}

\section{Tanystylum conirostre (Dohrn, 1881)}

Figures 4, 19-21

Synonyms: Clotenia conirostris Dohrn, 1881

Material. ZSMA20071505: female; Bayuls-sur-Mer, France; 07.2004. ZSMA20071506: female; Bayuls-surMer, France; 07.2004. ZSMA20071507: male; Bayulssur-Mer, France; 07.2004. ZSMA20071508: male; Bayuls-sur-Mer, France; 07.2004.

\section{Trygaeus Dohrn, 1881}

\section{Trygaeus communis Dohrn, 1881}

Figures 4, 22-25

Material. ZSMA20071509: female; Giglio Island, Italy; 12.04.2005. ZSMA20071510: male; Giglio Island, Italy; 12.04.2005. ZSMA20071511: male; Giglio Island, Italy; 12.04.2005. ZSMA20071512: male; Giglio Island, Italy; 12.04.2005. ZSMA20071513: male; Cape Kamenjak, Croatia; 03.05.2007; 0-5 m; Stypocaulon scoparium (Linnaeus) Kützing, 1843.

Remarks. According to Dohrn (1881), Bouvier (1923) and Stock (1966) the palps vary in the number of articles from 4 to 7 and the oviger from 6 to 10 . Here the palp has 7 articles (Fig. 23A) and the male and female oviger have 9 articles (Fig. 23C, D; Fig. 25D). Other specimens not depicted have four palp articles.

\section{Callipallenidae Hilton, 1942 \\ Callipallene Flynn, 1929}

\section{Callipallene emaciata (Dohrn, 1881)}

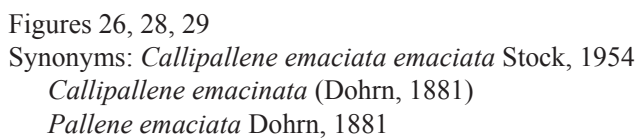

Material. ZSMA20071485: female; Giglio Island, Italy; 09.04.2005; 3-5 m; Halopteris sp.. ZSMA20071486: female; Giglio Island, Italy; 09.04.2005; 3-5 m; Halopteris sp.. ZSMA20071487: male; Giglio Island, Italy; 09.04.2005; 3-5 m; Halopteris sp.

\section{Callipallene phantoma (Dohrn, 1881)}

\footnotetext{
Figures 26, 30-33

Synonyms: Callipallene phantoma crinita Stock, 1952 Callipallene phantoma phantoma (Dohrn, 1881) Pallene phantoma Dohrn, 1881
}

Material.ZSMA20071489: female; Brucoli, Sicily, Italy; 14.09.1988. ZSMA20071490: female; Banyuls-sur-mer, France; 07.2004. ZSMA20071491: female; Giglio Island, Italy; 11.04.2005; $20 \mathrm{~m}$; red algae. ZSMA20071492: male; Giglio Island, Italy; 11.04.2005; 20 m; red algae. 
ZSMA20071493: male; Giglio Island, Italy; 11.04.2005; 20 m; red algae. ZSMA20071494: male; Rovinj, Croatia; 05.07.2005.

Remarks. Dohrn (1881) describes the auxiliary claws as half as long as the claw. According to Stock (1952) the length of auxiliary claws can vary between specimens. Here all 6 specimens from 4 different locations have more or less rudimentary auxiliary claws (Fig. 31F; Fig. 33F, G).

\section{Callipallene producta (Sars, 1888)}

Figures 26, 34, 35

Synonyms: Callipallene brevirostris producta (Sars, 1888)

Pallene producta Sars, 1888

Material. ZSMA20071495: female; Giglio Island, Italy; 08.04.2005; 13-18 m; on brown algae. ZSMA20071496: female; Giglio Island, Italy; 08.04.2005; 13-18 m; on brown algae.

\section{Callipallene spectrum (Dohrn, 1881)}

Figures 27, 36

Synonyms: Callipallene emaciata spectrum (Dohrn, 1881)

Pallene spectrum Dohrn, 1881

Material. ZSMA20140004: male; Banjole, Rovinj, Croatia; 07.1968; $5 \mathrm{~m}$; on Cystoseira sp..

\section{Callipallene tiberi (Dohrn, 1881)}

Figures 27, 37

Synonyms: Callipallene emaciata tiberii (Dohrn, 1881) Pallene tiberi Dohrn, 1881

Material. ZSMA20071488: female; Giglio Island, Italy; 12.04.2005; $1 \mathrm{~m}$; Halopteris sp..

\section{Endeidae Norman, 1908 \\ Endeis Philippi, 1843}

\section{Endeis charybdaea (Dohrn, 1881)}

Figures 38-40

Synonyms: Chilophoxus charybdaeus (Dohrn, 1881)

Phoxichilus charybdaeus Dohrn, 1881

Material. ZSMA20071497: male; Rovinj, Croatia; 04.09.2006; $30 \mathrm{~m}$.

\section{Endeis spinosa (Montagu, 1808)}

Figures 38, 41-44

Synonyms: Chilophoxus spinosus Montagu

Endeis gracilis Philippi, 1843

Endeis laevis (Grube, 1871)

Endeis vulgaris (Dohrn, 1881)

Phallangium spinosum Montagu, 1808

Phoxichilus vulgaris Dohrn, 1881
Material. ZSMA20071498: female; Rovinj, Croatia; 04.2004. ZSMA20071499: male; Rovinj, Croatia; 05.05.2007; 0-2 m; Corallina elongata J.Ellis \& Solander, 1786.

Remarks. According to Dohrn (1881) and Bamber (2010) each lateral process and coxa 1 is armed with one protuberance. Here, in the males the lateral process 1 and the coxa 1 of the first leg has two protuberances (Fig. $42 \mathrm{D}, \mathrm{F})$, the remaining legs correspond with previous observations (e.g. Fig. 42E, G). The females are without such protuberances (Fig. 44C, D).

\section{Phoxichilidiidae Sars, 1891 \\ Anoplodactylus Wilson, 1878}

\section{Anoplodactylus angulatus (Dohrn, 1881)}

Figures 45-47

Synonyms: Anoplodactylus angulirostris (Dohrn, 1881) Phoxichilidium angulatum Dohrn, 1881

Material. ZSMA20071478: female; Banyuls-sur-mer, France; 07.2004. ZSMA20071479: female; Giglio Island, Italy; 14.4.2005; $20 \mathrm{~m}$; red algae.

\section{Anoplodactylus petiolatus (Krøyer, 1844) \\ Figures 45, 48 \\ Synonyms: Anoplodactylus guyanensis Child, 1977 \\ Anoplodactylus hedgpethi Bacescu, 1959 \\ Anoplodactylus longicollis (Dohrn, 1881) \\ Anoplodactylus pygmaeus (Hoek, 1881) \\ Pallene attenuata Hodge, 1864 \\ Phoxichilidium attenuatus (Hodge, 1863) \\ Phoxichilidium longicolle Dohrn, 1881 \\ Phoxichilidium petiolatum Krøyer, 1844}

Material. ZSMA20071480: male; Banyuls-sur-mer, France; 07.2004. ZSMA20042384: male; Bayuls-surMer, France; 06.2003.

Remarks. In literature there is diverse information about the articulation of the oviger, 7 articles in Dohrn (1881) and 6 articles in Bouvier (1923) and King (1986) are reported. Here the ovigers have 6 articles.

\section{Anoplodactylus pygmaeus (Hodge, 1864)}

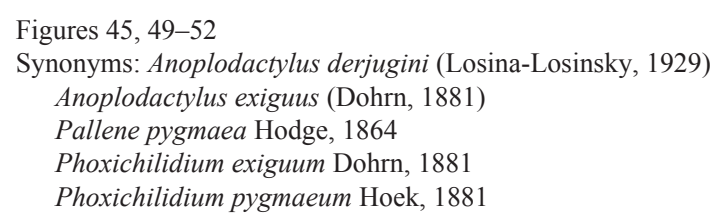

Material. ZSMA20071481: female; Elba, Italy; 20.10.2004; 3-5 m. ZSMA20071482: female; Elba, Italy; 21.10.2004; 3-5 m. ZSMA20071483: male; Elba, Italy; 20.10.2004; 0.5-4 m. ZSMA20071484: male; Cape 
Kamenjak, Croatia; 03.05.2007; 0-5 m; Stypocaulon scoparium (Linnaeus) Kützing, 1843.

Remarks. In the literature there is diverse information about the articulation of the oviger, 7 articles in Dohrn (1881) and 6 articles in Bouvier (1923) and King (1986) are reported. Here the ovigers have 6 articles (Fig. 50E, F).

\section{Pycnogonidae Wilson, 1878}

\section{Pycnogonum Bruennich, 1764}

\section{Pycnogonum nodulosum Dohrn, 1881}

Figures 53,54

Material. ZSMA20071503: female; Banyuls-sur-mer, France; 07.2004; 30 m; red algae. ZSMA20030112: female; Tavolara Island, Sardinia, Italy; 2002.

\section{Pycnogonum (Retroviger) pusillum Dohrn, 1881}

Figures 53, 55

Synonyms: Pycnogonum pusillum Dohrn, 1881
Table 1. Abbreviations used in the figures in alphabetical order.

\begin{tabular}{l|l|l|l}
\hline ab & abdomen & mf & movable finger \\
\hline ac & auxiliary claw & mo & mouth \\
\hline an & anus & ne & neck \\
\hline ce & cephalon & o & oviger-article \\
\hline $\mathbf{c f}$ & chelifore & oc & ocular tubercle \\
\hline $\mathbf{c g}$ & cement gland & ov & oviger \\
\hline $\mathbf{c h}$ & chela & $\mathbf{p}$ & palp-article \\
\hline $\mathbf{c l}$ & claw & $\mathbf{p a}$ & palp \\
\hline $\mathbf{c s}$ & compound spine & $\mathbf{p p}$ & propodus \\
\hline $\mathbf{c u}$ & cutting lamina & $\mathbf{p r}$ & proboscis \\
\hline $\mathbf{c x}$ & coxa & $\mathbf{p t}$ & protuberance \\
\hline $\mathbf{f f}$ & fixed finger & $\mathbf{s b}$ & segment border \\
\hline $\mathbf{f m}$ & femur & $\mathbf{s e}$ & segment \\
\hline $\mathbf{g o}$ & genital openings & $\mathbf{s o}$ & slit organ \\
\hline ha & hair & sp & spine \\
\hline hs & hollow spine & tb & tibia \\
\hline le & leg & te & teeth \\
\hline lp & lateral process & tr & trunk \\
\hline Is & lateral sense organ & ts & tarsus \\
\hline & & &
\end{tabular}

Material. ZSMA20071504: male; Brucoli, Sicily, Italy; 14.09.1988.

\section{Figures}

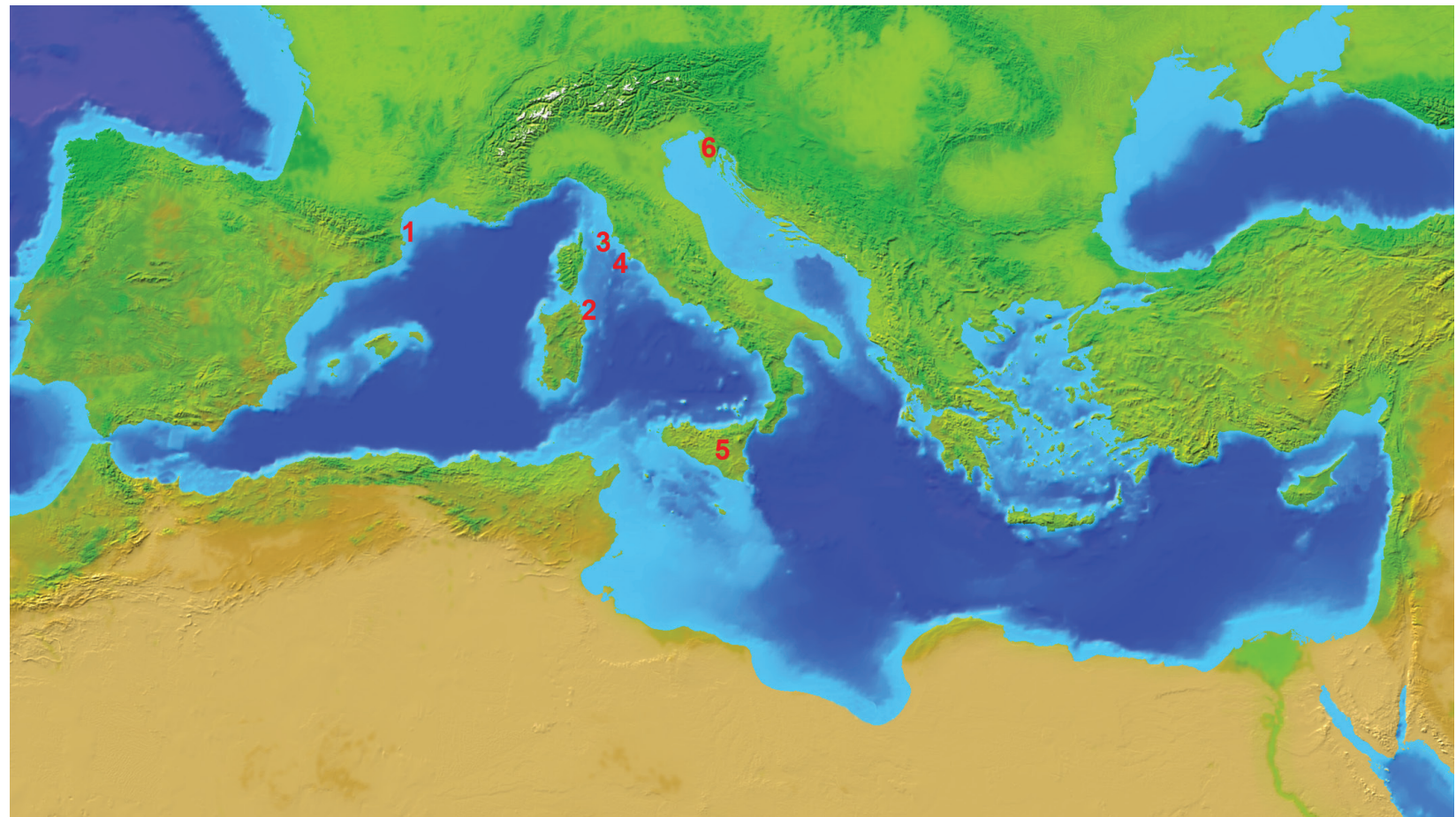

Figure 1. Collecting sites of pycnogonids used in this study: 1: Banyuls-sur-Mer (France); 2: Tavolara Island (Italy); 3: Elba Island (Italy); 4: Giglio Island (Italy); 5: Brucoli, Sicily (Italy); 6: Istria Peninsula (Croatia). 

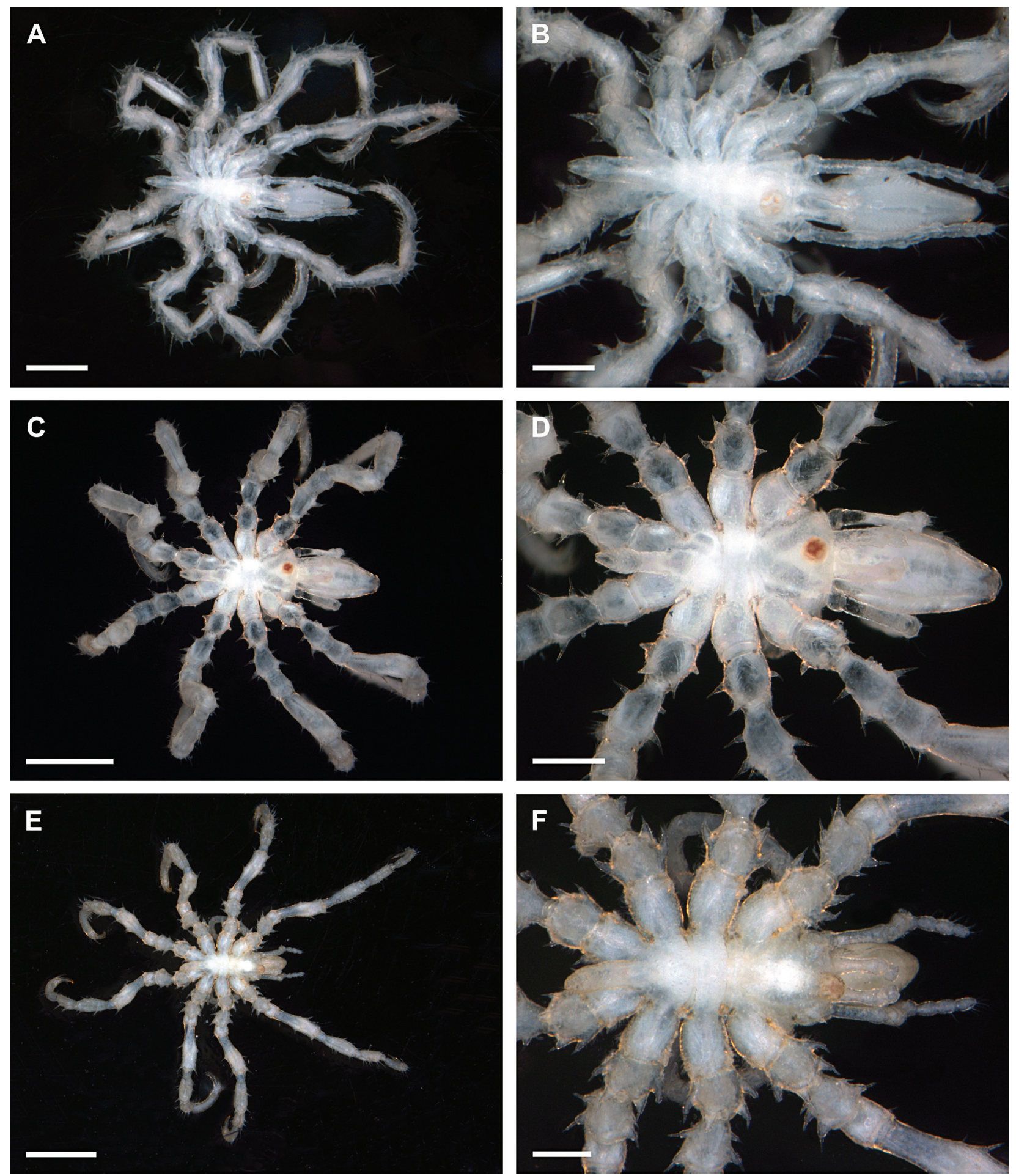

Figure 2. Ammotheidae 1; A, B: Achelia echinata, male, dorsal view; scales $500 \mu \mathrm{m}$ and $250 \mu \mathrm{m}$, respectively; C, D: Achelia langi, male, dorsal view; scales $1 \mathrm{~mm}$ and $500 \mu \mathrm{m}$, respectively; E, F: Achelia vulgaris, male, dorsal view; scales $1 \mathrm{~mm}$ and $250 \mu \mathrm{m}$, respectively. 

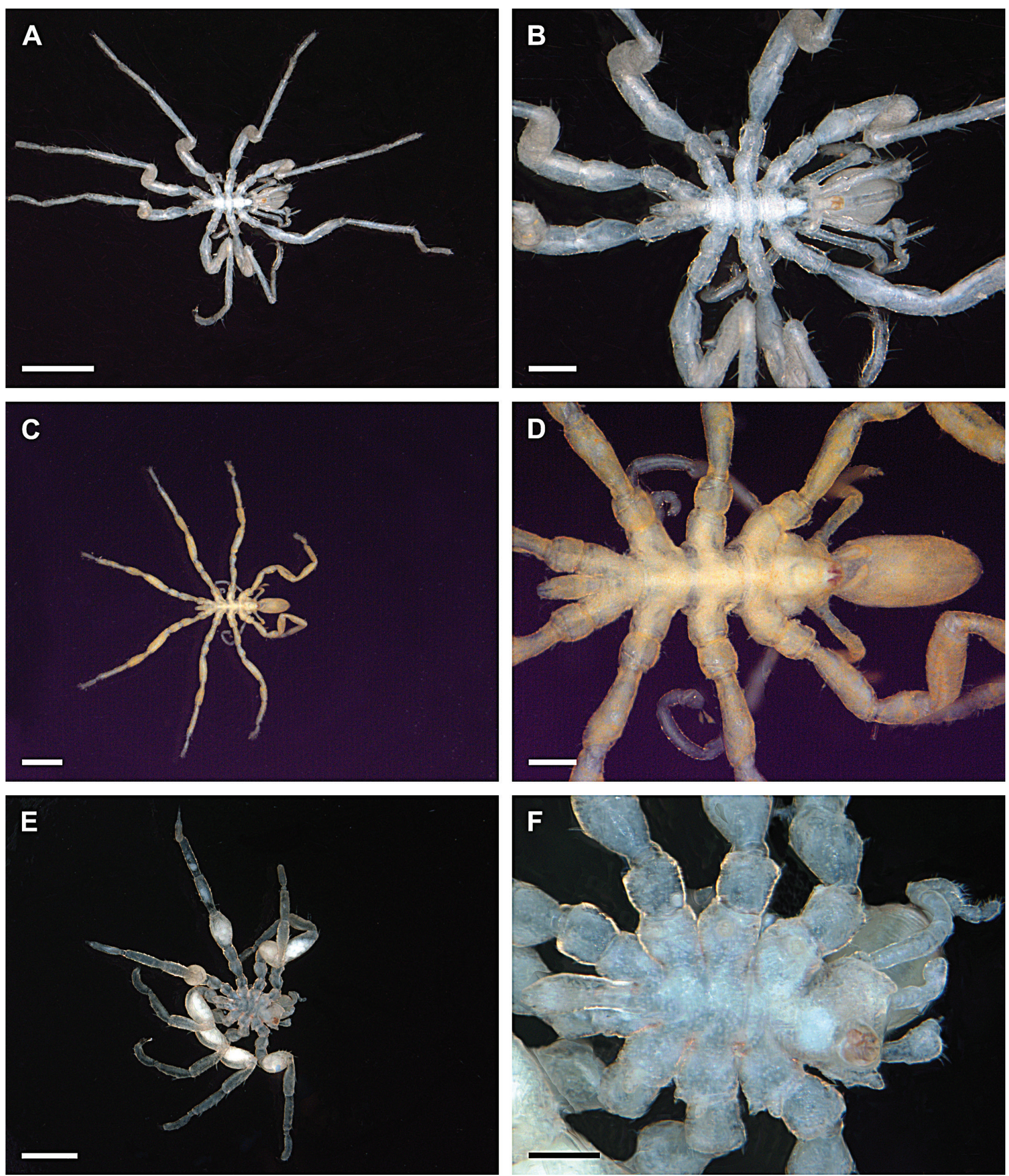

Figure 3. Ammotheidae 2; A, B: Ammothella appendiculata, female, dorsal view; scales $1 \mathrm{~mm}$ and $250 \mu \mathrm{m}$, respectively; C, D: Ammothella biunguiculata, male, dorsal view; scales $1 \mathrm{~mm}$ and $250 \mu \mathrm{m}$, respectively; E, F: Ammothella longipes, female, dorsal view; scales $1 \mathrm{~mm}$ and $250 \mu \mathrm{m}$, respectively. 

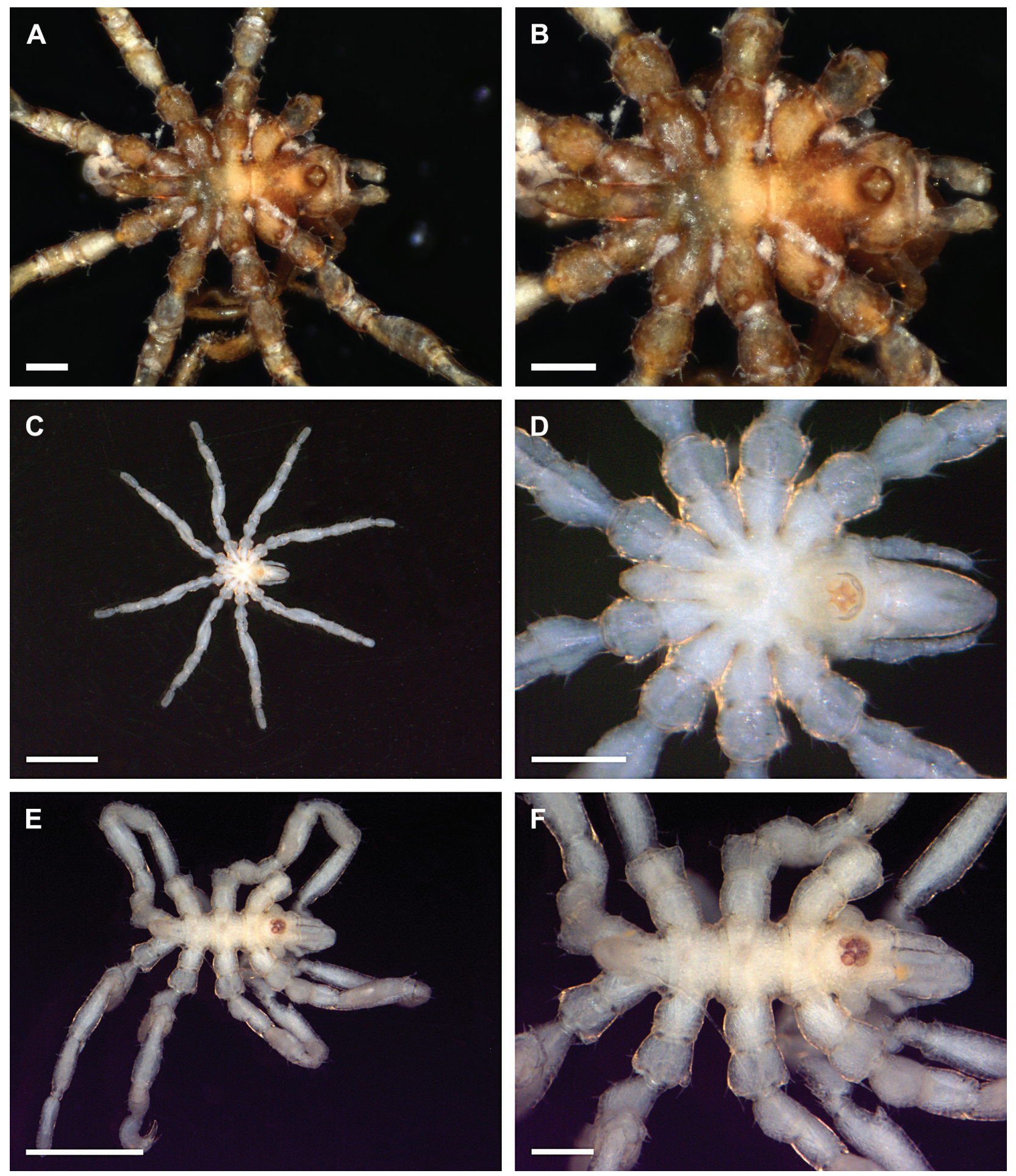

Figure 4. Ammotheidae 3; A, B: Ammothella uniunguiculata, male, dorsal view; scales $200 \mu \mathrm{m}$; C, D: Tanystylum conirostre, male, dorsal view; scales $1 \mathrm{~mm}$ and $250 \mu \mathrm{m}$, respectively; E, F: Trygaeus communis, male, dorsal view; scales $1 \mathrm{~mm}$ and $250 \mu \mathrm{m}$, respectively. 

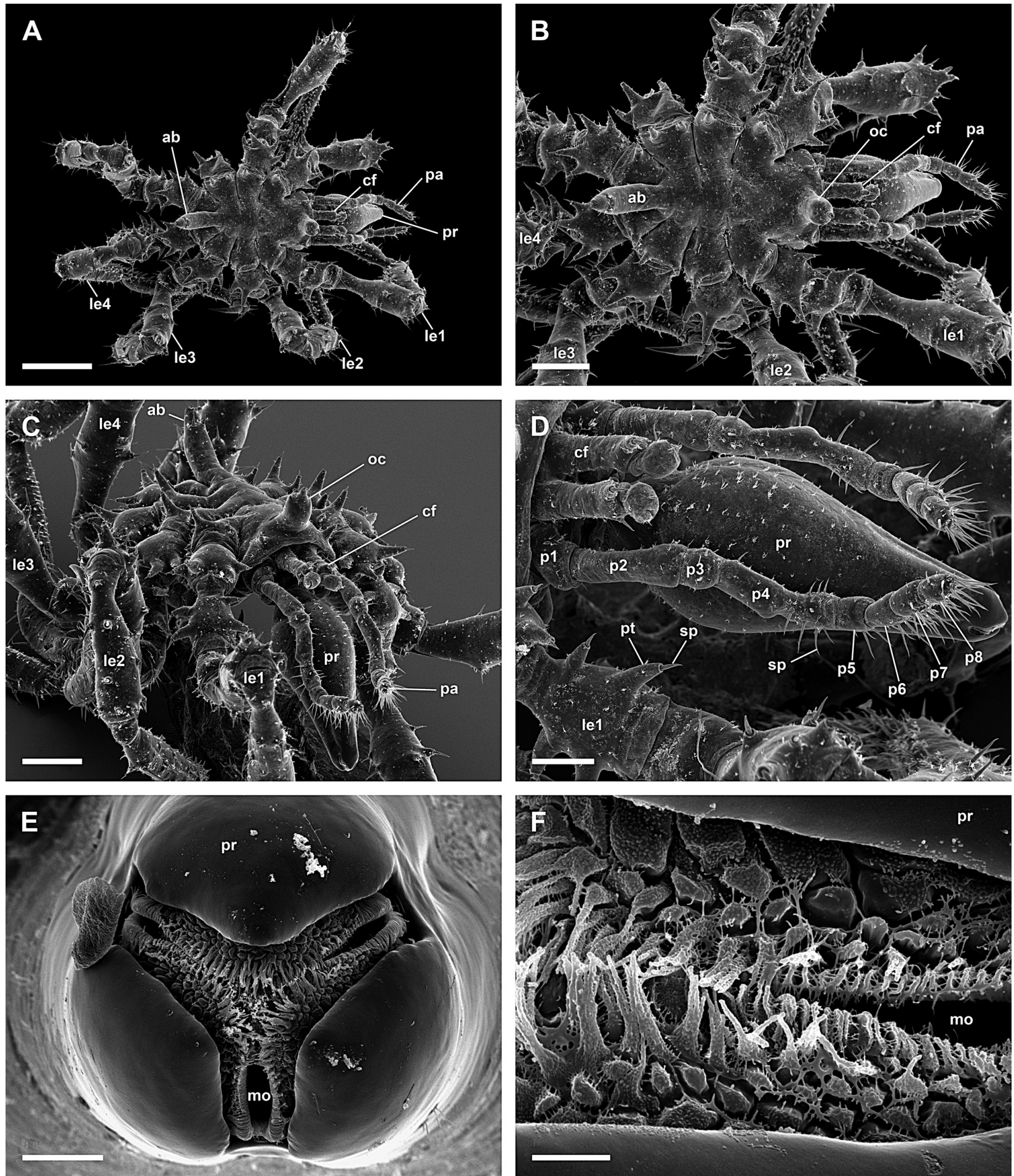

Figure 5. Achelia echinata, male; A: Dorsal view; scale $400 \mu \mathrm{m} ; \mathbf{B}$ : Dorsal view of trunk, lateral processes touch each other; scale $200 \mu \mathrm{m} ; \mathbf{C}$ : Frontal view of trunk, protuberances with spines on lateral process; scale $200 \mu \mathrm{m}$; D: Lateral view of proboscis, palp and chelifores with reduced chela; scale $100 \mu \mathrm{m}$; E: Mouth opening, dorsal is up; scale $20 \mu \mathrm{m}$; F: Mouth opening; scale $5 \mu \mathrm{m}$. 

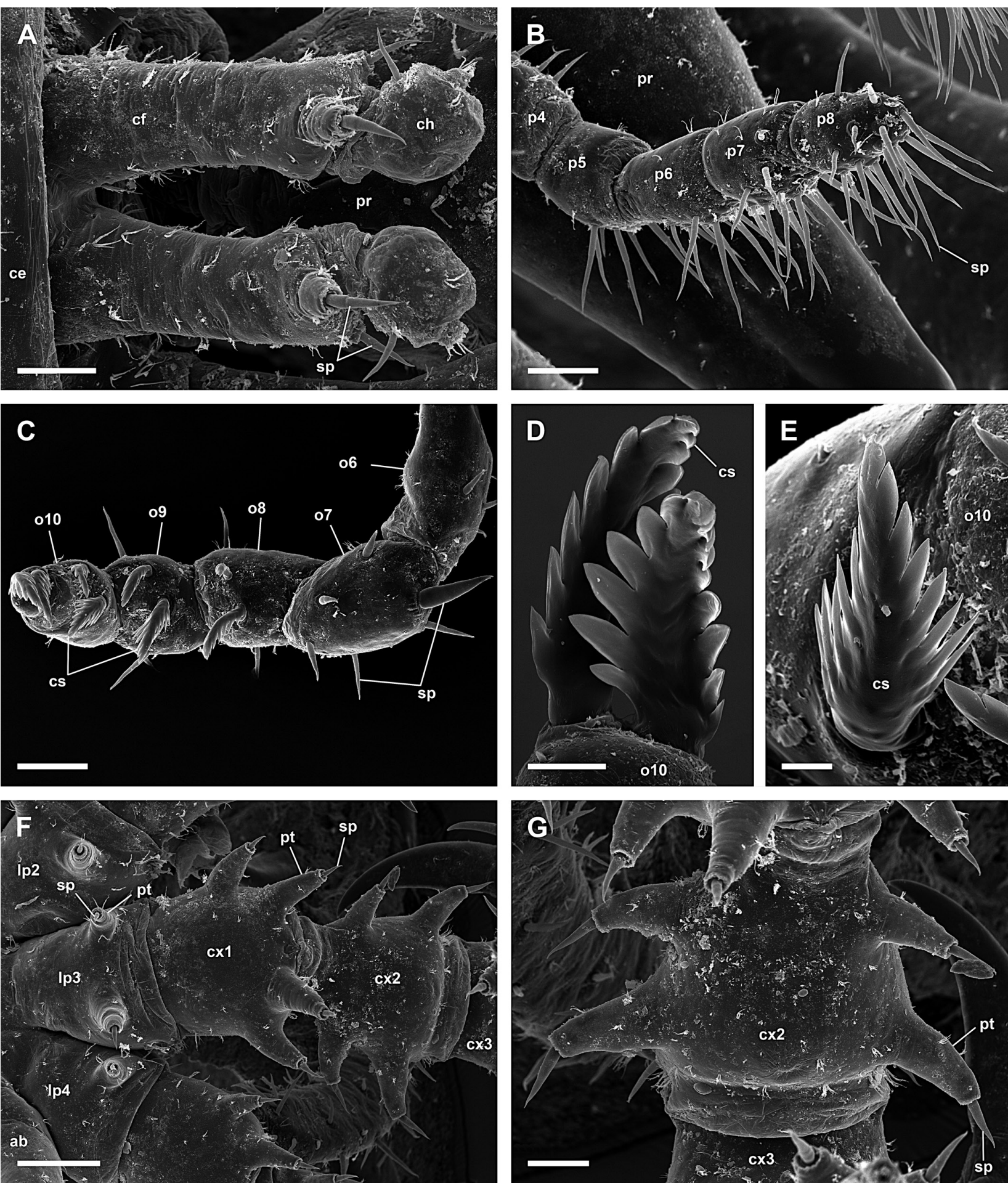

Figure 6. Achelia echinata, male; A: Chelifores with reduced chela; scale $40 \mu \mathrm{m}$; B: Distal articles of right 8-articled palp; scale $40 \mu \mathrm{m} ; \mathbf{C}$ : Distal articles of 10-articled oviger; scale $40 \mu \mathrm{m}$; D, E: Compound spines on last oviger-article; scales $10 \mu \mathrm{m}$ and $5 \mu \mathrm{m}$, respectively; F: Lateral process, coxa 1 and 2 of right 3rd leg, 2 protuberances with spine on each side of coxa 2; scale $100 \mu \mathrm{m}$; G: Coxa 2 with 2 protuberances with spine on each side (right 3rd leg); scale $40 \mu \mathrm{m}$. 

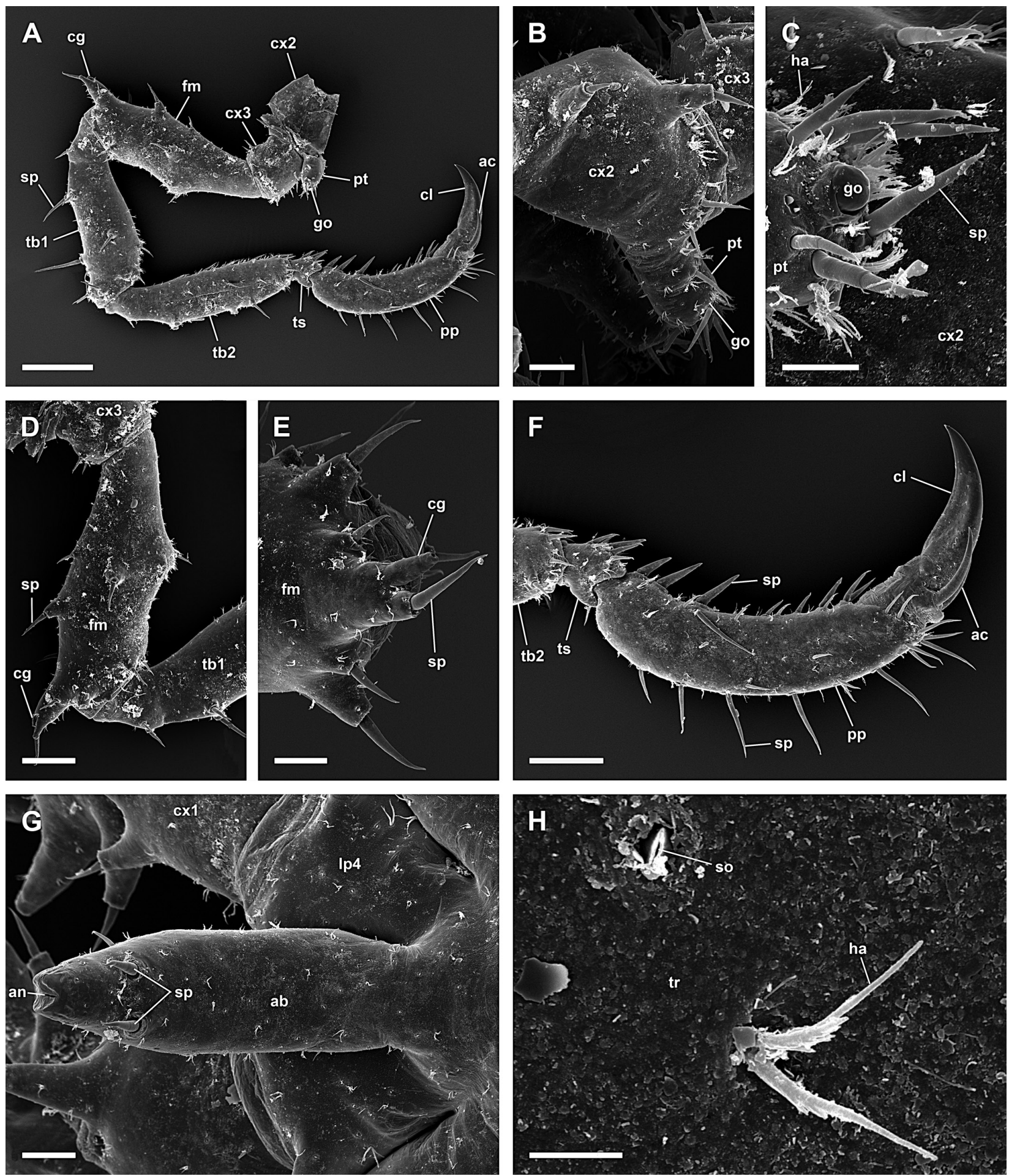

Figure 7. Achelia echinata, male; A: Left 3rd leg; scale $200 \mu \mathrm{m}$; B: Lateral view of coxa 2 with genital protuberance (right 4th leg); scale $40 \mu \mathrm{m}$; C: Genital opening (right 3rd leg); scale $20 \mu \mathrm{m}$; D: Lateral view of femur with cement gland on distal part (left 3rd leg); scale $100 \mu \mathrm{m} ; \mathbf{E}$ : Cement gland (right 4th leg); scale $40 \mu \mathrm{m} ; \mathbf{F}$ : Tarsus, propodus, and claw, auxiliary claws about half as long as claw (left 3rd leg); scale $100 \mu \mathrm{m}$; G: Abdomen; scale $40 \mu \mathrm{m} ; \mathbf{H}$ : Hair and slit organ on dorsal side of trunk; scale $5 \mu \mathrm{m}$. 

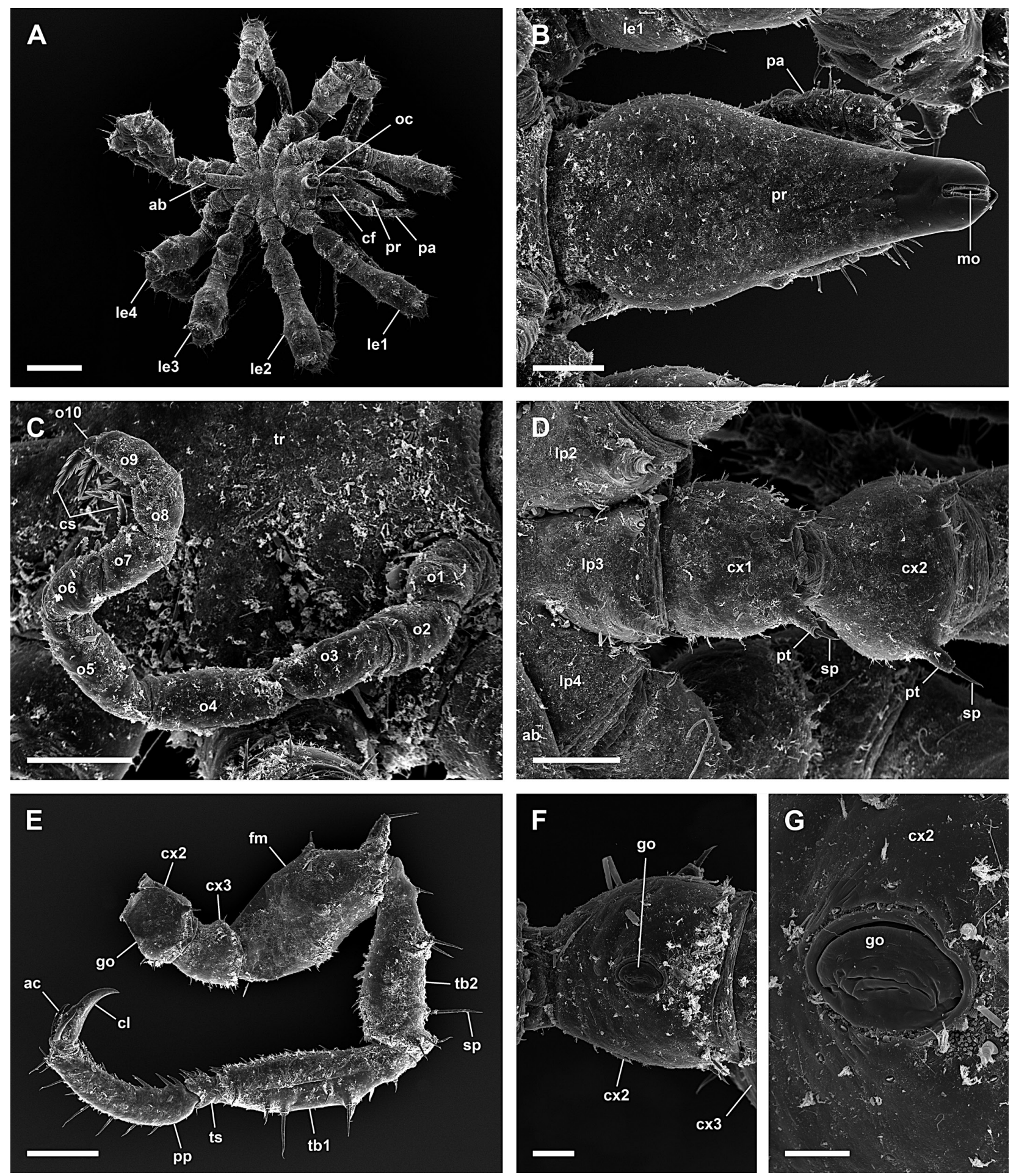

Figure 8. Achelia echinata, female; A: Dorsal view; scale $400 \mu \mathrm{m} ; \mathbf{B}$ : Ventral view of proboscis; scale $100 \mu \mathrm{m}$; C: Left 10-articled oviger; scale $100 \mu \mathrm{m}$; D: Lateral process, coxa 1 and 2 of right 3rd leg, lateral processes touch each other; scale $100 \mu \mathrm{m} ; \mathbf{E}$ : Left 3rd leg; scale $200 \mu \mathrm{m}$; F: Ventral view of coxa 2 with genital opening, distal is right (left 3rd leg); scale $40 \mu \mathrm{m}$; G: Genital opening; scale $20 \mu \mathrm{m}$. 

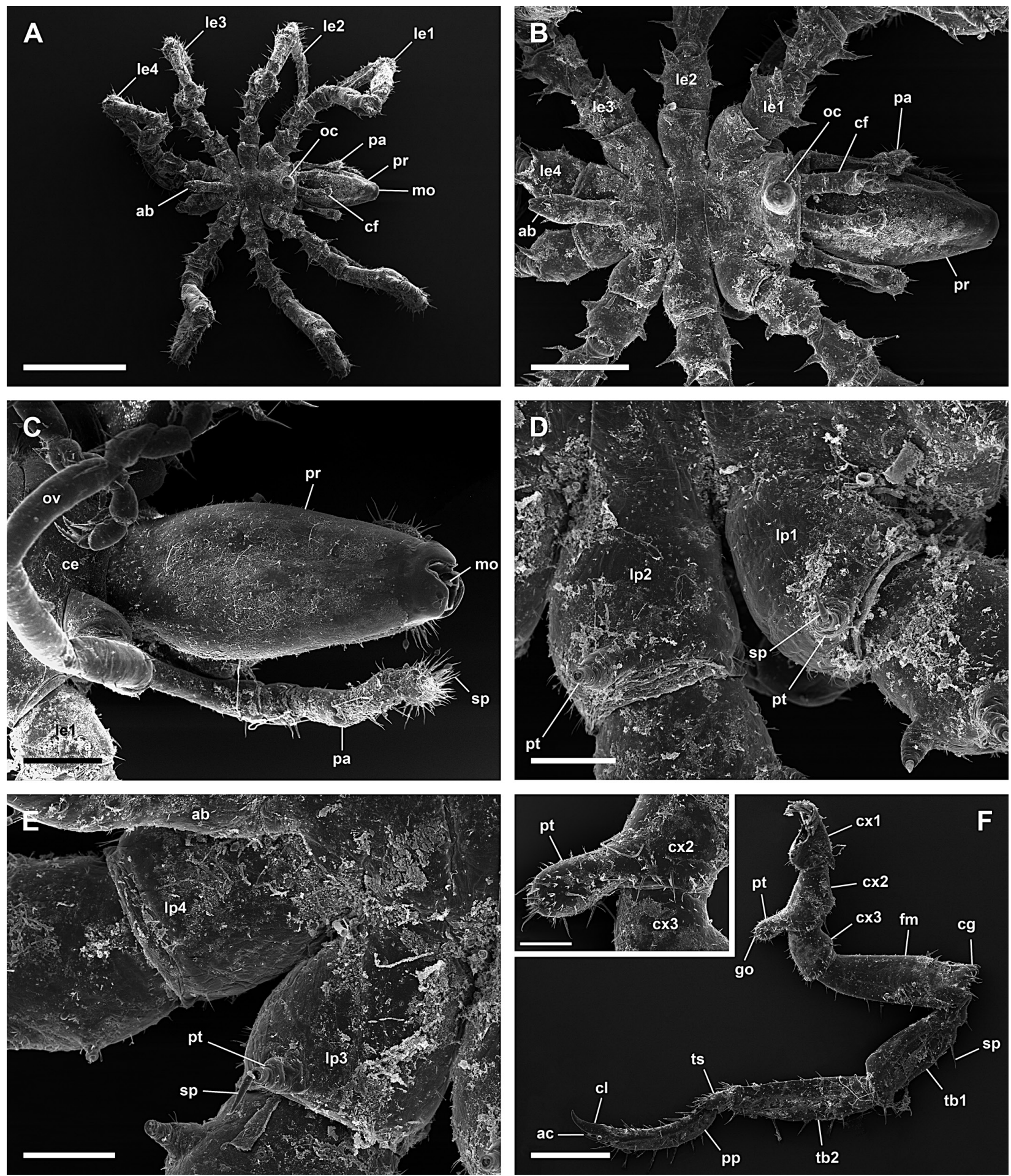

Figure 9. Achelia langi, male; A: Dorsal view; scale $1 \mathrm{~mm}$; B: Dorsal view of trunk; scale $400 \mu \mathrm{m}$; C: Ventral view of proboscis; scale $200 \mu \mathrm{m}$; D: Dorsal view of right lateral processes 1 and 2, 2 protuberances with spine on lateral process 1 and 1 on lateral process 2; scale $100 \mu \mathrm{m}$; E: Dorsal view of right lateral processes 3 and 4, 1 protuberance with spine on lateral process 3 and lateral process 4 without protuberance or spine; scale $100 \mu \mathrm{m}$; F: Left 3rd leg; scale $400 \mu \mathrm{m}$; insert: Genital protuberance; scale $100 \mu \mathrm{m}$. 

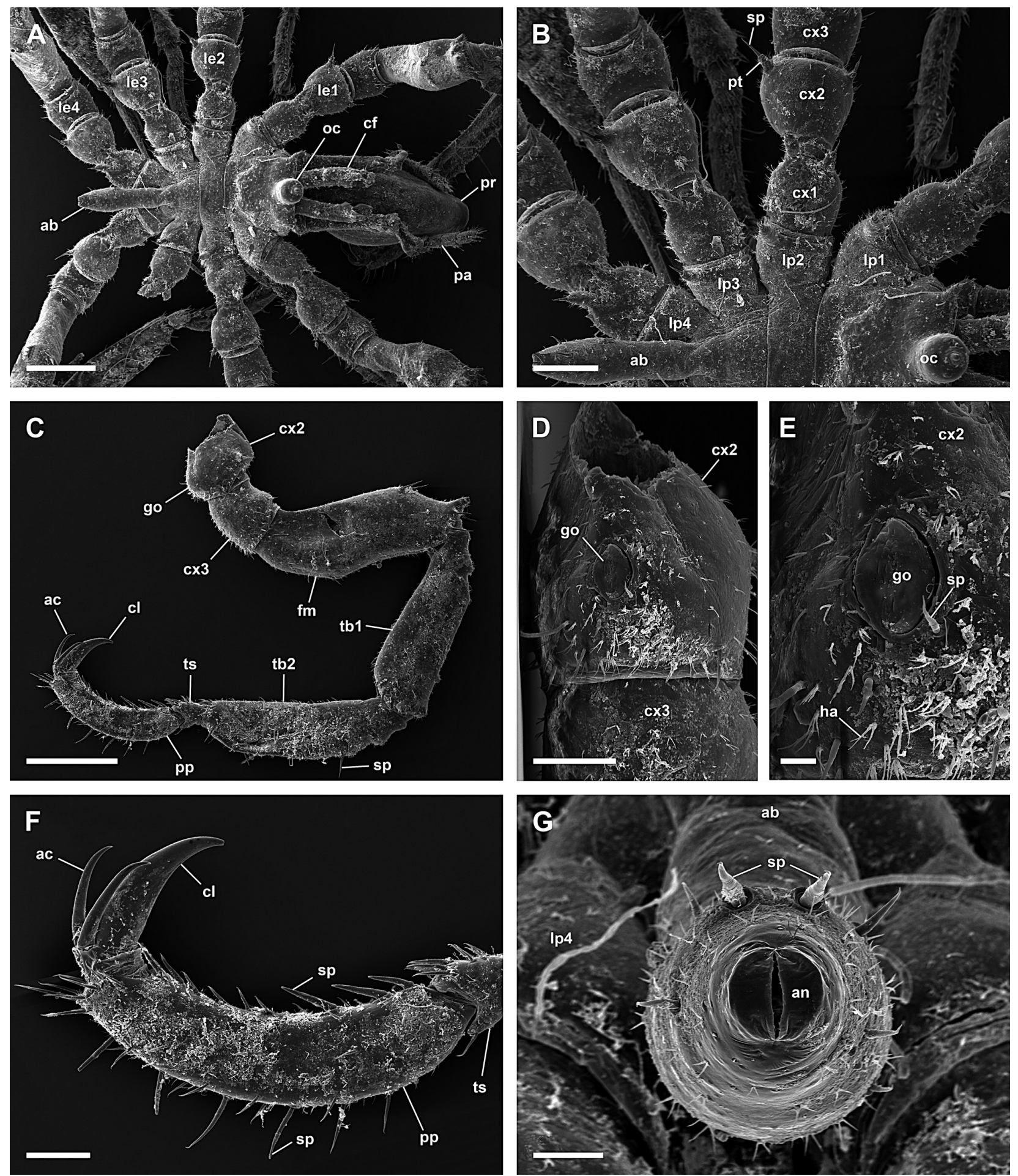

Figure 10. Achelia langi, female; A: Dorsal view of trunk; scale $400 \mu \mathrm{m} ; \mathbf{B}$ : Dorsal view of left lateral processes, 1 protuberance with spine on lateral process 1-3, lateral process 4 without protuberance or spine; scale $200 \mu \mathrm{m}$; C: Right 3rd leg; scale $400 \mu \mathrm{m}$; D: Ventral view of coxa 2 with genital opening, distal is down (right 3rd leg); scale $100 \mu \mathrm{m}$; E: Genital opening; scale $20 \mu \mathrm{m}$; F: Tarsus, propodus, and claw, auxiliary claws about $2 / 3$ of length of claw; scale $100 \mu \mathrm{m}$; G: Abdomen with anus; scale $40 \mu \mathrm{m}$. 

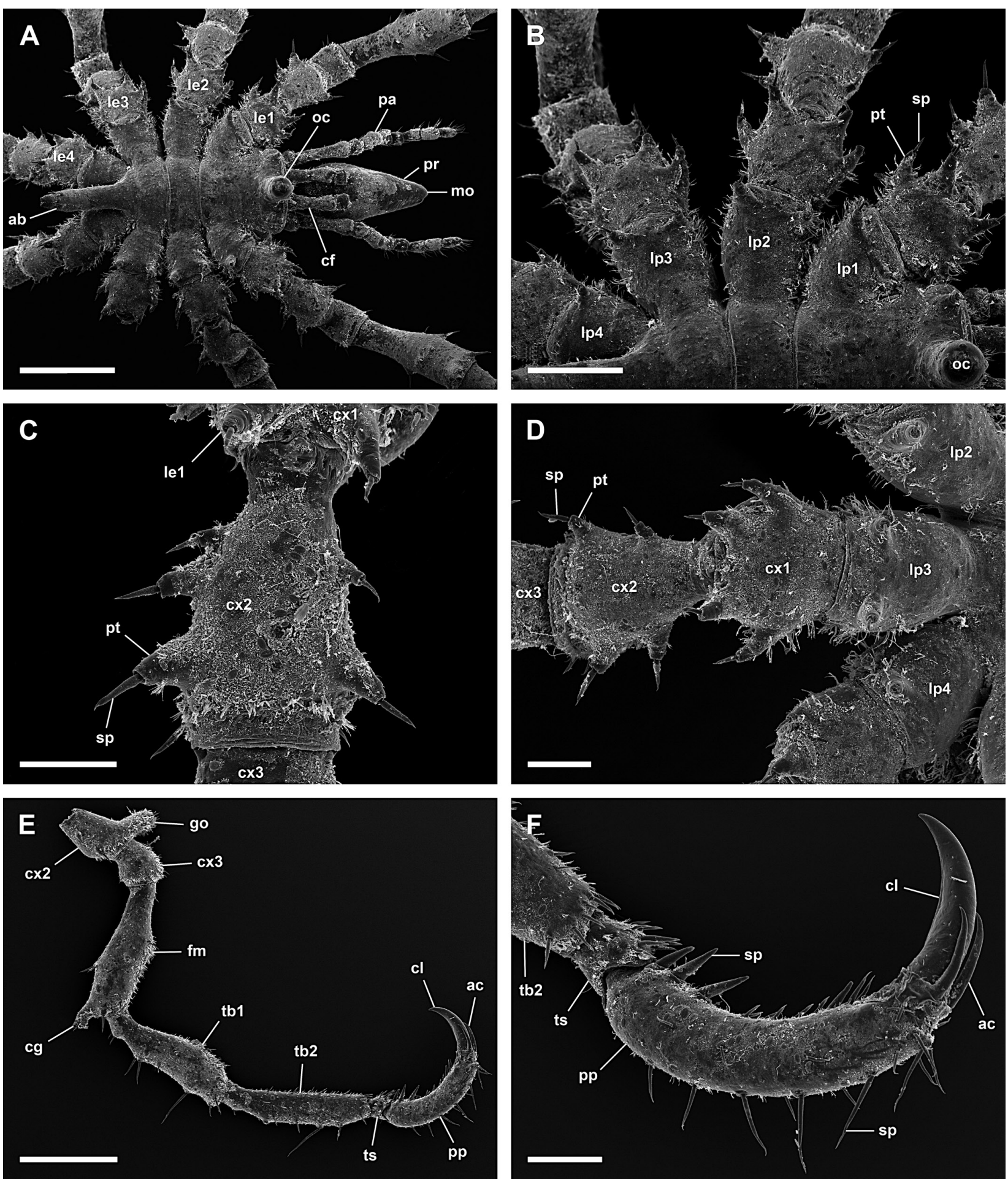

Figure 11. Achelia vulgaris, male; A: Dorsal view of trunk; scale $400 \mu \mathrm{m} ; \mathbf{B}$ : Dorsal view of left lateral processes, lateral processes do not touch each other; scale $200 \mu \mathrm{m} ; \mathbf{C}: 2$ protuberances with spine on right side and 3 on left side of coxa 2, distal is down (right 1st leg); scale $100 \mu \mathrm{m}$; D: Lateral process, coxa 1 and 2, 2 protuberances with spine on each side of coxa 2 (left 3rd leg); scale $100 \mu \mathrm{m}$; E: Right 3rd leg; scale $400 \mu \mathrm{m} ; \mathbf{F}$ : Tarsus, propodus, and claw, auxiliary claws about half as long as claw; scale $100 \mu \mathrm{m}$. 

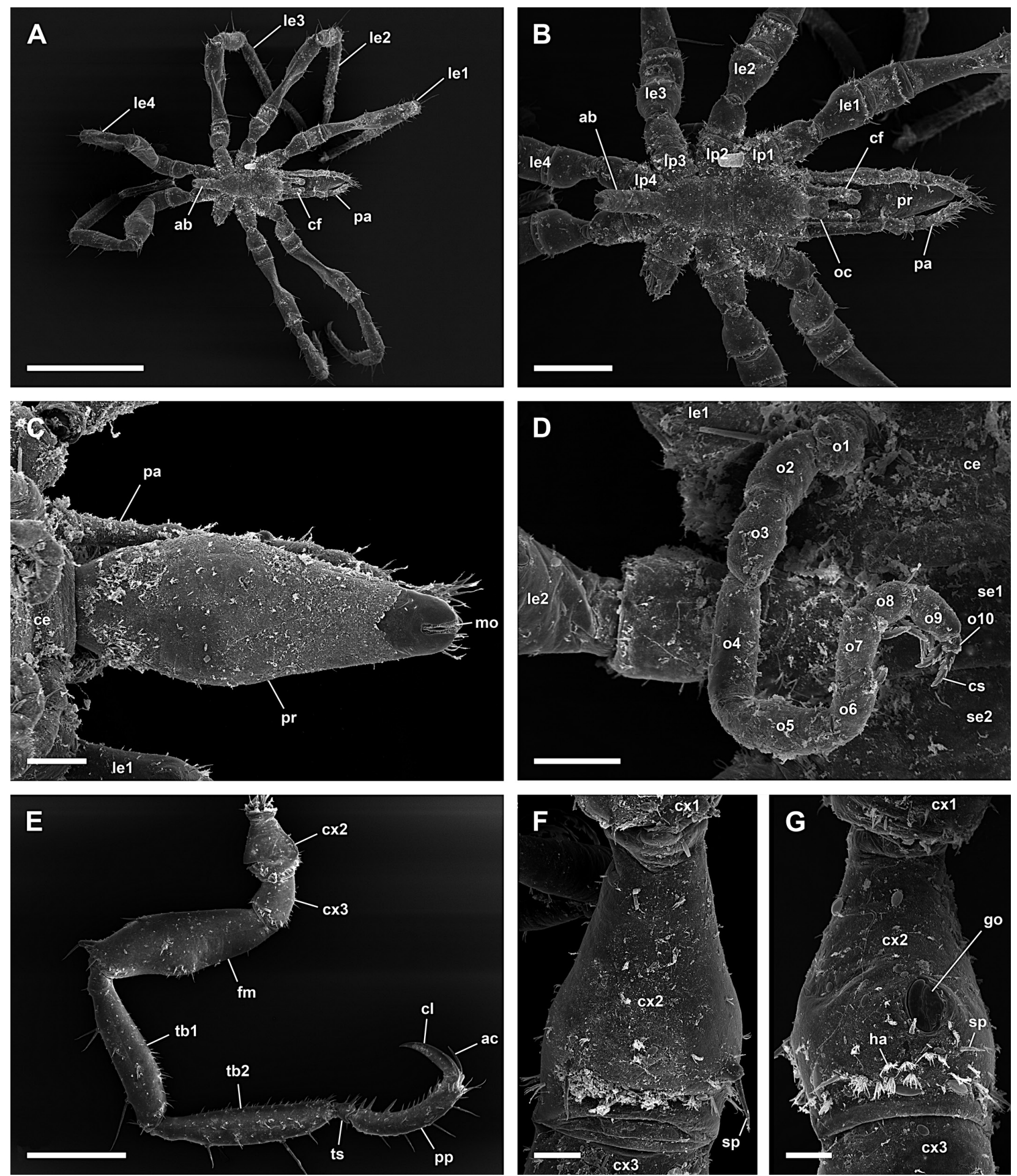

Figure 12. Achelia vulgaris, female; A: Dorsal view; scale $1 \mathrm{~mm}$; B: Dorsal view of trunk, lateral processes do not touch each other; scale $300 \mu \mathrm{m}$; C: Ventral view of proboscis; scale $100 \mu \mathrm{m}$; D: Right 10-articled oviger; scale $100 \mu \mathrm{m}$; E: Right 3rd leg; scale $400 \mu \mathrm{m}$; F: Dorsal view of coxa 2 (left 3rd leg); scale $40 \mu \mathrm{m}$; G: Ventral view of coxa 2 with genital opening (left 3rd leg); scale $40 \mu \mathrm{m}$. 

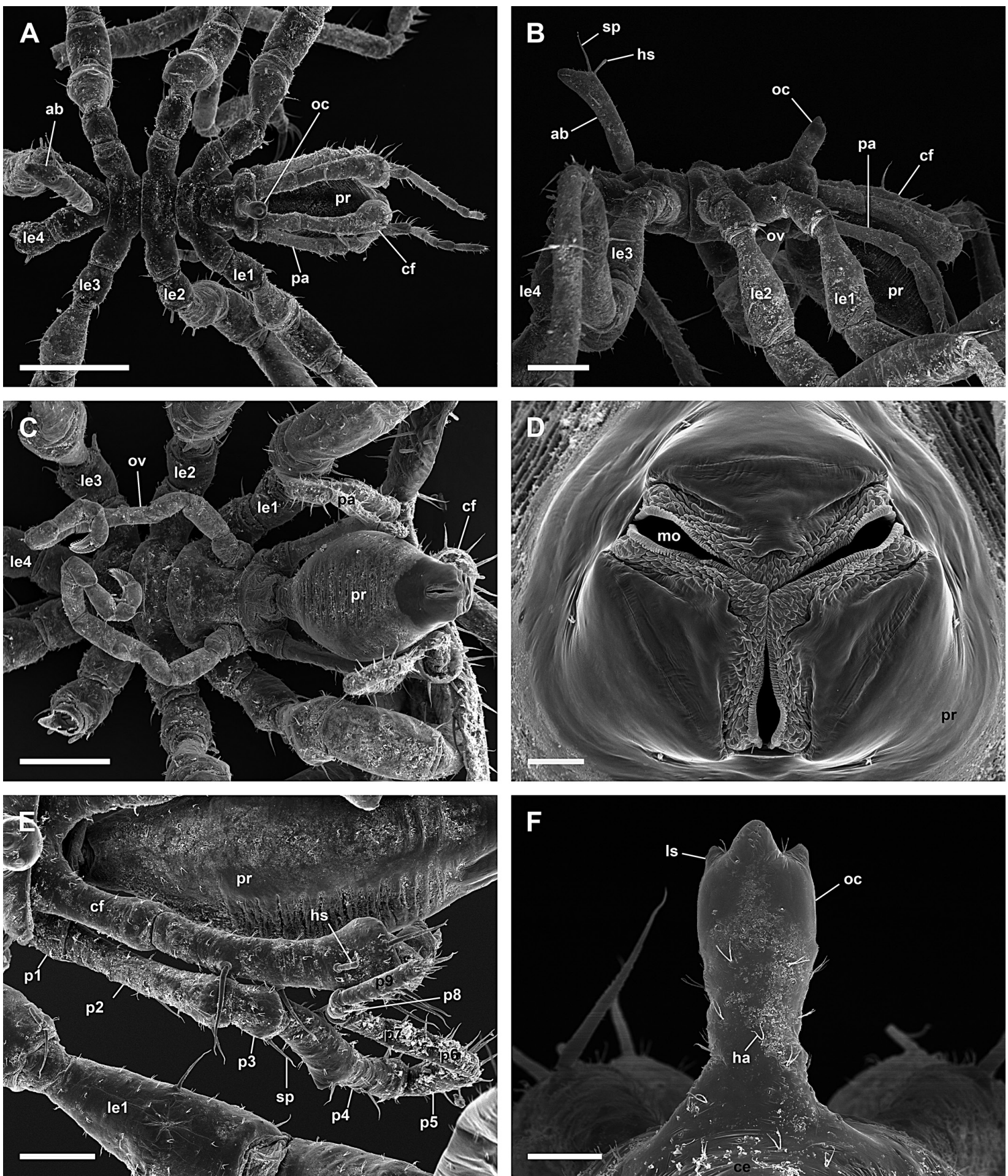

Figure 13. Ammothella appendiculata, female; A: Dorsal view of trunk; scale $400 \mu \mathrm{m}$; B: Lateral view of trunk; scale $200 \mu \mathrm{m}$; C: Ventral view of trunk; scale $200 \mu \mathrm{m}$; D: Mouth opening, dorsal is up; scale $20 \mu \mathrm{m}$; E: Right chelifore with reduced chela and right 9-articled palp; scale $100 \mu \mathrm{m}$; F: Ocular tubercle with lateral sense organ; scale $40 \mu \mathrm{m}$. 

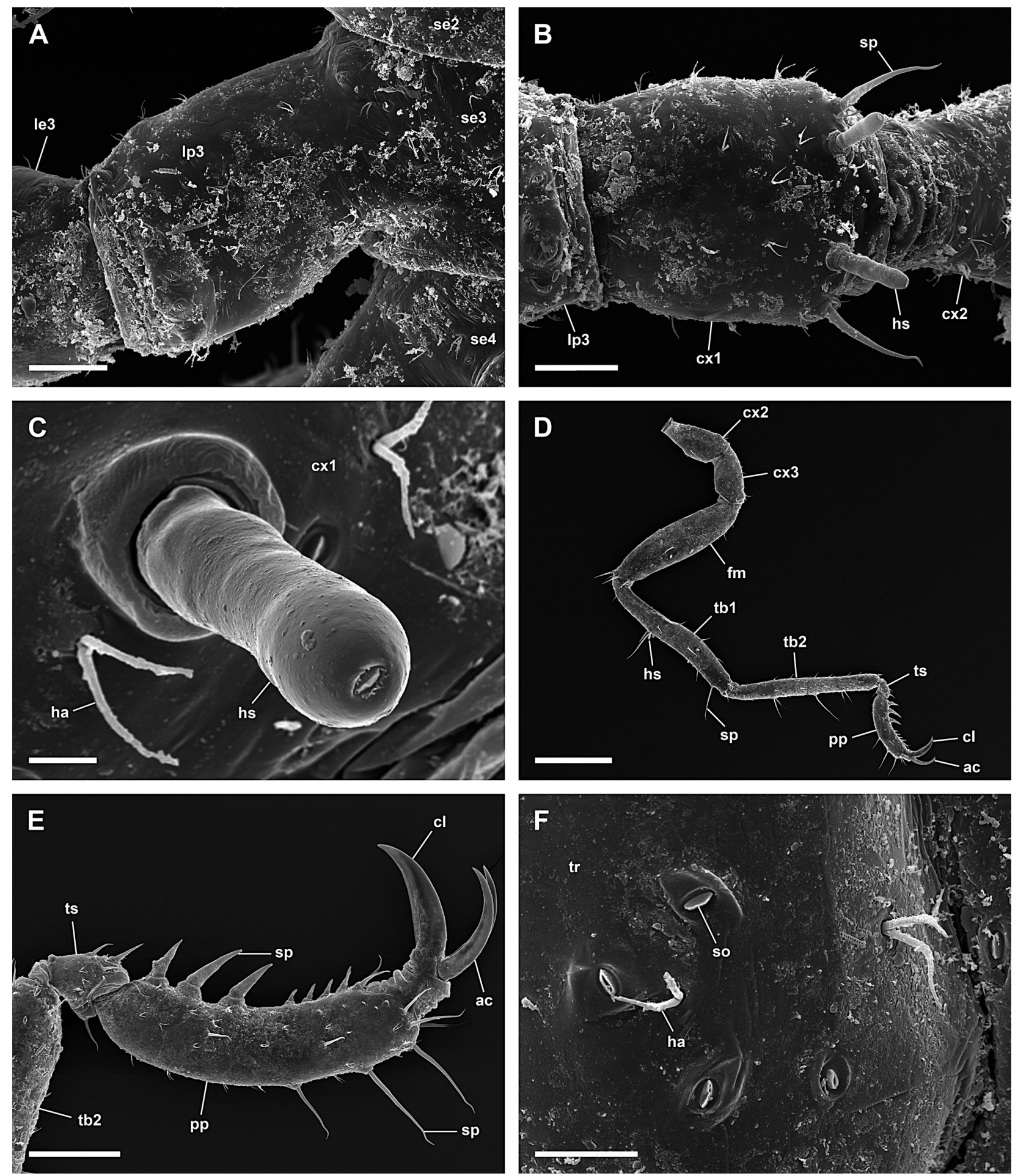

Figure 14. Ammothella appendiculata, female; A: Lateral process of left 3rd leg; scale $40 \mu \mathrm{m}$; B: Coxa 1 of right 3rd leg with hollow spines; scale $40 \mu \mathrm{m}$; C: Hollow spine; scale $5 \mu \mathrm{m}$; D: Right 4th leg; scale $400 \mu \mathrm{m}$; E: Tarsus, propodus, and claws (right 4th leg); scale $100 \mu \mathrm{m}$; F: Hairs and slit organs of dorsal side of trunk; scale $10 \mu \mathrm{m}$. 

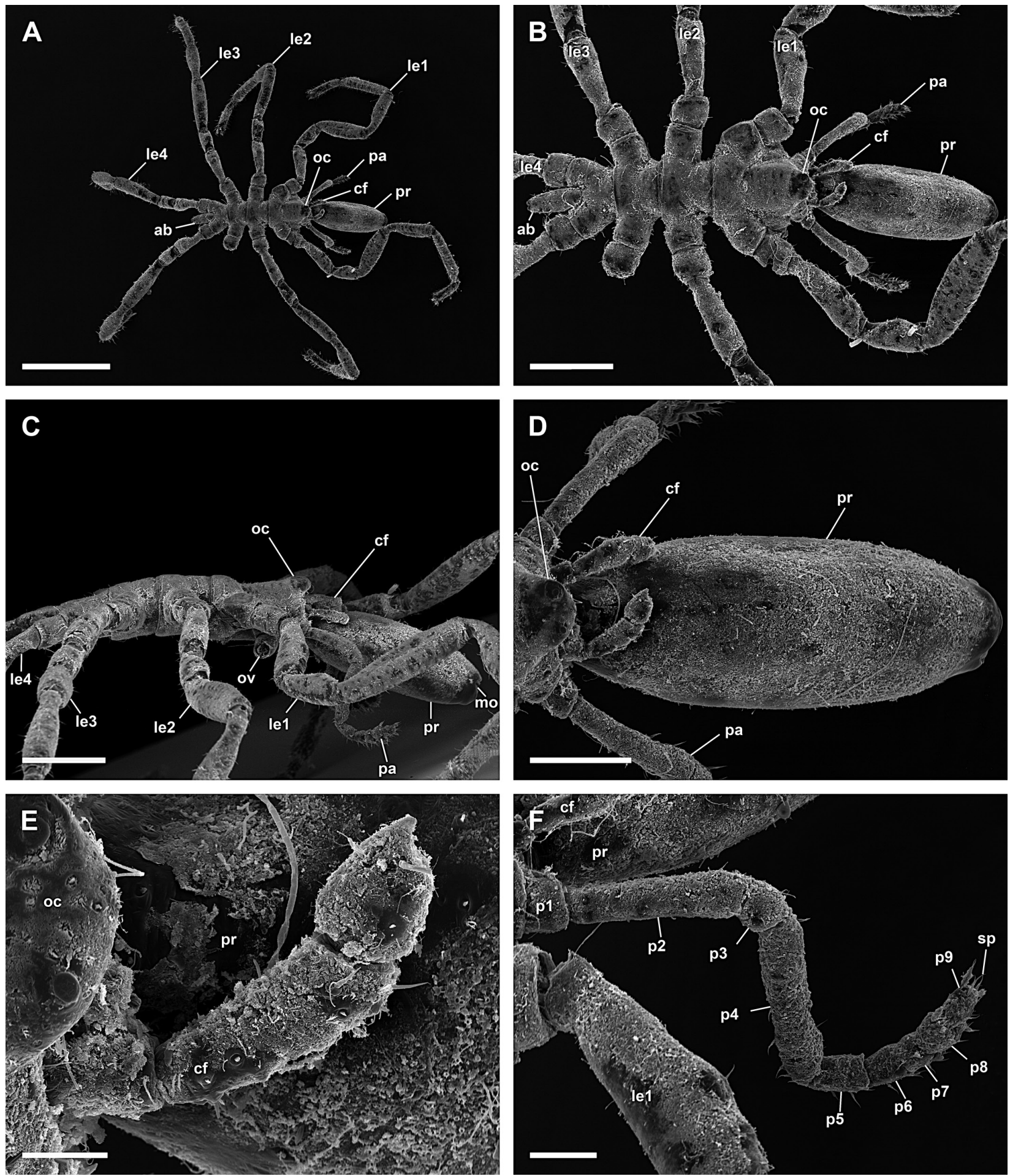

Figure 15. Ammothella biunguiculata, male; A: Dorsal view; scale $1 \mathrm{~mm}$; B: Dorsal view of trunk; scale $400 \mu \mathrm{m}$; C: Lateral view of trunk, oviger dissected; scale $400 \mu \mathrm{m}$; D: Dorsal view of proboscis; scale $100 \mu \mathrm{m}$; E: Right chelifore with reduced chela; scale $40 \mu \mathrm{m}$; F: Right 9-articled palp; scale $100 \mu \mathrm{m}$. 

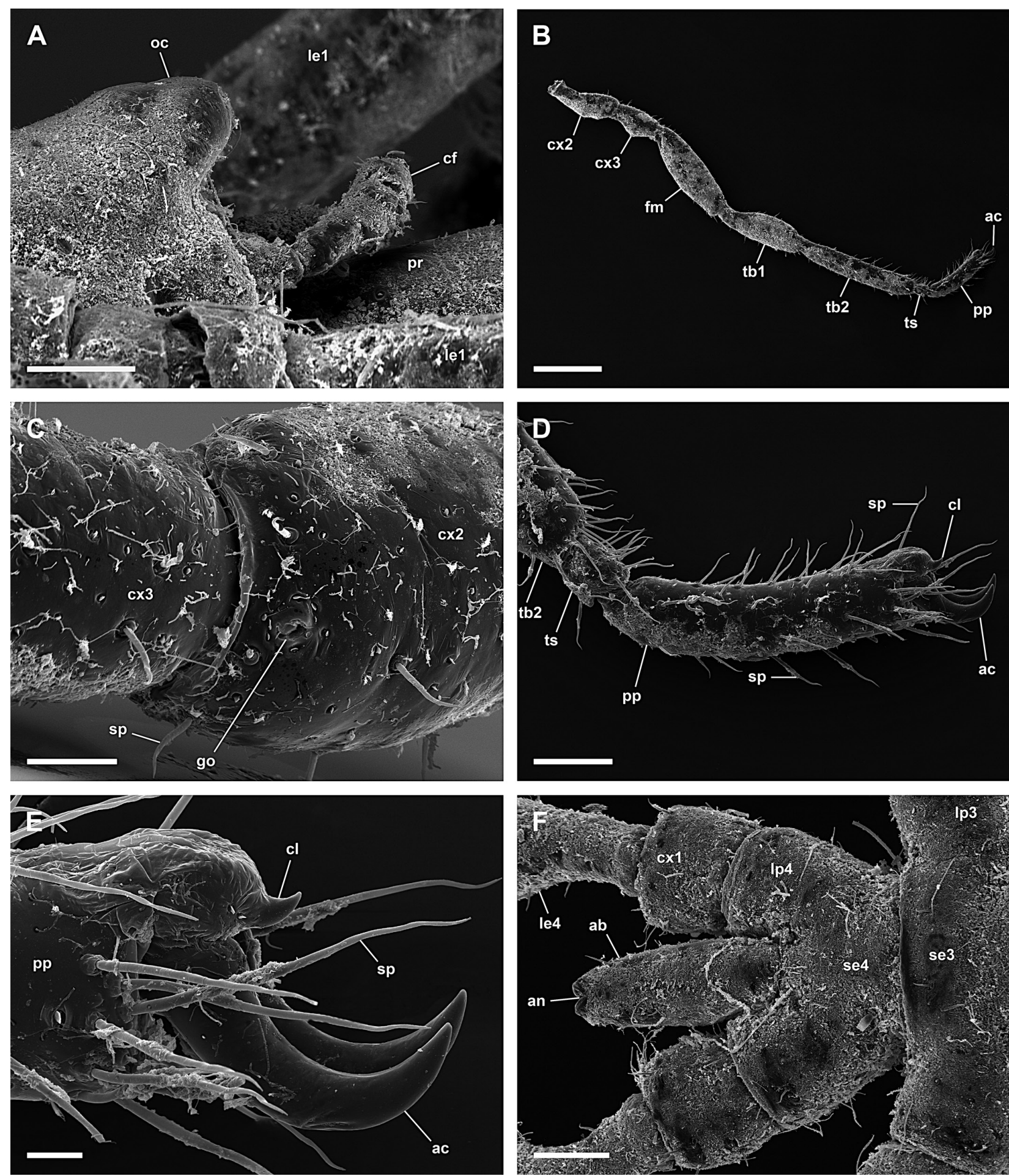

Figure 16. Ammothella biunguiculata, male; A: Lateral view of ocular tubercle; scale $100 \mu \mathrm{m}$; B: Right 3rd leg; scale $400 \mu \mathrm{m}$; C: Ventral view of coxa 2 with genital opening, distal is left (right $3 \mathrm{rd} \mathrm{leg}$ ); scale $40 \mu \mathrm{m}$; D: Tarsus and propodus with reduced claw and dominating auxiliary claws; scale $100 \mu \mathrm{m} ; \mathbf{E}$ : Reduced claw and dominating auxiliary claws; scale $20 \mu \mathrm{m} ; \mathbf{F}$ : Dorsal view of trunk segment 4 and abdomen; scale $100 \mu \mathrm{m}$. 

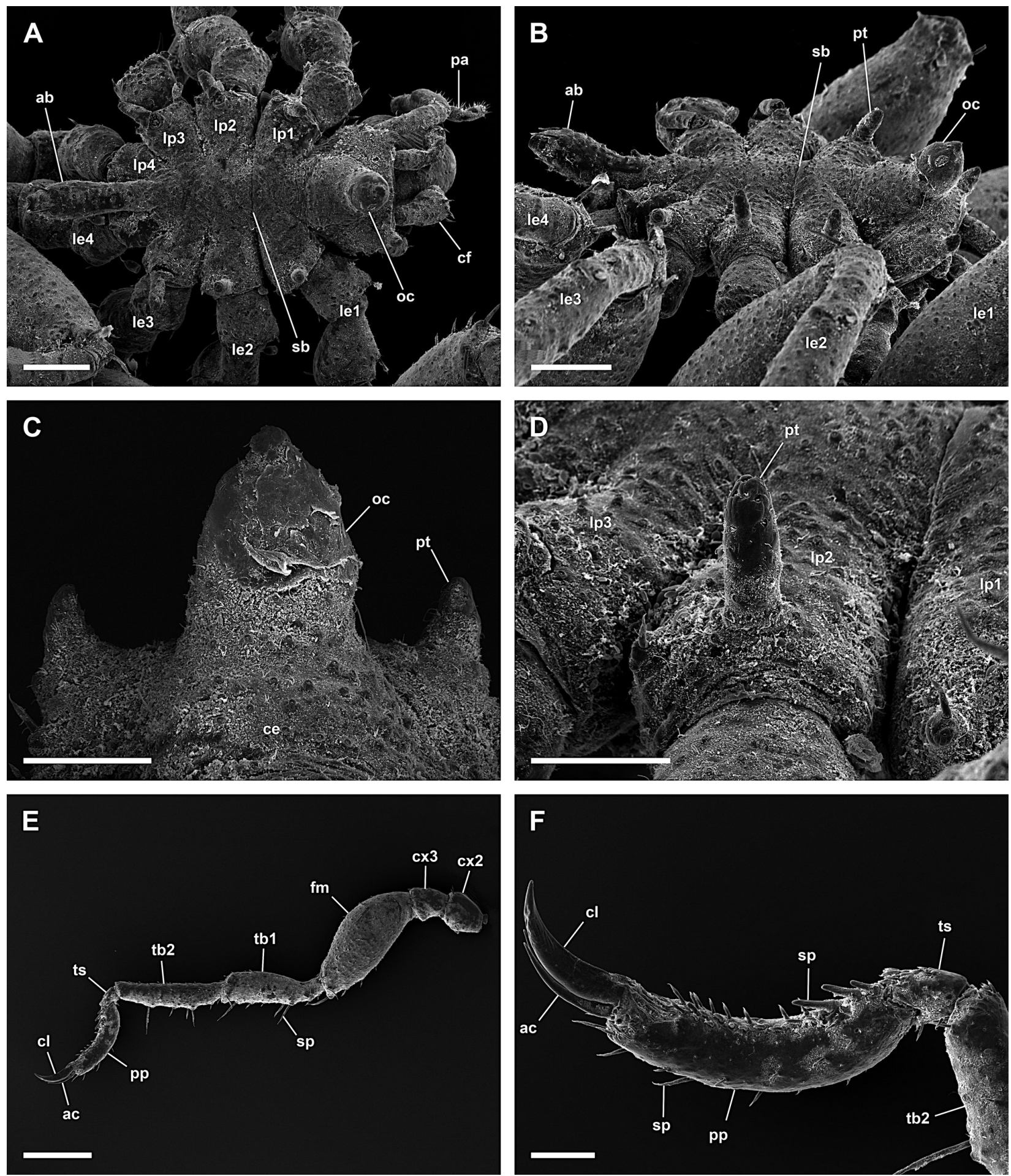

$\mathbf{F}$

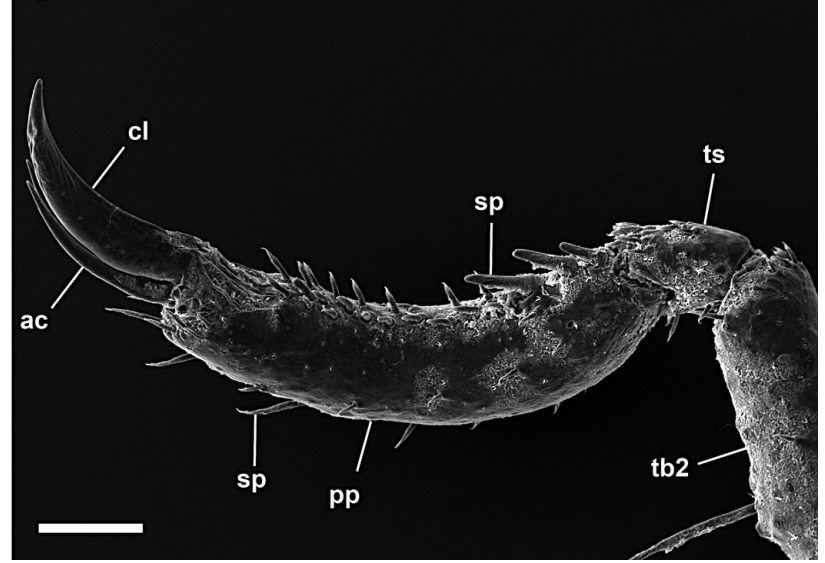

Figure 17. Ammothella longipes, female; A: Dorsal view of trunk; scale $200 \mu \mathrm{m}$; B: Lateral view of trunk, one protuberance without spine aside on lateral processes; scale $200 \mu \mathrm{m}$; C: Ocular tubercle; scale $100 \mu \mathrm{m}$; D: Right lateral process; scale $100 \mu \mathrm{m}$; E: Left 3rd leg; scale $400 \mu \mathrm{m} ; \mathbf{F}$ : Tarsus, propodus, and claws (left 3rd leg); scale $100 \mu \mathrm{m}$. 

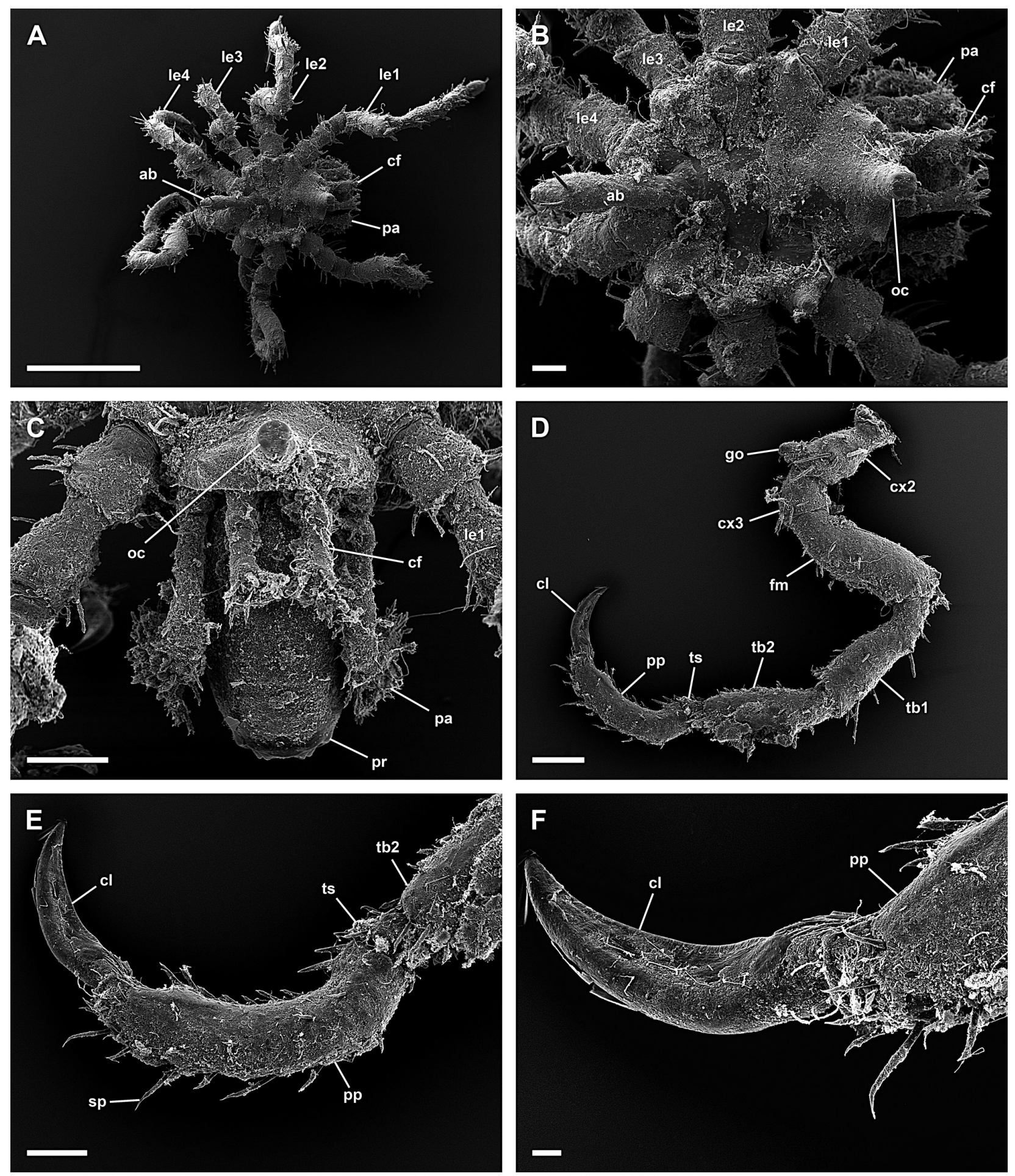

Figure 18. Ammothella uniunguiculata, male; A: Dorsal view; scale $1 \mathrm{~mm}$; B: Dorsal view of trunk; scale $100 \mu \mathrm{m}$; C: Cephalon with proboscis, chelifore, and palps; scale $200 \mu \mathrm{m}$; D: Right 3rd leg; scale $200 \mu \mathrm{m}$; E: Tibia 2, tarsus, and propodus with claw (right 3rd leg); scale $100 \mu \mathrm{m} ; \mathbf{F}$ : Propodus with claw; scale $20 \mu \mathrm{m}$. 

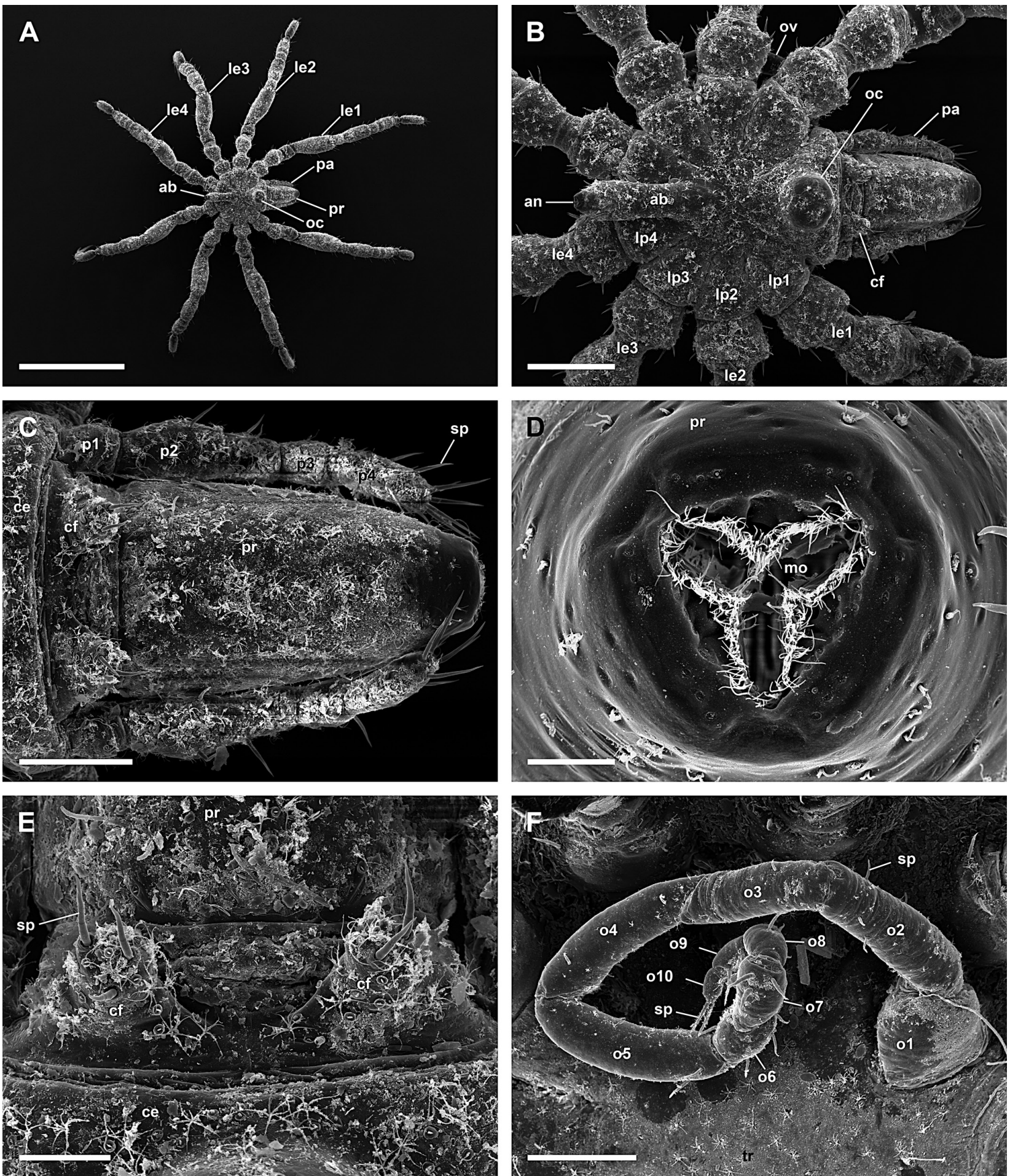

Figure 19. Tanystylum conirostre, male; A: Dorsal view; scale $1 \mathrm{~mm}$; B: Dorsal view of trunk; scale $200 \mu \mathrm{m}$; C: Dorsal view of proboscis and 4-articled palp; scale $100 \mu \mathrm{m}$; D: Mouth opening; scale $20 \mu \mathrm{m}$; E: Reduced chelifores, frontal is up; scale $40 \mu \mathrm{m}$; F: Right 10-articled oviger; scale $100 \mu \mathrm{m}$. 

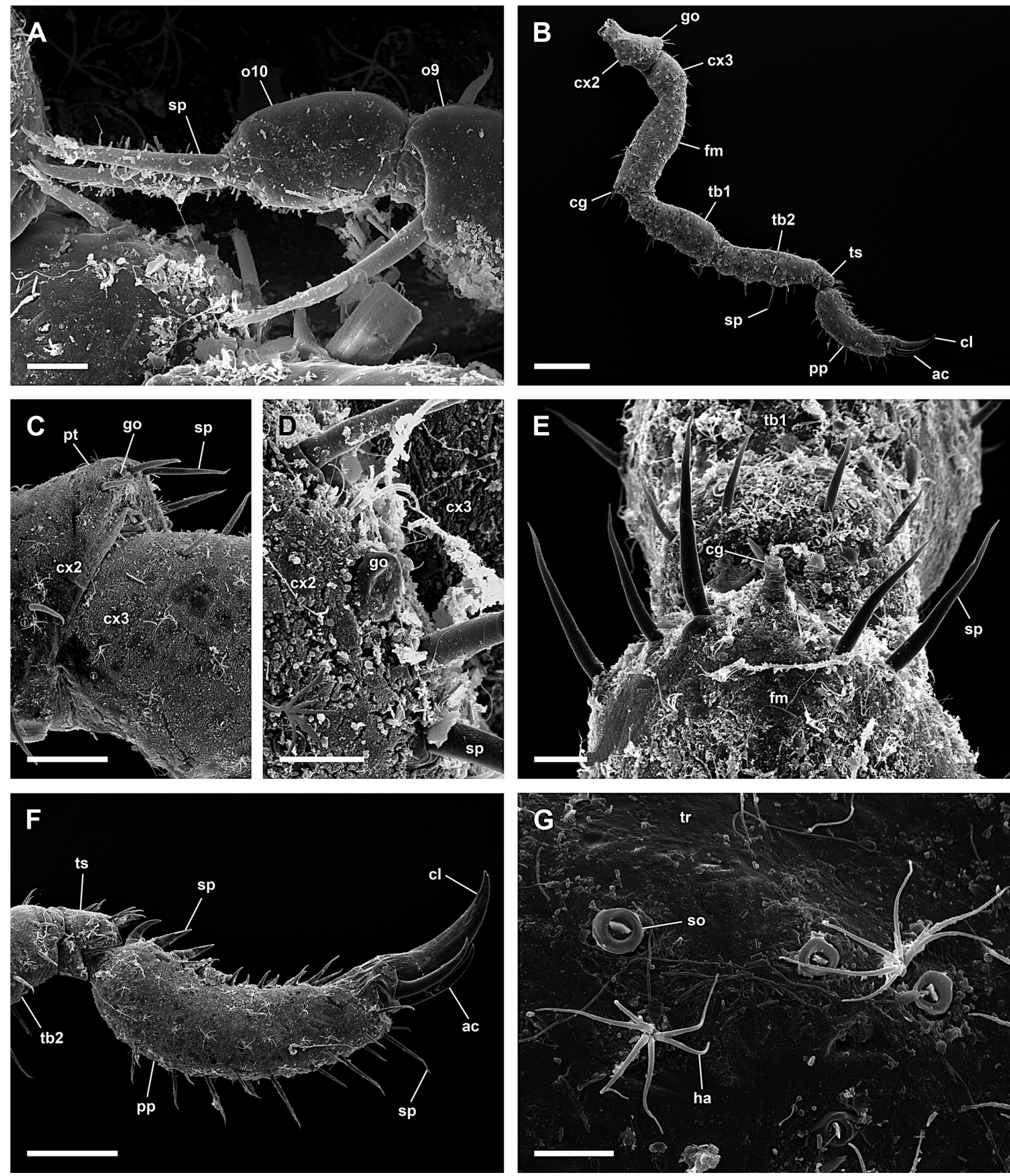

Figure 20. Tanystylum conirostre, male; A: Distal articles of right oviger with spines on tip; scale $10 \mu \mathrm{m}$; B: Left 3rd leg; scale $100 \mu \mathrm{m}$; C: Lateral view of coxa 2 with genital opening on a protuberance, distal is right (left 4th leg); scale $40 \mu \mathrm{m}$; D: Genital opening; scale $10 \mu \mathrm{m} ; \mathbf{E}$ : Cement gland on femur, distal is up (left 3rd leg); scale $20 \mu \mathrm{m} ; \mathbf{F}$ : Tarsus, propodus, and claws (left 3rd leg); scale $100 \mu \mathrm{m}$; G: Hairs and slit organs on dorsal side of trunk; scale $10 \mu \mathrm{m}$. 

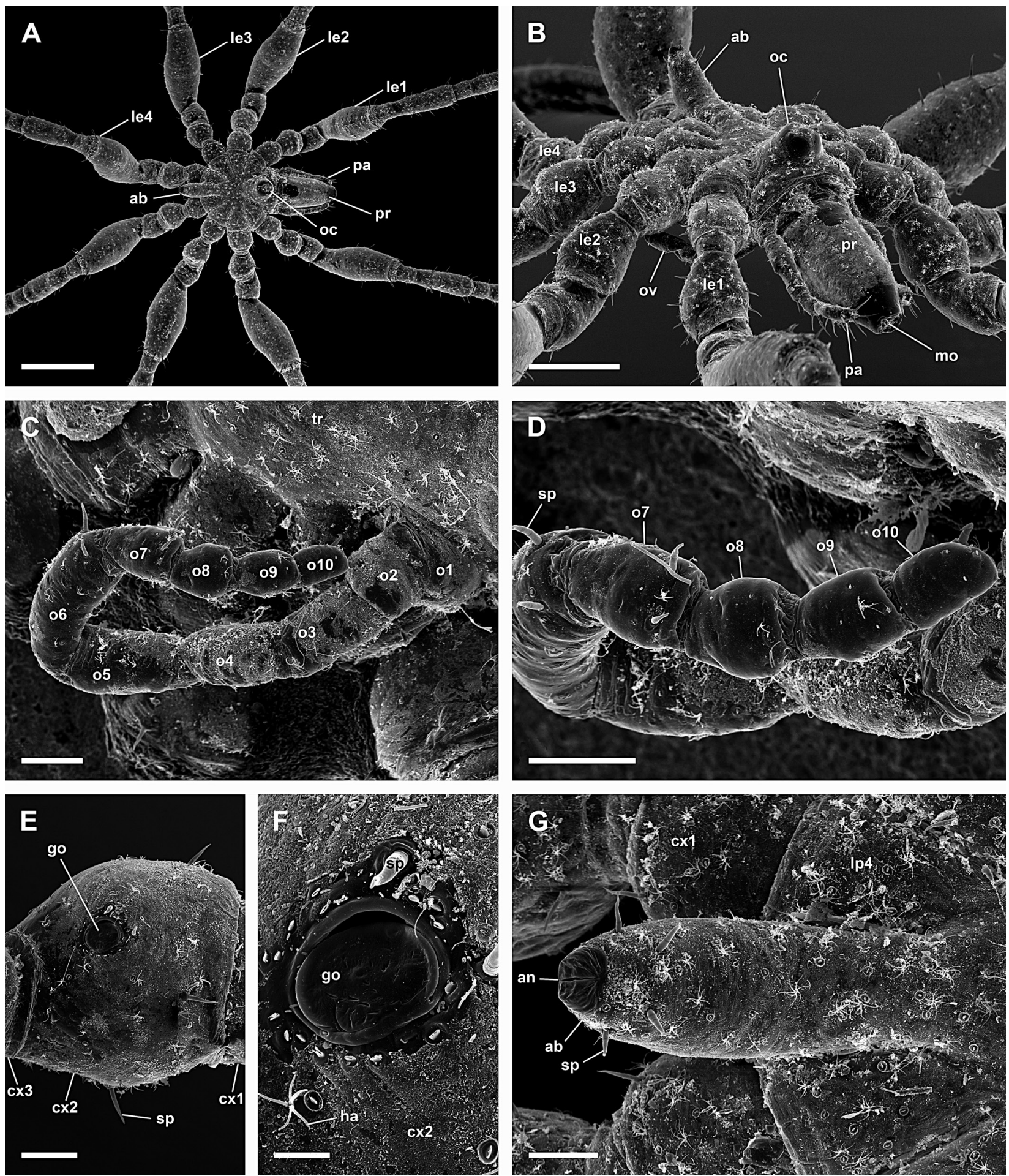

Figure 21. Tanystylum conirostre, female; A: Dorsal view of trunk; scale $400 \mu \mathrm{m}$; B: Frontal view of trunk; scale $200 \mu \mathrm{m}$; C: Left 10-articled oviger; scale $40 \mu \mathrm{m}$; D: Distal articles of left oviger; scale $40 \mu \mathrm{m} ; \mathbf{E}$ : Ventral view of coxa 2 with genital opening, distal is right (right 4th leg); scale $40 \mu \mathrm{m}$; F: Genital opening; scale $10 \mu \mathrm{m}$; G: Abdomen; scale $40 \mu \mathrm{m}$. 

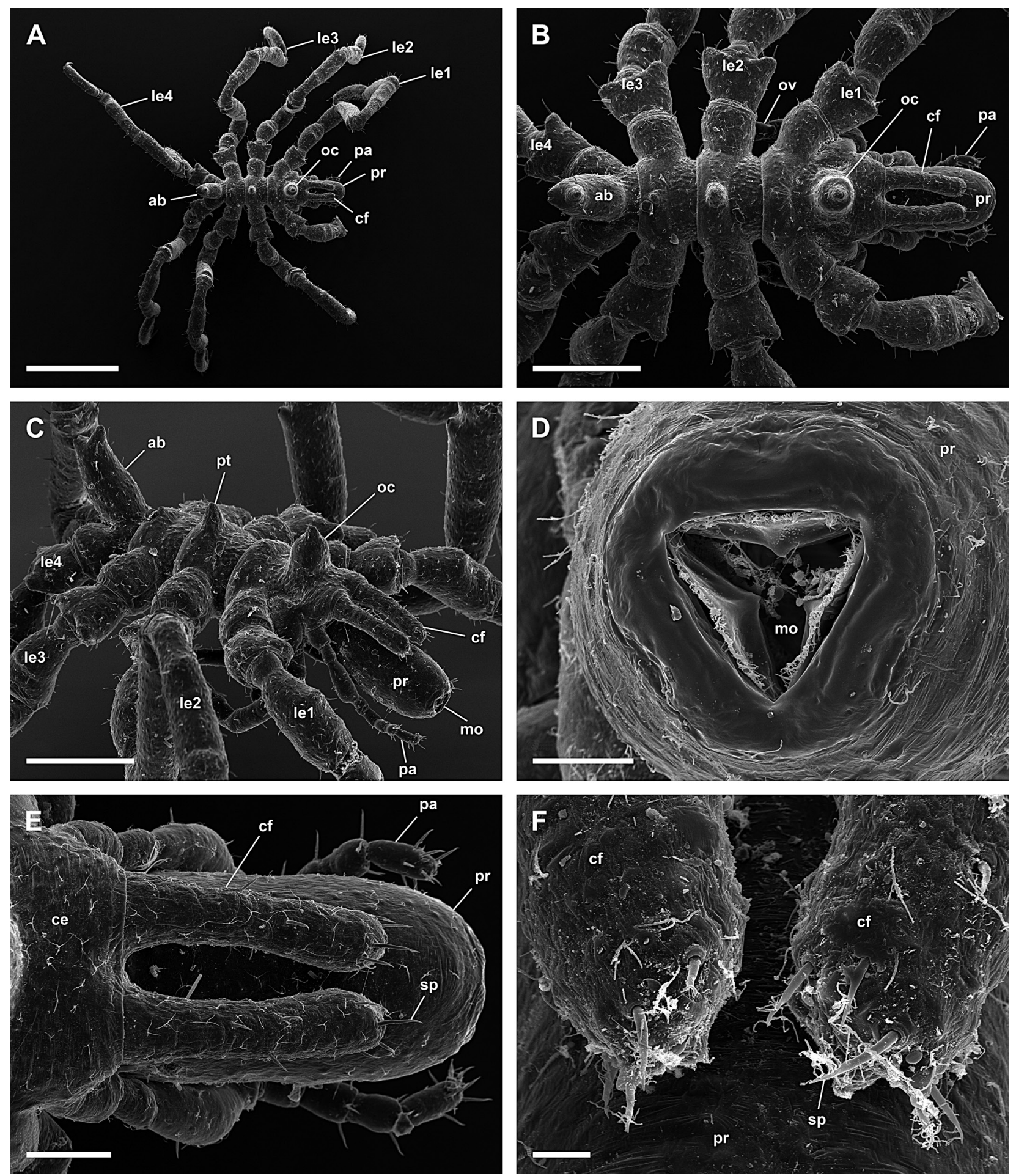

Figure 22. Trygaeus communis, male; A: Dorsal view; scale $1 \mathrm{~mm}$; B: Dorsal view of trunk; scale $400 \mu \mathrm{m}$; C: Lateral view of trunk with protuberances on midline; scale $400 \mu \mathrm{m}$; D: Mouth opening; scale $40 \mu \mathrm{m}$; E: Dorsal view of proboscis and chelifore without chela; scale $100 \mu \mathrm{m} ; \mathbf{F}$ : Tip of chelifore without chela; scale $20 \mu \mathrm{m}$. 

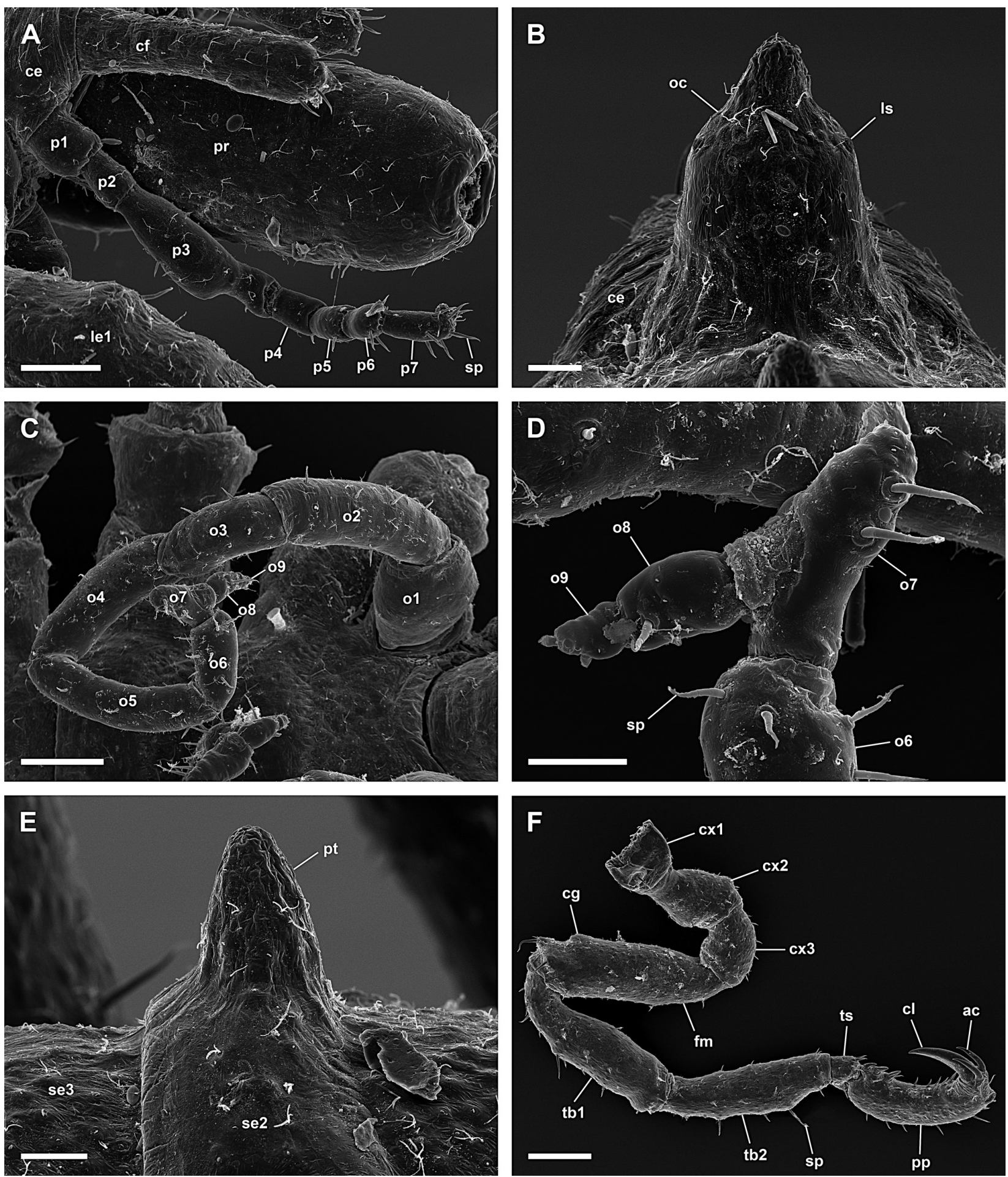

Figure 23. Trygaeus communis, male; A: Lateral view of proboscis and 7-articled right palp; scale $100 \mu \mathrm{m}$; B: Ocular tubercle with lateral sense organ; scale $40 \mu \mathrm{m} ; \mathbf{C}$ : Right 9-articled oviger; scale $100 \mu \mathrm{m}$; D: Distal articles of right oviger; scale $40 \mu \mathrm{m}$; E: Protuberance on midline of segment 2; scale $40 \mu \mathrm{m}$; F: Right 3rd leg; scale $200 \mu \mathrm{m}$. 

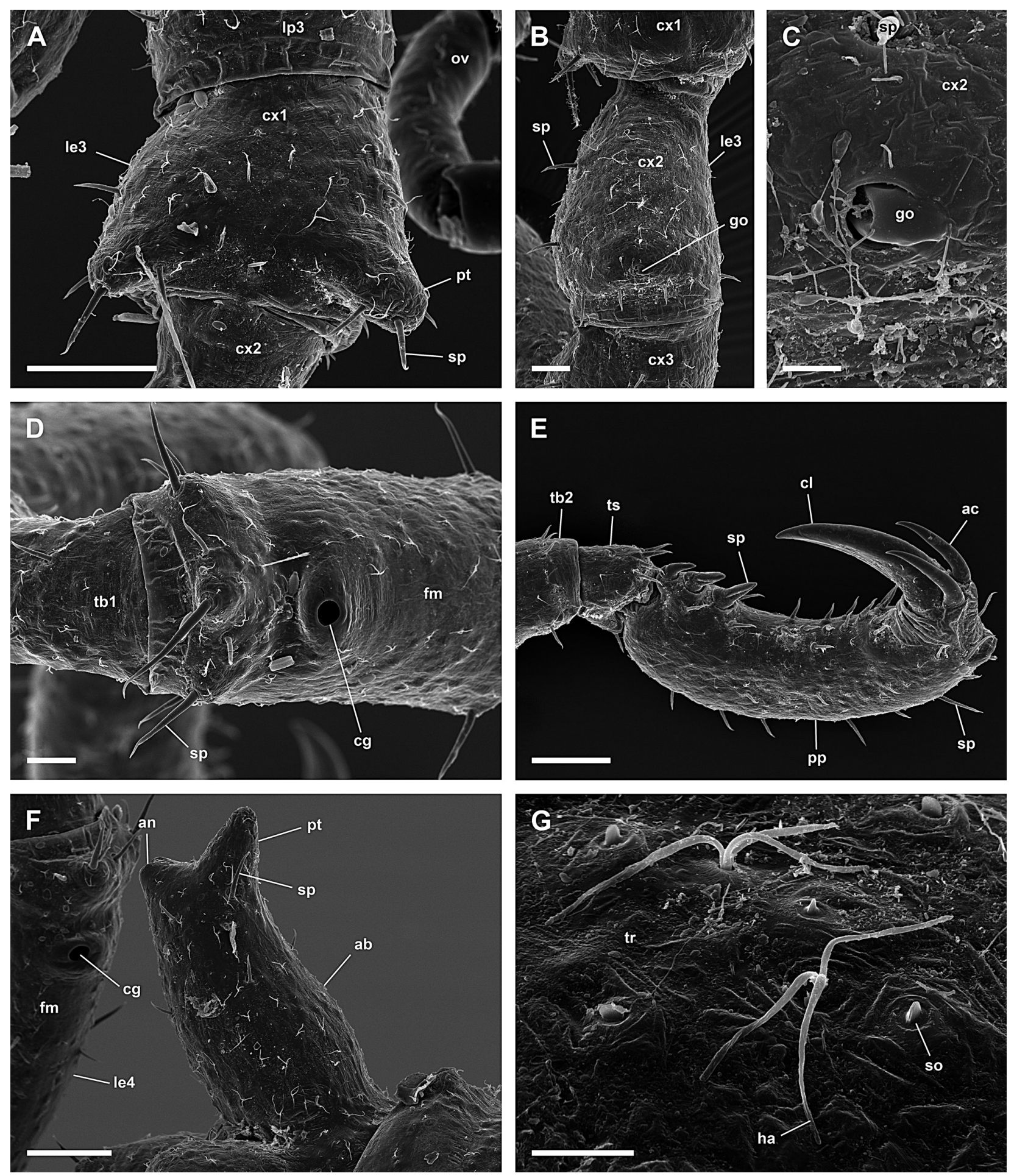

Figure 24. Trygaeus communis, male; A: Dorsal view of coxa 1 with protuberances on distal corners (right 3rd leg); scale $100 \mu \mathrm{m}$; B: Ventral view of coxa 2 with genital opening, distal is down (left 3rd leg); scale $40 \mu \mathrm{m}$; C: Genital opening; scale $10 \mu \mathrm{m}$; D: Cement gland on femur, distal is left (left 3rd leg); scale $40 \mu \mathrm{m}$; E: Tarsus, propodus, and claws (right 3rd leg); scale $100 \mu \mathrm{m}$; F: Abdomen with protuberance on tip; scale $100 \mu \mathrm{m} ; \mathbf{G}$ : Hairs and slit organs on dorsal side of trunk; scale $10 \mu \mathrm{m}$. 

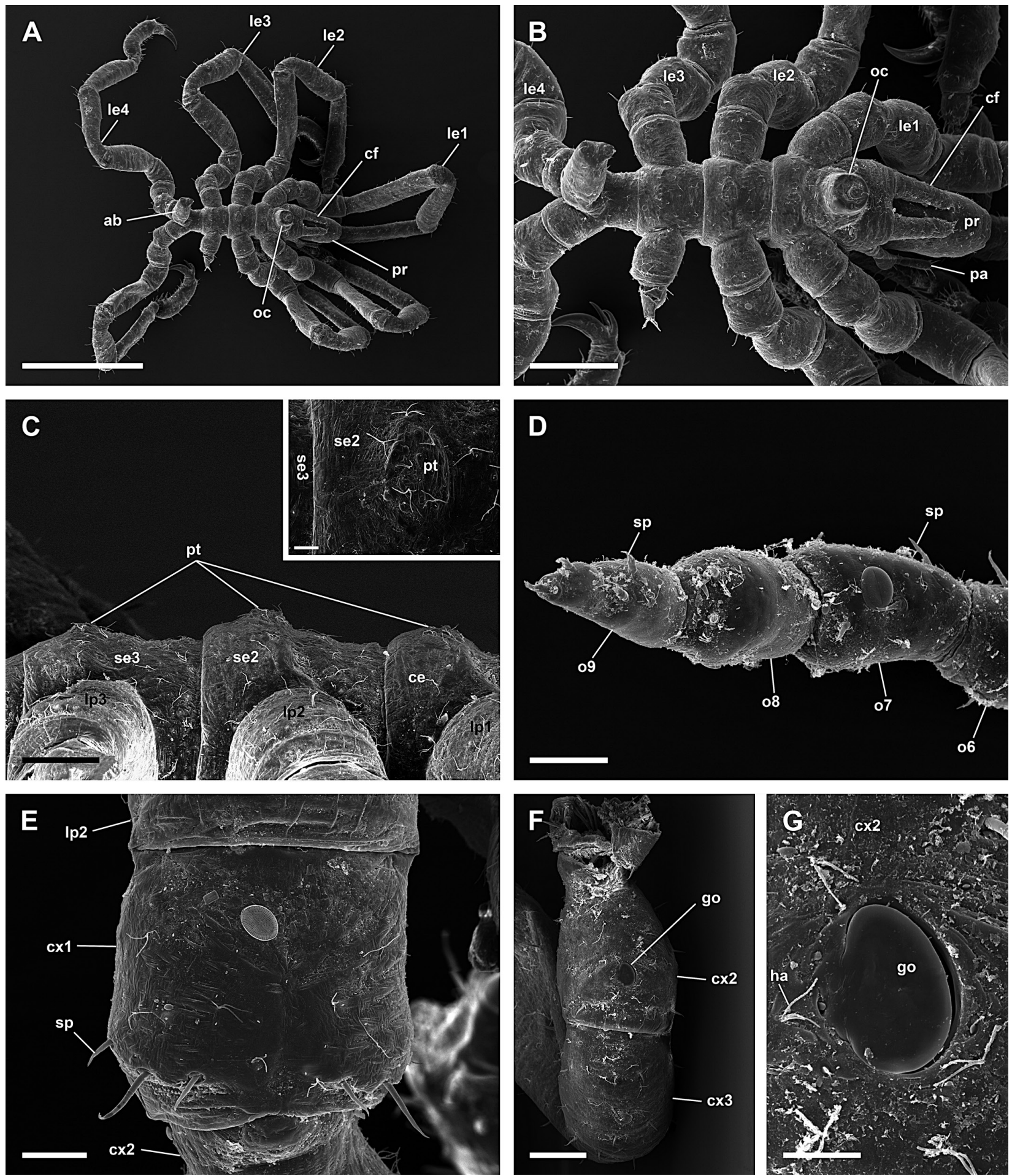

Figure 25. Trygaeus communis, female; A: Dorsal view; scale $1 \mathrm{~mm}$; B: Dorsal view of trunk; scale $300 \mu \mathrm{m}$; C: Lateral view of trunk with protuberances on midline, frontal is right; scale $100 \mu \mathrm{m}$; insert: Protuberance on segment 2; scale $20 \mu \mathrm{m}$; D: Distal article of oviger; scale $40 \mu \mathrm{m} ; \mathbf{E}$ : Dorsal view of coxa 1 without protuberances on distal corners (right $2 \mathrm{nd} \mathrm{leg}$ ); scale $40 \mu \mathrm{m} ; \mathbf{F}$ : Ventral view of coxa 2 with genital opening, distal is down (right 3rd leg); scale $100 \mu \mathrm{m}$; G: Genital opening; scale $20 \mu \mathrm{m}$. 

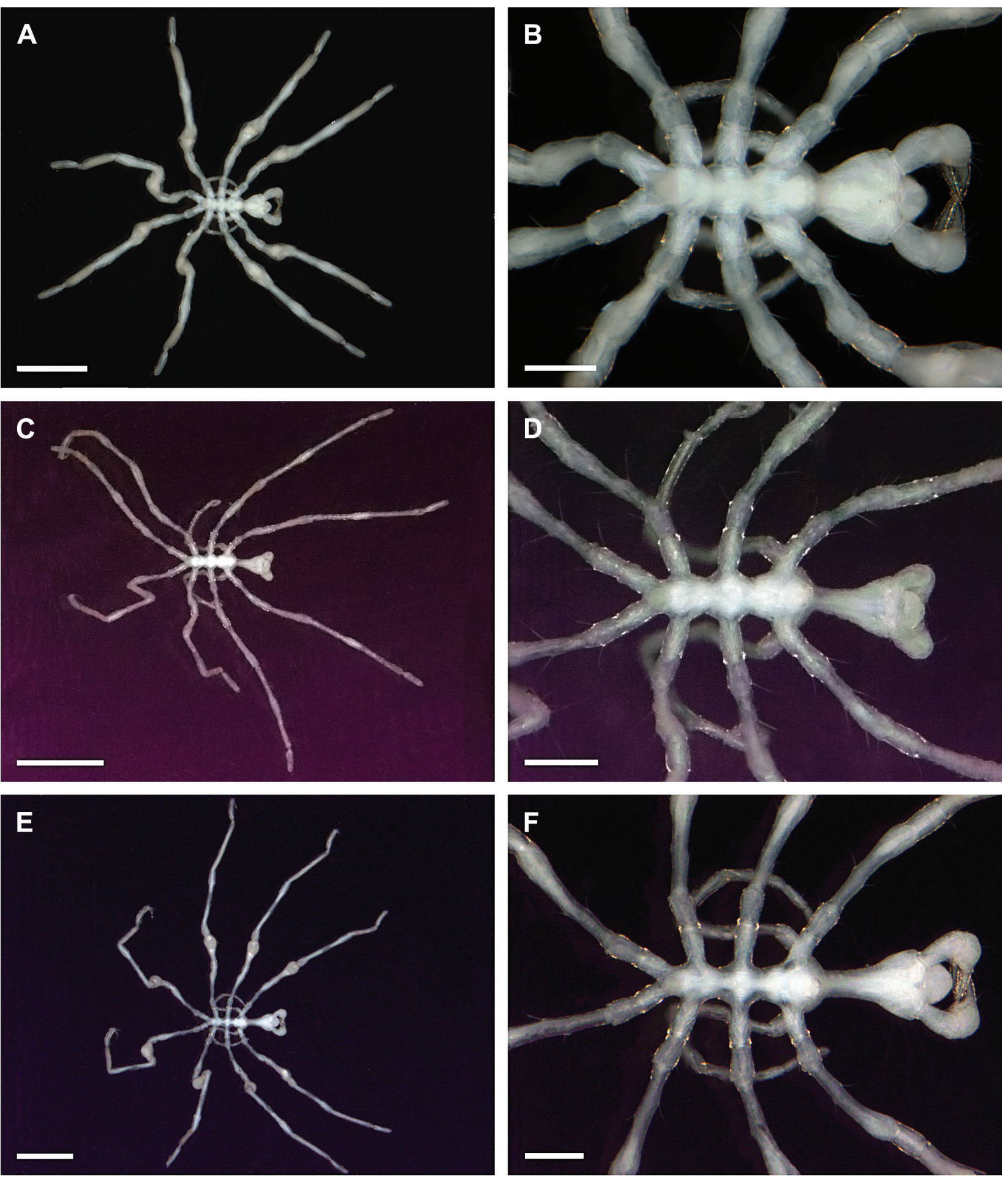

Figure 26. Callipallenidae 1; A, B: Callipallene emaciata, female, dorsal view; scales $1 \mathrm{~mm}$ and $250 \mu \mathrm{m}$, respectively; C, D: Callipallene phantoma, male, dorsal view; scales $1 \mathrm{~mm}$ and $250 \mu \mathrm{m}$, respectively; E, F: Callipallene producta, female, dorsal view; scales $1 \mathrm{~mm}$ and $250 \mu \mathrm{m}$, respectively. 

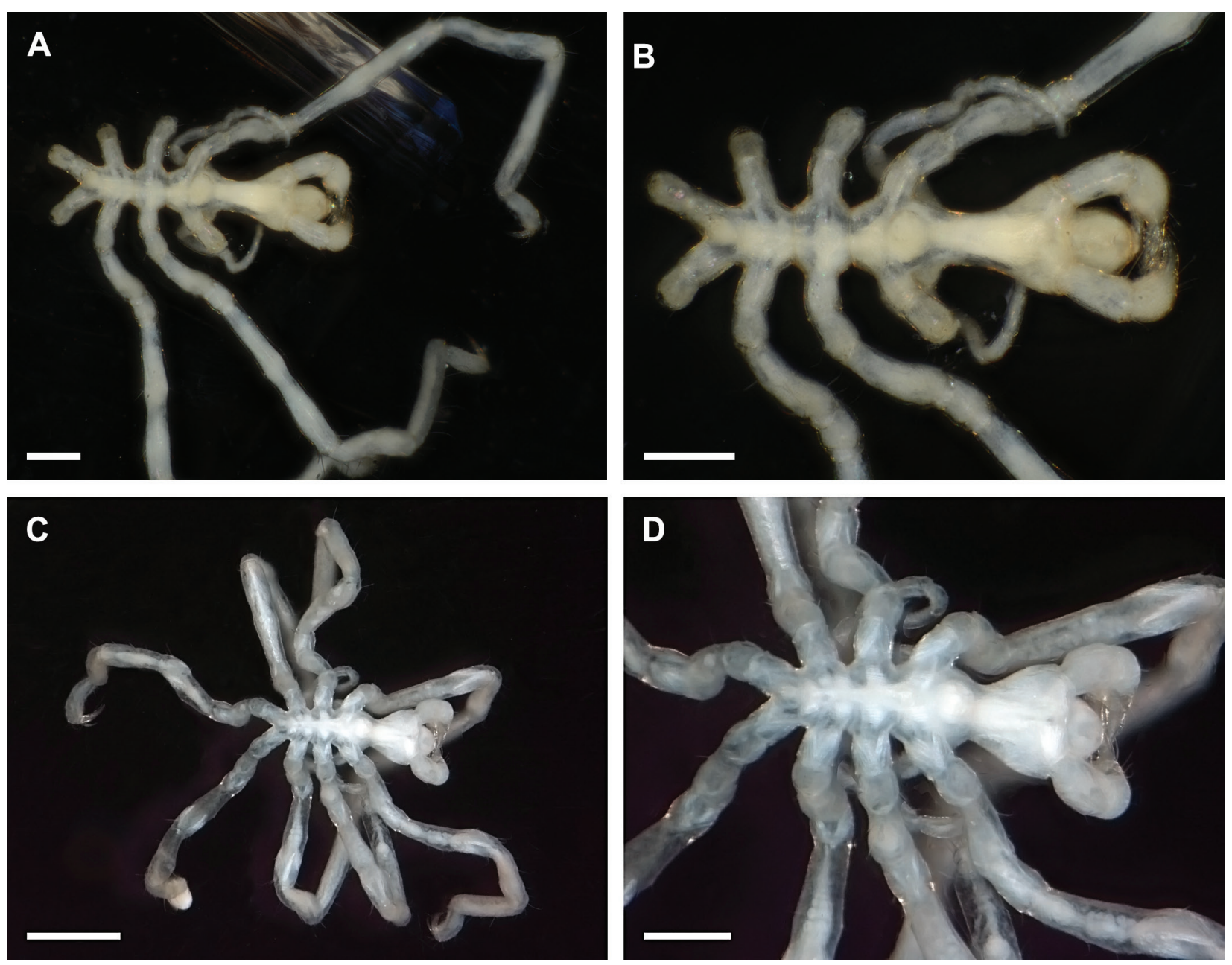

Figure 27. Callipallenidae 2; A, B: Callipallene spectrum, male, dorsal view; scales $200 \mu \mathrm{m}$; C, D: Callipallene tiberi, female, dorsal view; scales $500 \mu \mathrm{m}$ and $250 \mu \mathrm{m}$, respectively. 

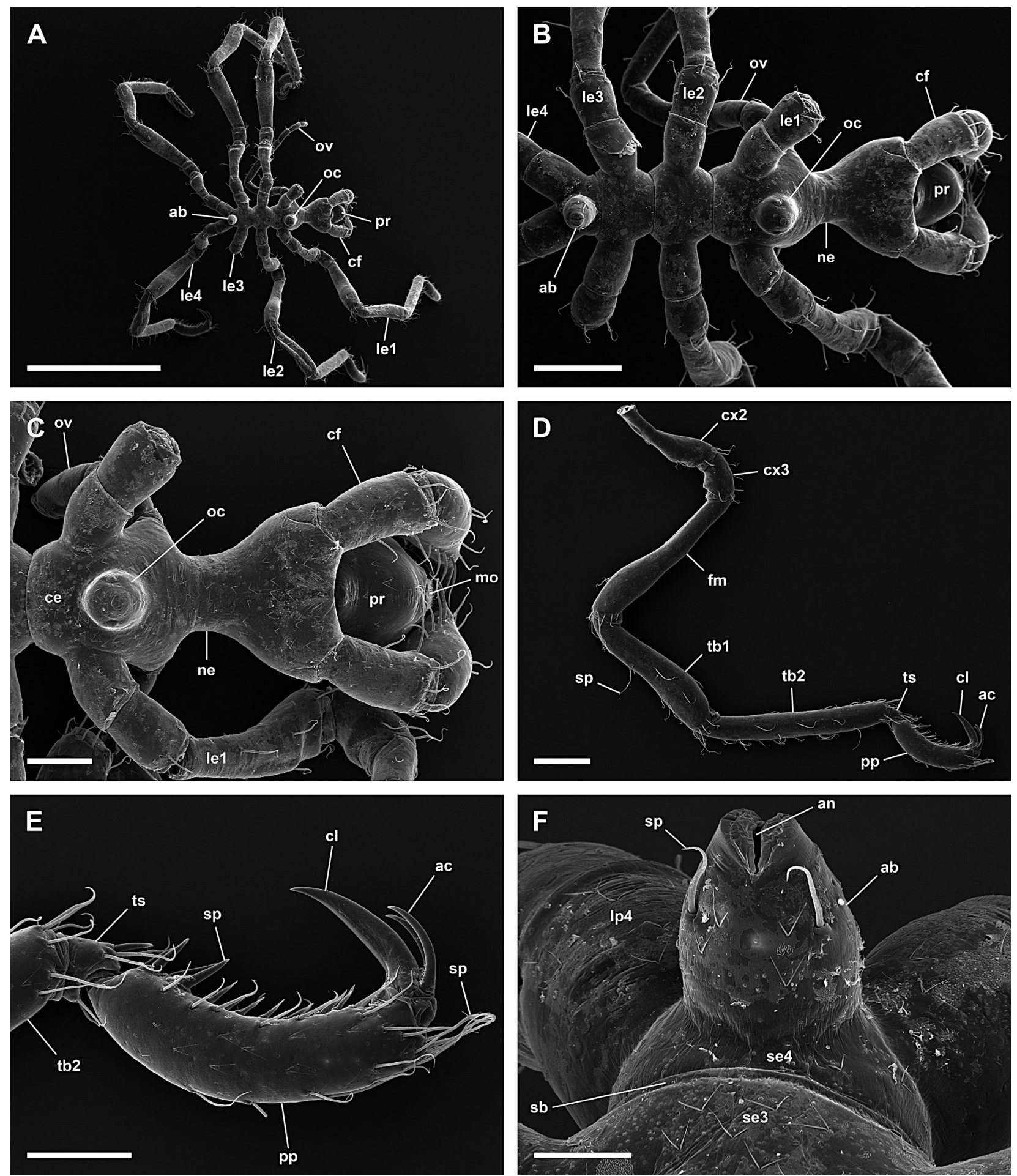

Figure 28. Callipallene emaciata, male; A: Dorsal view; scale $1 \mathrm{~mm}$; B: Dorsal view of trunk; scale $200 \mu \mathrm{m}$; C: Dorsal view of cephalon with rather slender neck; scale $100 \mu \mathrm{m}$; D: Right 3rd leg; scale $100 \mu \mathrm{m}$; E: Tarsus, strongly curved propodus, and claw, auxiliary claws about half as long as claw (right 3rd leg); scale $100 \mu \mathrm{m}$; F: Segment 3, 4 and abdomen; scale $40 \mu \mathrm{m}$. 

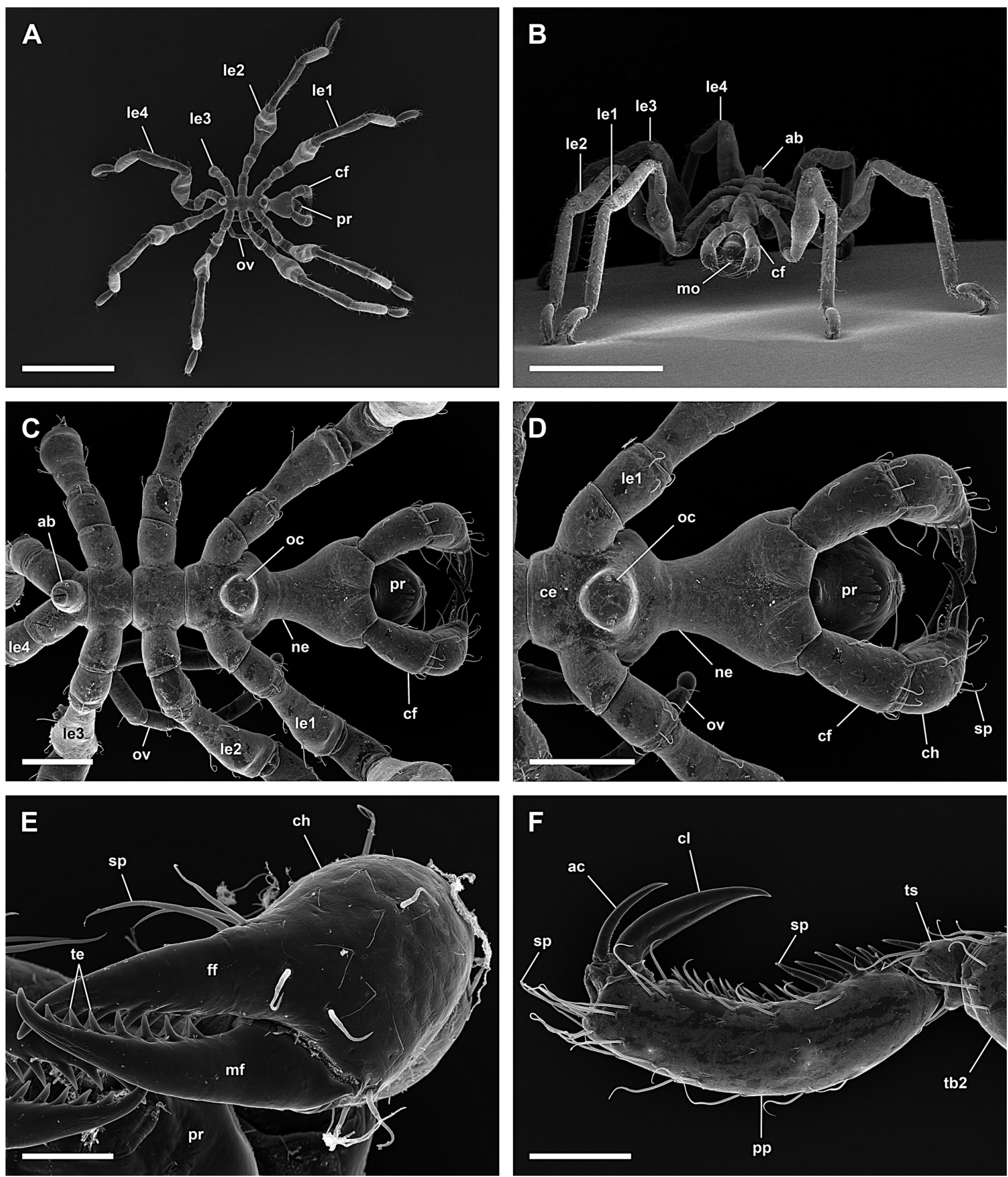

Figure 29. Callipallene emaciata, female; A: Dorsal view; scale $1 \mathrm{~mm}$; B: Frontal view; scale $1 \mathrm{~mm}$; C: Dorsal view of trunk; scale $200 \mu \mathrm{m}$; D: Dorsal view of cephalon with rather slender neck; scale $200 \mu \mathrm{m}$; E: Left chela, dorsal is up; scale $40 \mu \mathrm{m} ; \mathbf{F}$ : Tarsus, strongly curved propodus, and claw, auxiliary claws about half as long as claw (left $3 \mathrm{rd} \mathrm{leg}$ ); scale $100 \mu \mathrm{m}$. 

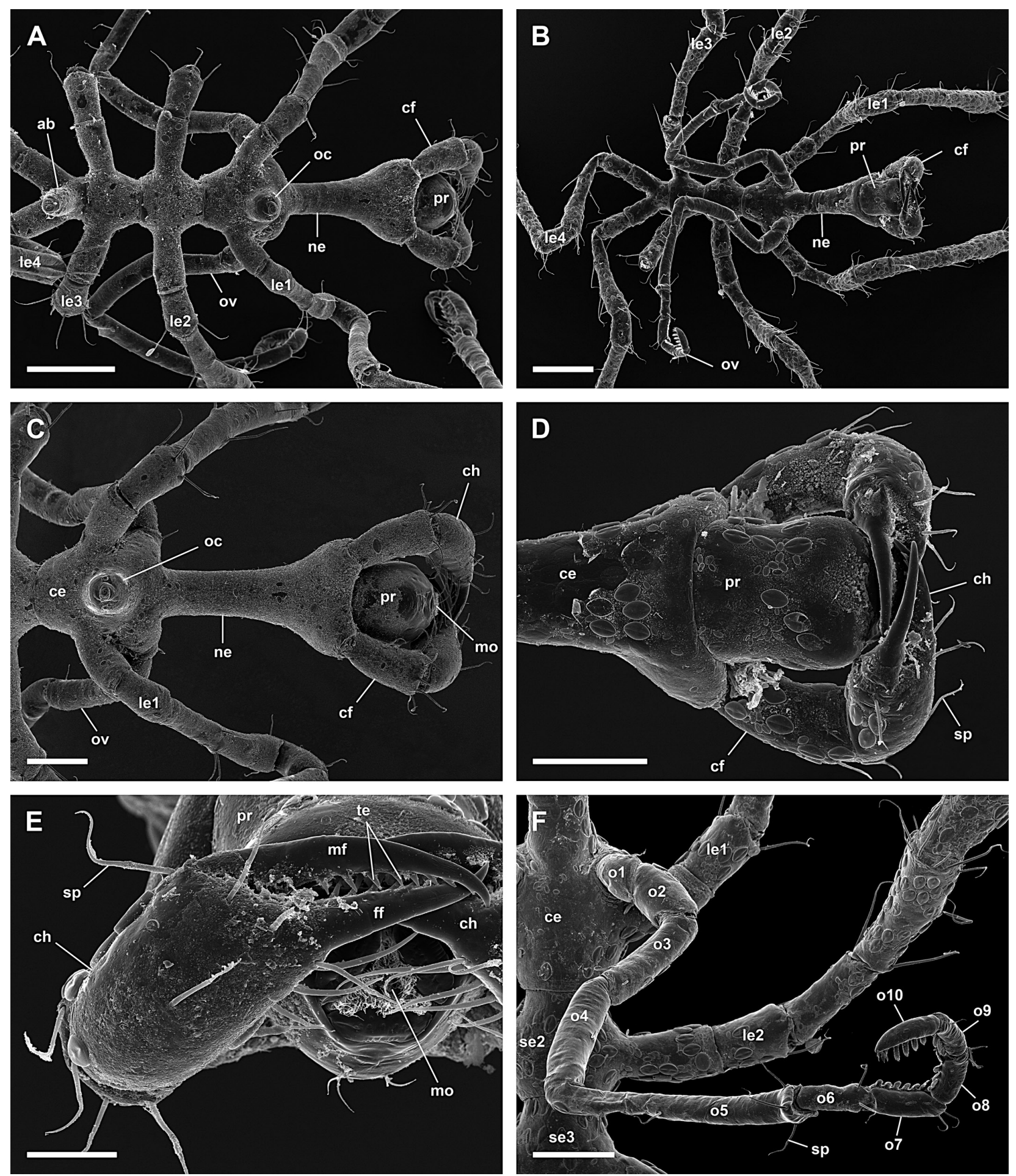

Figure 30. Callipallene phantoma, male; A: Dorsal view of trunk; scale $200 \mu \mathrm{m}$; B: Ventral view of trunk; scale $200 \mu \mathrm{m}$; C: Dorsal view of cephalon with very long and slender neck; scale $100 \mu \mathrm{m} ; \mathbf{D}$ : Ventral view of proboscis; scale $100 \mu \mathrm{m}$; E: Left chela, dorsal is down; scale $40 \mu \mathrm{m}$; F: Left 10-articled oviger; scale $100 \mu \mathrm{m}$. 

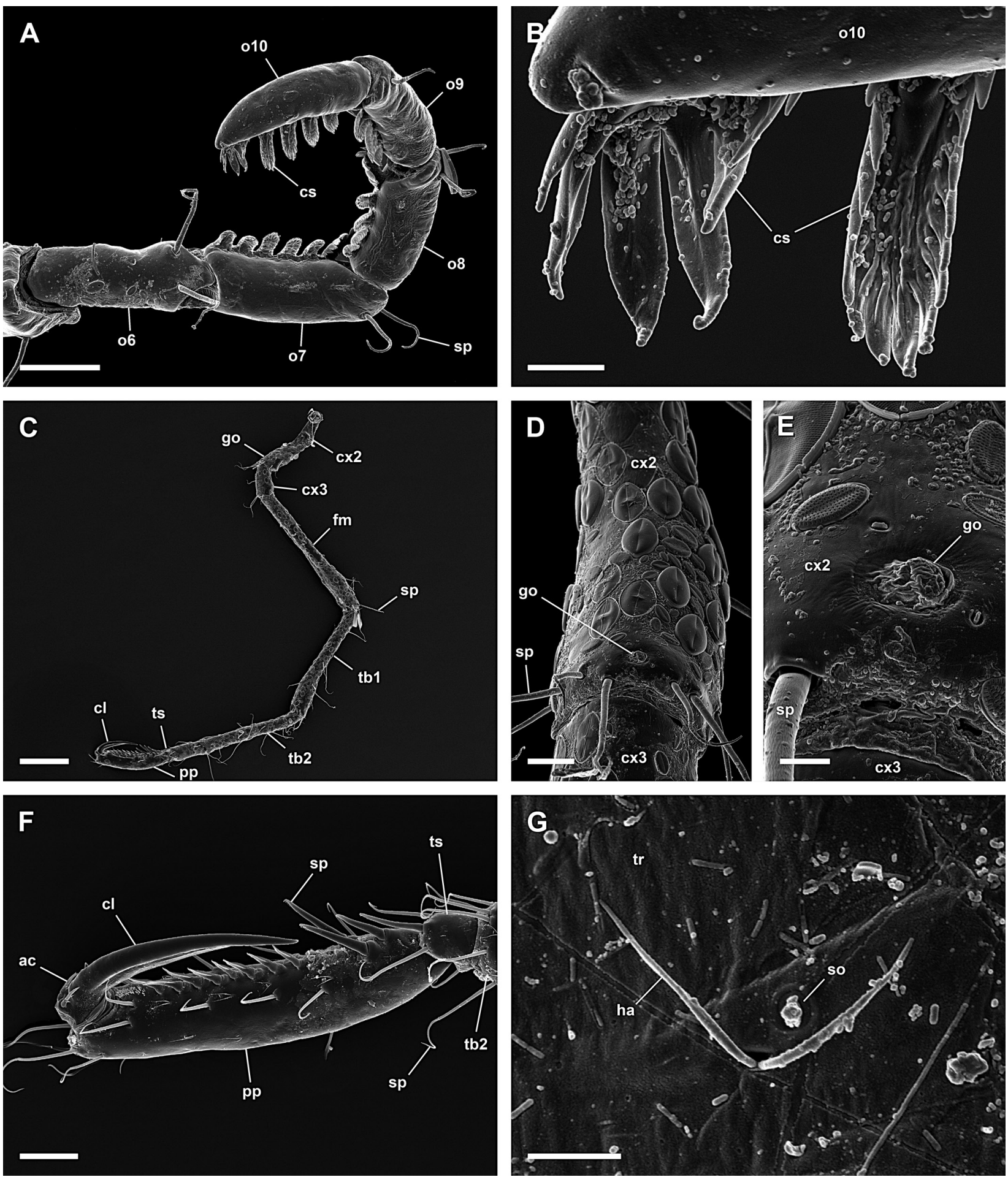

Figure 31. Callipallene phantoma, male; A: Distal articles of left oviger with compound spines; scale $40 \mu \mathrm{m}$; B: Compound spines on last oviger-article; scale $5 \mu \mathrm{m}$; C: Left 3rd leg; scale $200 \mu \mathrm{m}$; D: Ventral view of coxa 2 with genital opening, distal is down (right 2nd leg); scale $20 \mu \mathrm{m}$; E: Genital opening; scale $5 \mu \mathrm{m}$; F: Tarsus, straight propodus, and claw with very short auxiliary claws (right 3rd leg); scale $40 \mu \mathrm{m}$; G: Hair and slit organ on dorsal side of trunk; scale $5 \mu \mathrm{m}$. 

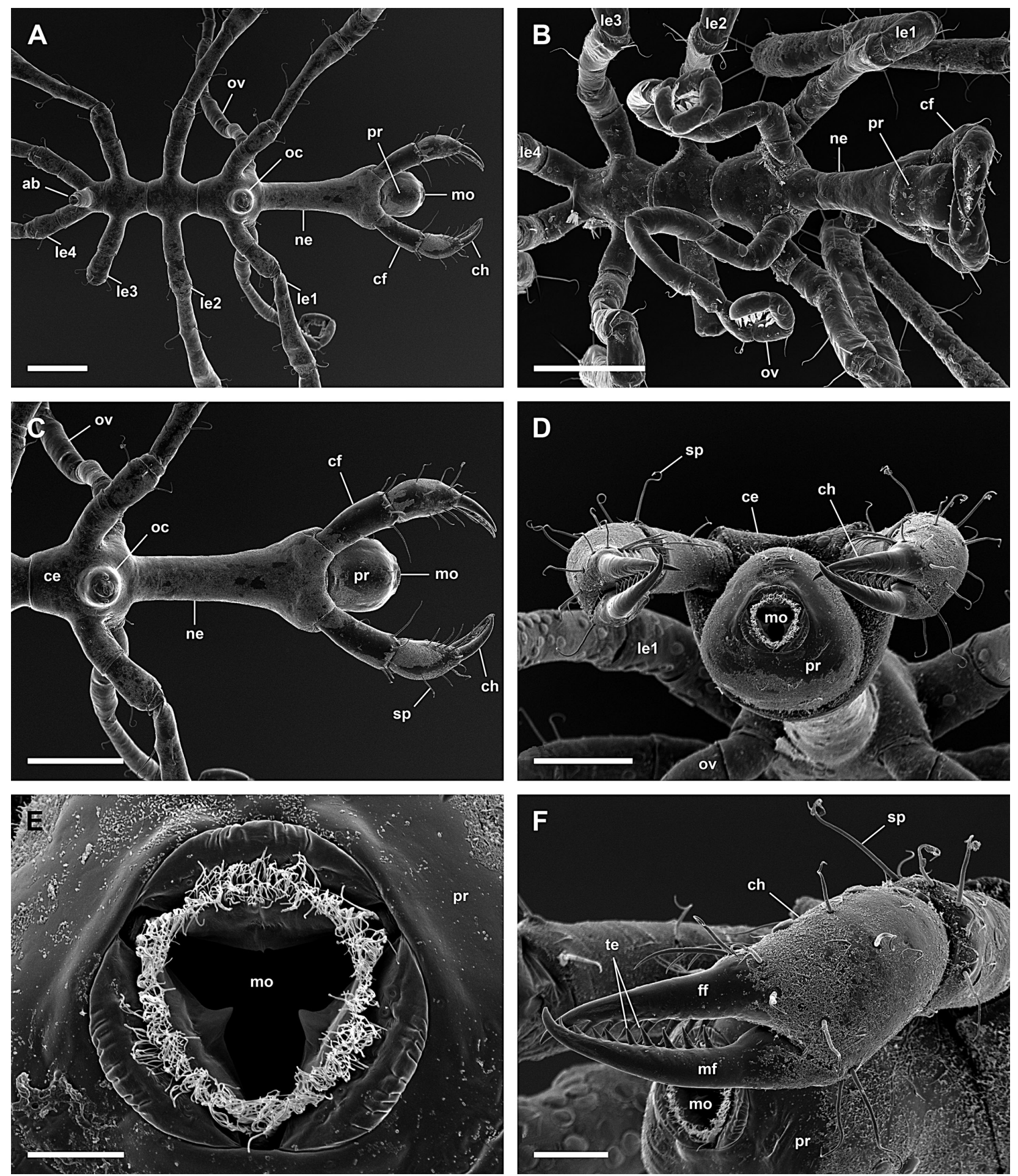

Figure 32. Callipallene phantoma, female; A: Dorsal view of trunk; scale $200 \mu \mathrm{m}$; B: Ventral view of trunk; scale $200 \mu \mathrm{m}$; C: Dorsal view of cephalon with very long and slender neck; scale $200 \mu \mathrm{m}$; D: Frontal view of cephalon; scale $100 \mu \mathrm{m}$; E: Mouth opening; scale $20 \mu \mathrm{m} ; \mathbf{F}$ : Left chela, dorsal is up; scale $40 \mu \mathrm{m}$. 

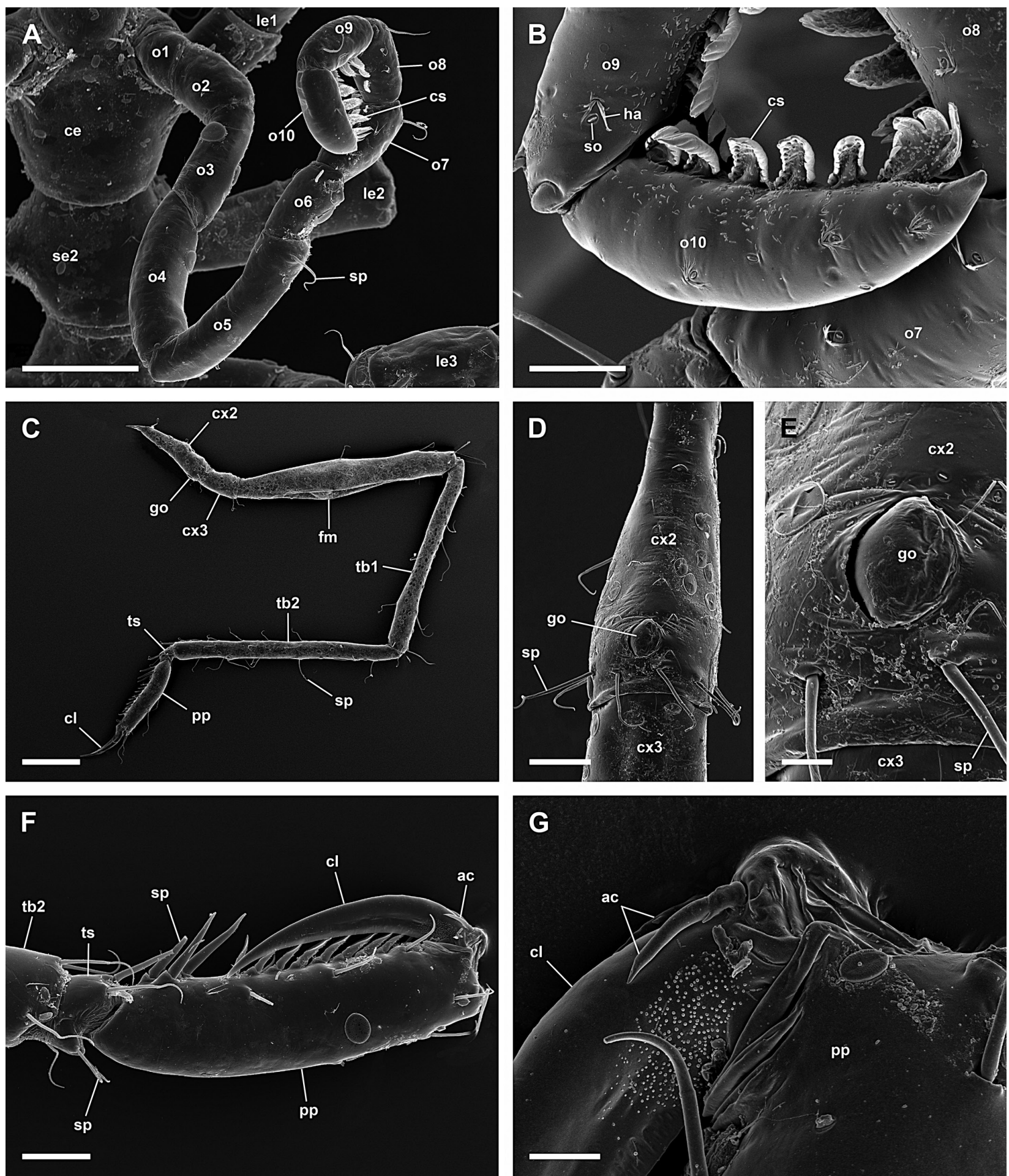

Figure 33. Callipallene phantoma, female; A: Right 10-articled oviger; scale $100 \mu \mathrm{m} ; \mathbf{B}$ : Distalmost oviger-articles with compound spines; scale $20 \mu \mathrm{m}$; C: Right 3rd leg; scale $200 \mu \mathrm{m}$; D: Ventral view of coxa 2 with genital opening, distal is down (left 4th leg); scale $40 \mu \mathrm{m}$; E: Genital opening; scale $10 \mu \mathrm{m}$; F: Tarsus, straight propodus, and claw with very short auxiliary claws (left 3rd leg); scale $40 \mu \mathrm{m}$; G: Small auxiliary claws; scale $10 \mu \mathrm{m}$. 

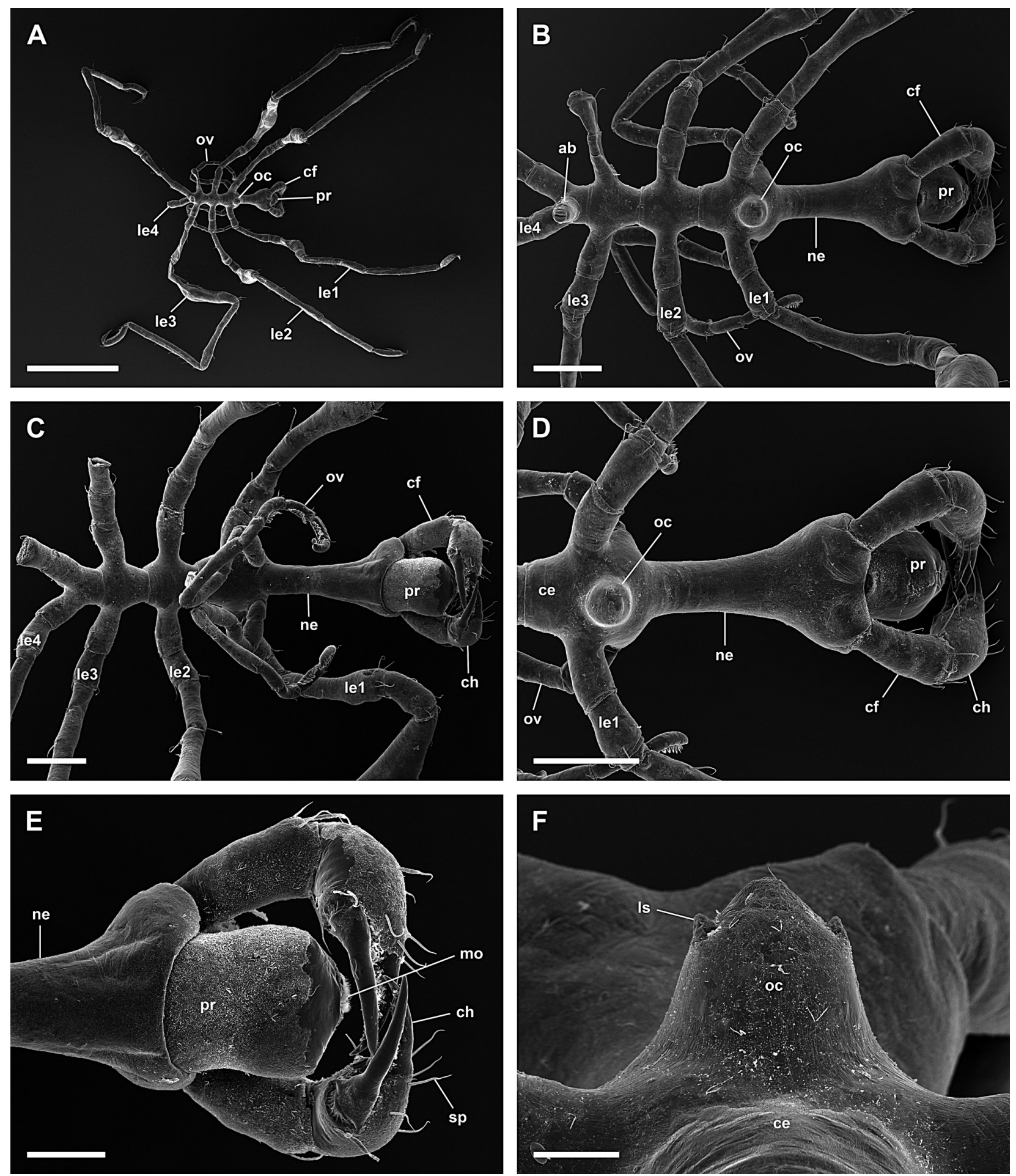

Figure 34. Callipallene producta, female; A: Dorsal view; scale $1 \mathrm{~mm}$; B: Dorsal view of trunk; scale $200 \mu \mathrm{m}$; C: Ventral view of trunk; scale $200 \mu \mathrm{m}$; D: Dorsal view of cephalon with very long and slender neck; scale $200 \mu \mathrm{m}$; $\mathbf{E}$ : Ventral view of proboscis; scale $100 \mu \mathrm{m} ; \mathbf{F}$ : Ocular tubercle with lateral sense organ; scale $40 \mu \mathrm{m}$. 

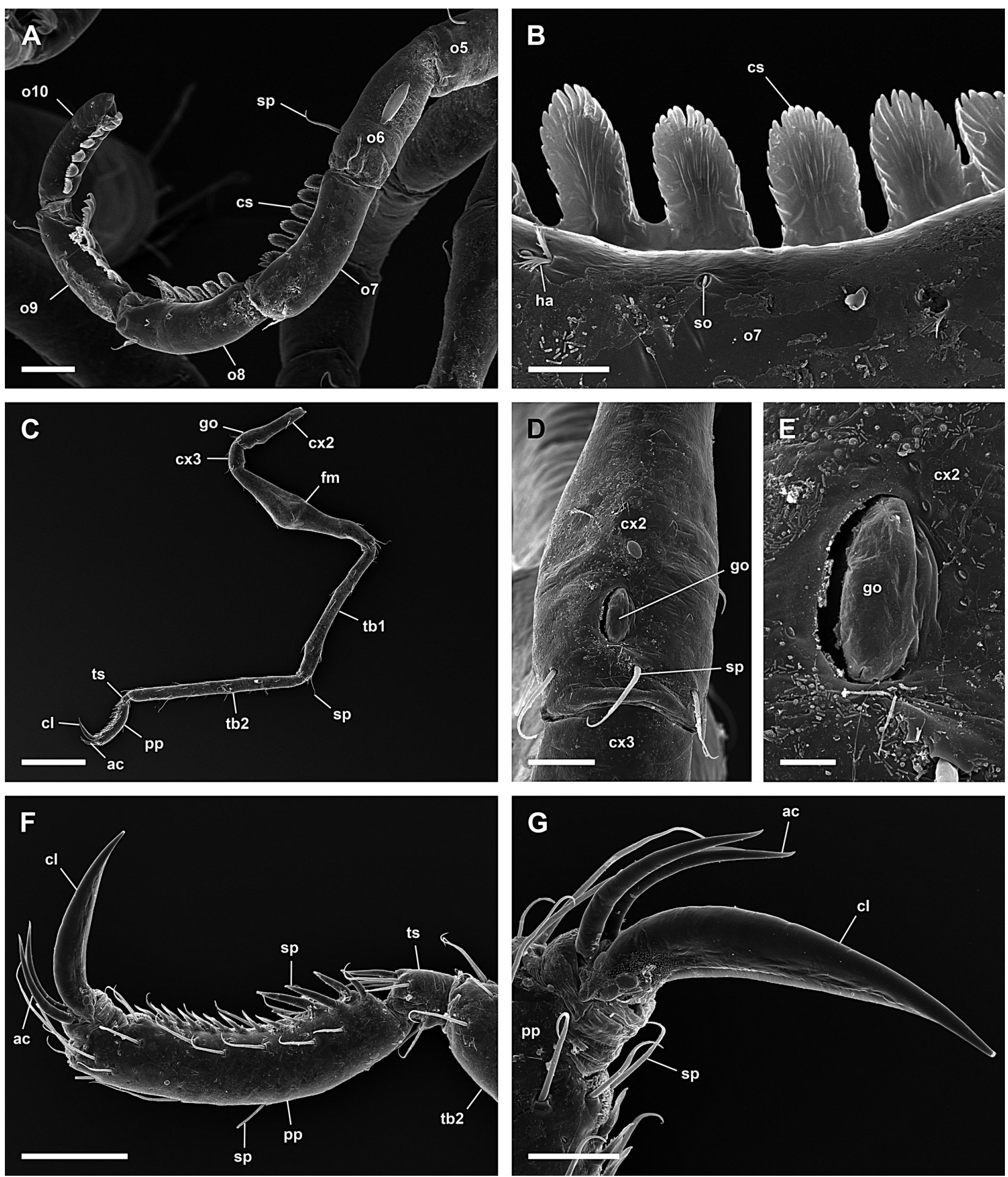

Figure 35. Callipallene producta, female; A: Distal article of right 10-articled oviger; scale $40 \mu \mathrm{m}$; B: Compound spines on oviger-article 7; scale $10 \mu \mathrm{m}$; C: Right 3rd leg; scale $400 \mu \mathrm{m}$; D: Ventral view of coxa 2 with genital opening, distal is down (left 3rd leg); scale $40 \mu \mathrm{m}$; E: Genital opening; scale $10 \mu \mathrm{m}$; F: Tarsus, slightly curved propodus, and claw with very long auxiliary claws (right 3rd leg); scale $100 \mu \mathrm{m}$; G: Claw and auxiliary claws; scale $40 \mu \mathrm{m}$. 

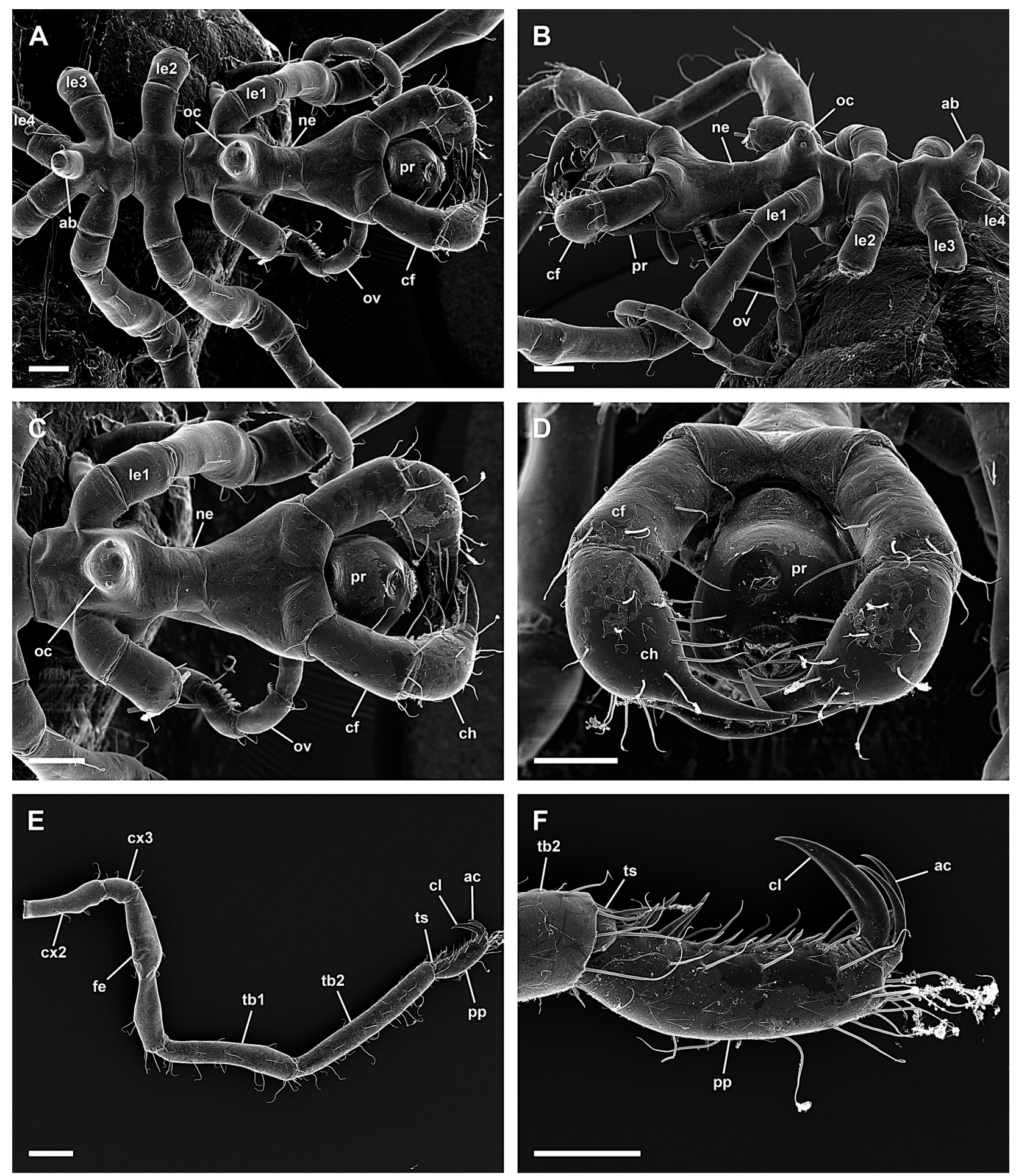

Figure 36. Callipallene spectrum, male; A: Dorsal view of trunk; scale $100 \mu \mathrm{m}$; B: Lateral view of trunk; scale $100 \mu \mathrm{m}$; C: Dorsal view of cephalon; scale $100 \mu \mathrm{m}$; D: Cephalon with proboscis and chelifore; scale $100 \mu \mathrm{m}$; E: Left 3rd leg; scale $200 \mu \mathrm{m}$; F: Tibia2, tarsus, propodus, and claws (left 3rd leg); scale $100 \mu \mathrm{m}$. 

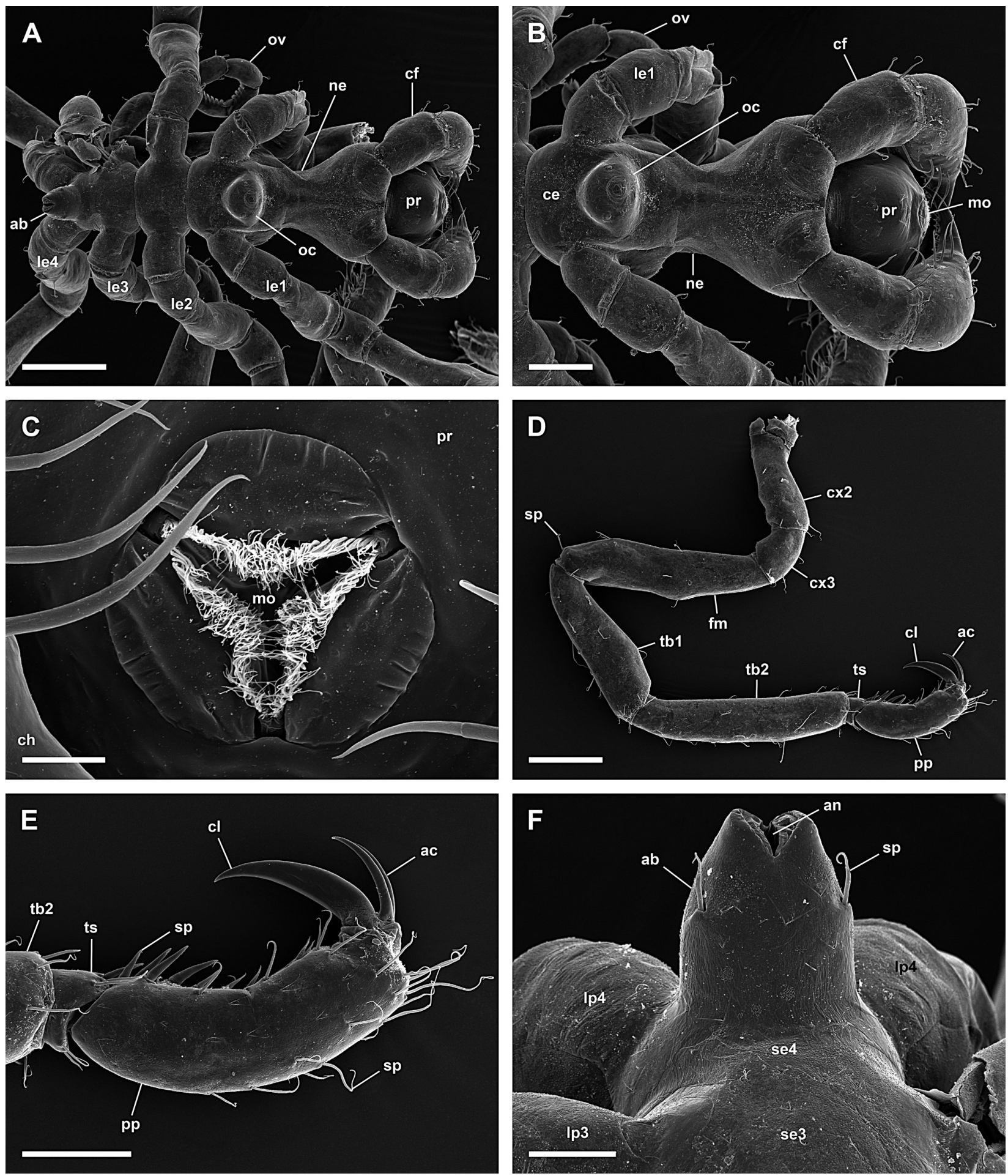

Figure 37. Callipallene tiberi, female; A: Dorsal view of trunk; scale $200 \mu \mathrm{m}$; B: Dorsal view of cephalon with very short neck; scale $100 \mu \mathrm{m}$; C: Mouth opening; scale $20 \mu \mathrm{m}$; D: Left 3rd leg; scale $200 \mu \mathrm{m}$; E: Tarsus, strongly curved propodus, and claw, auxiliary claws about half as long as claw (left 3rd leg); scale $100 \mu \mathrm{m}$; F: Segment 3, 4 and abdomen; scale $40 \mu \mathrm{m}$. 

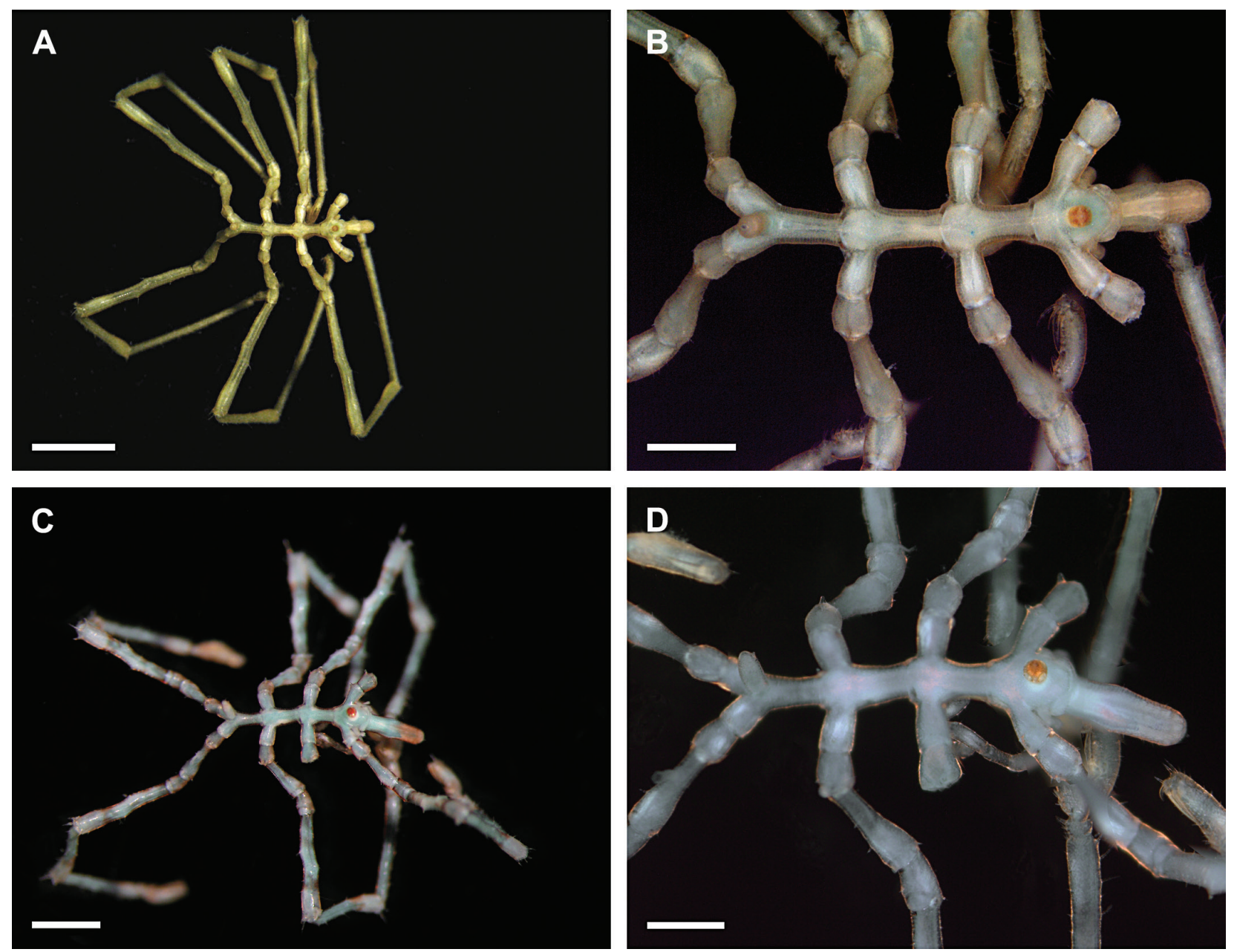

Figure 38. Endeidae; A, B: Endeis charybdaea, male, dorsal view; scales $3 \mathrm{~mm}$ and $1 \mathrm{~mm}$, respectively; C, D: Endeis spinosa, male, dorsal view; scales $1 \mathrm{~mm}$ and $500 \mu \mathrm{m}$, respectively. 

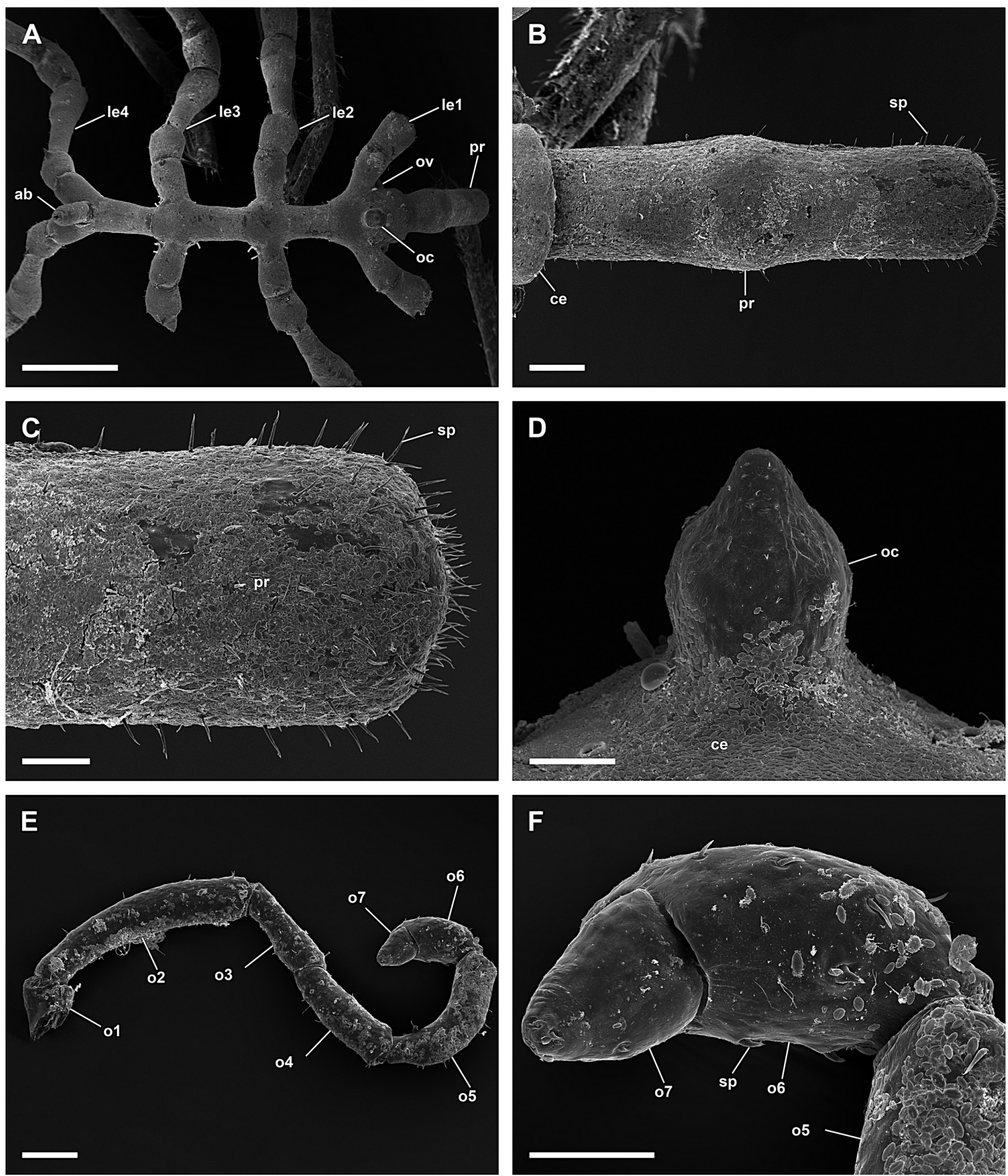

Figure 39. Endeis charybdaea, male; A: Dorsal view of trunk; scale $1 \mathrm{~mm}$; B: Dorsal view of proboscis; scale $200 \mu \mathrm{m}$; C: Tip of proboscis with numerous spines around mouth; scale $100 \mu \mathrm{m}$; D: Ocular tubercle; scale $100 \mu \mathrm{m}$; E: 7-articled oviger; scale $200 \mu \mathrm{m}$; F: Distal articles of oviger; scale $100 \mu \mathrm{m}$. 

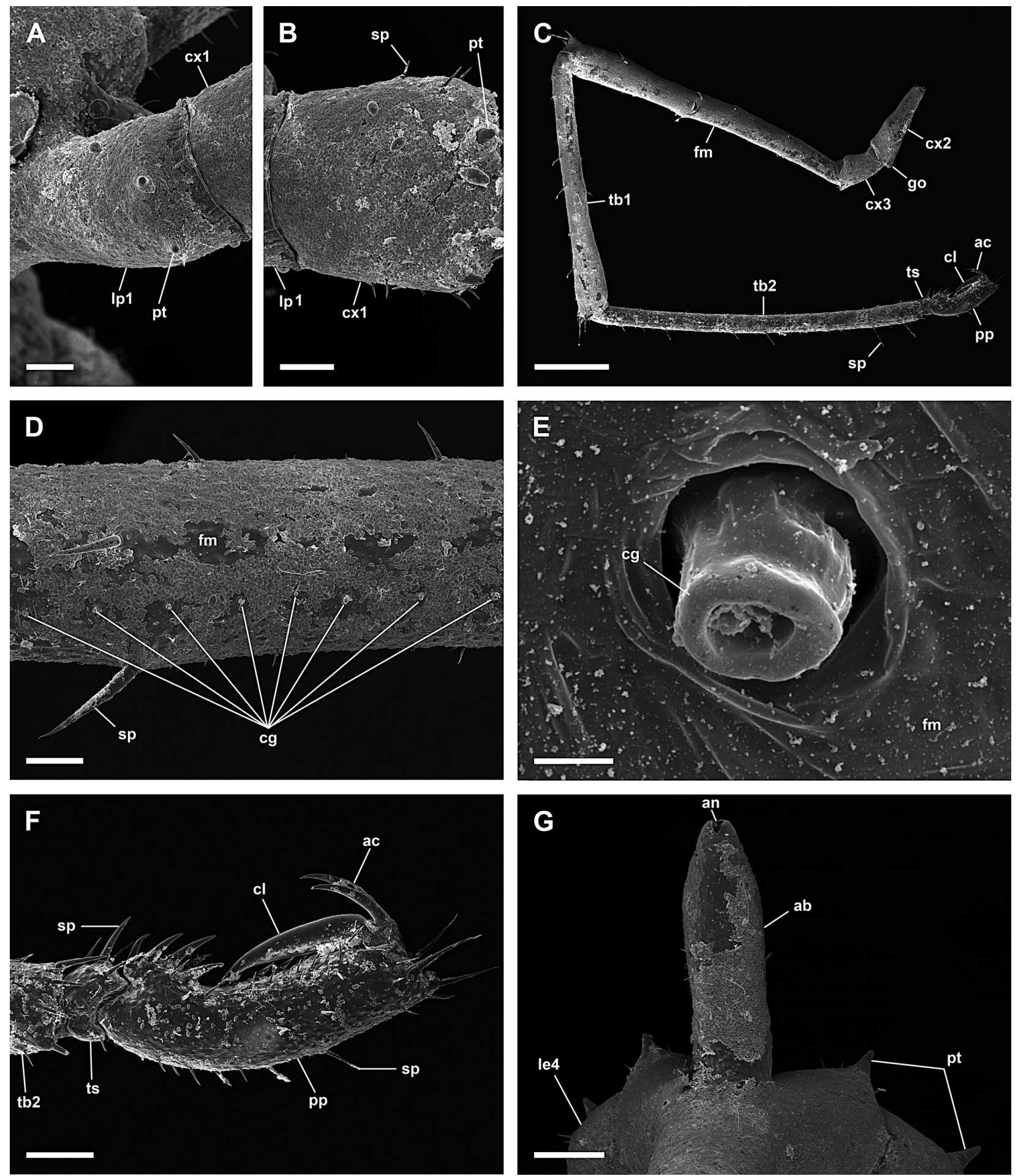

Figure 40. Endeis charybdaea, male; A: Right lateral process 1 with 2 protuberances; scale $100 \mu \mathrm{m}$; B: Coxa 1 of right 1 st leg with 2 protuberances; scale $100 \mu \mathrm{m}$; C: Right 3rd leg; scale $1 \mathrm{~mm}$; D: Femur with 8 of overall 24 cement glands (left 3rd leg); scale $100 \mu \mathrm{m}$; E: Single cement gland; scale $5 \mu \mathrm{m}$; F: Tarsus, propodus, and claws (right 3rd leg); scale $200 \mu \mathrm{m}$; G: Abdomen; scale $200 \mu \mathrm{m}$. 

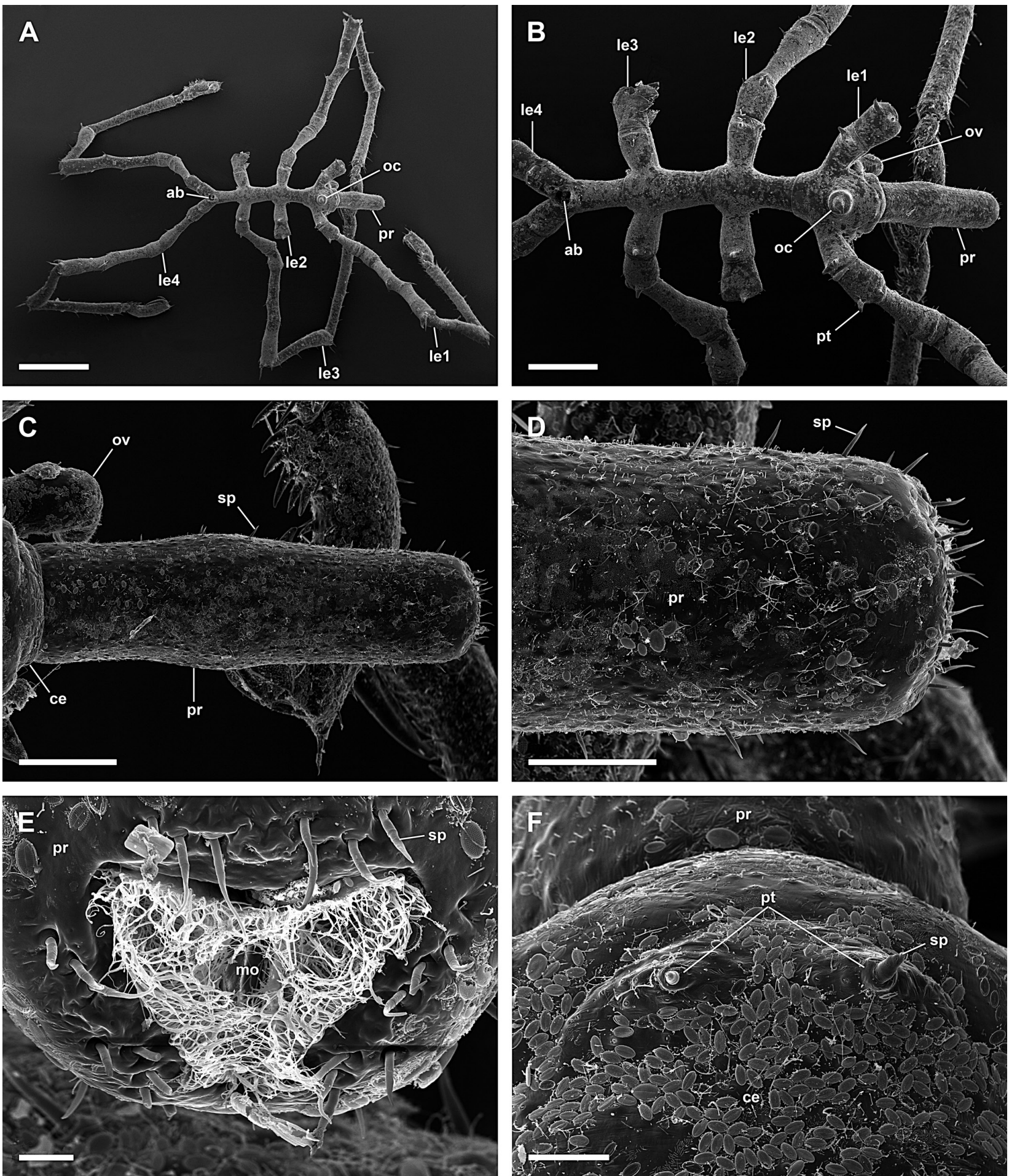

Figure 41. Endeis spinosa, male; A: Dorsal view, abdomen lost due to preparation; scale $1 \mathrm{~mm}$; B: Dorsal view of trunk; scale $400 \mu \mathrm{m} ; \mathbf{C}$ : Dorsal view of proboscis; scale $200 \mu \mathrm{m}$; D: Tip of proboscis with few spines around mouth; scale $100 \mu \mathrm{m}$; E: Mouth opening; scale $20 \mu \mathrm{m} ; \mathbf{F}$ : Cephalon with small protuberances with spine and without chelifores; scale $40 \mu \mathrm{m}$. 

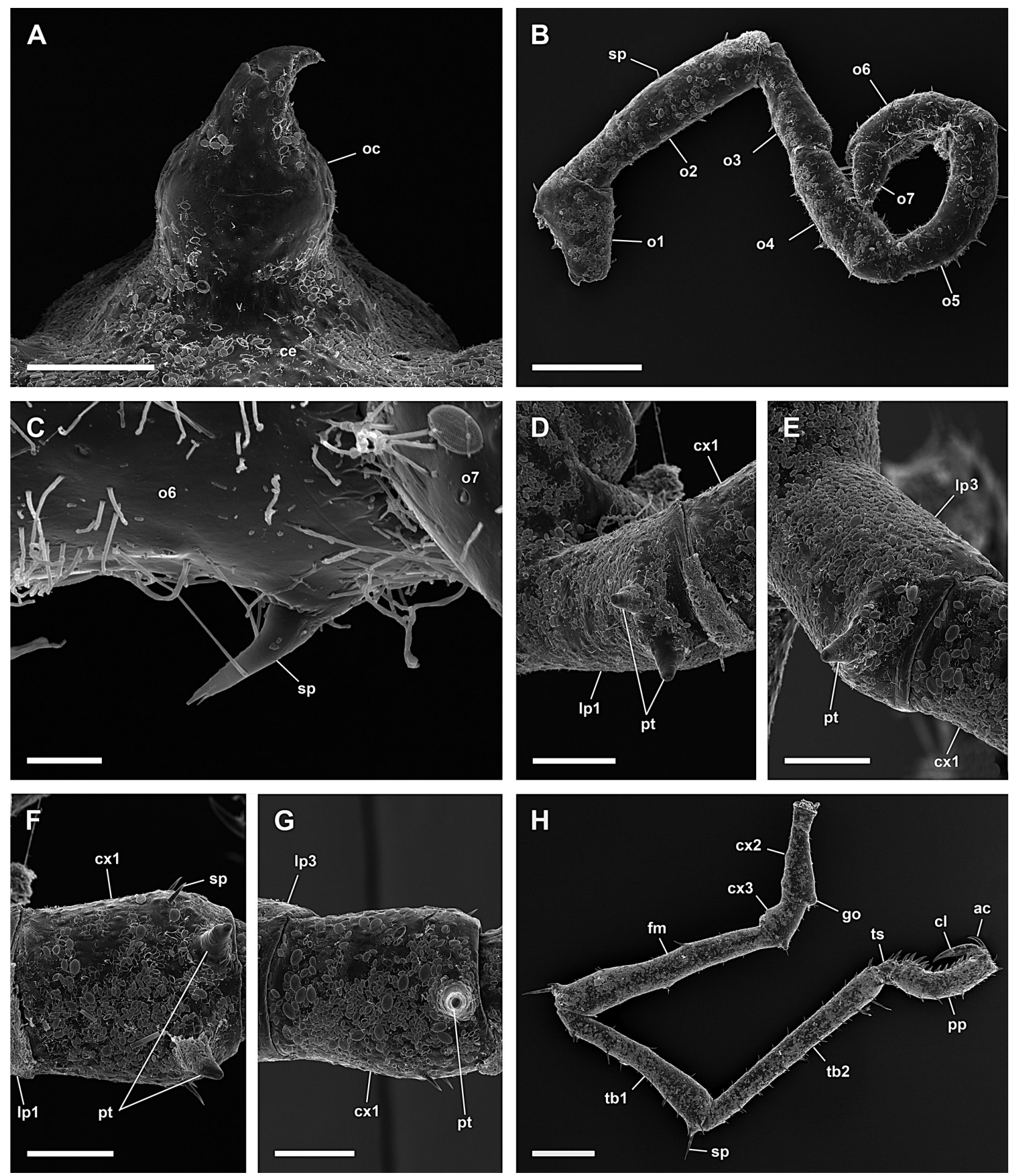

Figure 42. Endeis spinosa, male; A: Ocular tubercle; scale $100 \mu \mathrm{m}$; B: 7-articled oviger; scale $200 \mu \mathrm{m}$; C: Small spine on oviger-article 6; scale $10 \mu \mathrm{m}$; D: Right lateral process 1 with 2 protuberances; scale $100 \mu \mathrm{m}$; E: Right lateral process 3 with 1 protuberance; scale $100 \mu \mathrm{m}$; F: Coxa 1 of right 1 st leg with 2 protuberances; scale $100 \mu \mathrm{m}$; G: Coxa 1 of right $3 \mathrm{rd}$ leg with 1 protuberance; scale $100 \mu \mathrm{m} ;$ H: Left 3rd leg; scale $400 \mu \mathrm{m}$. 

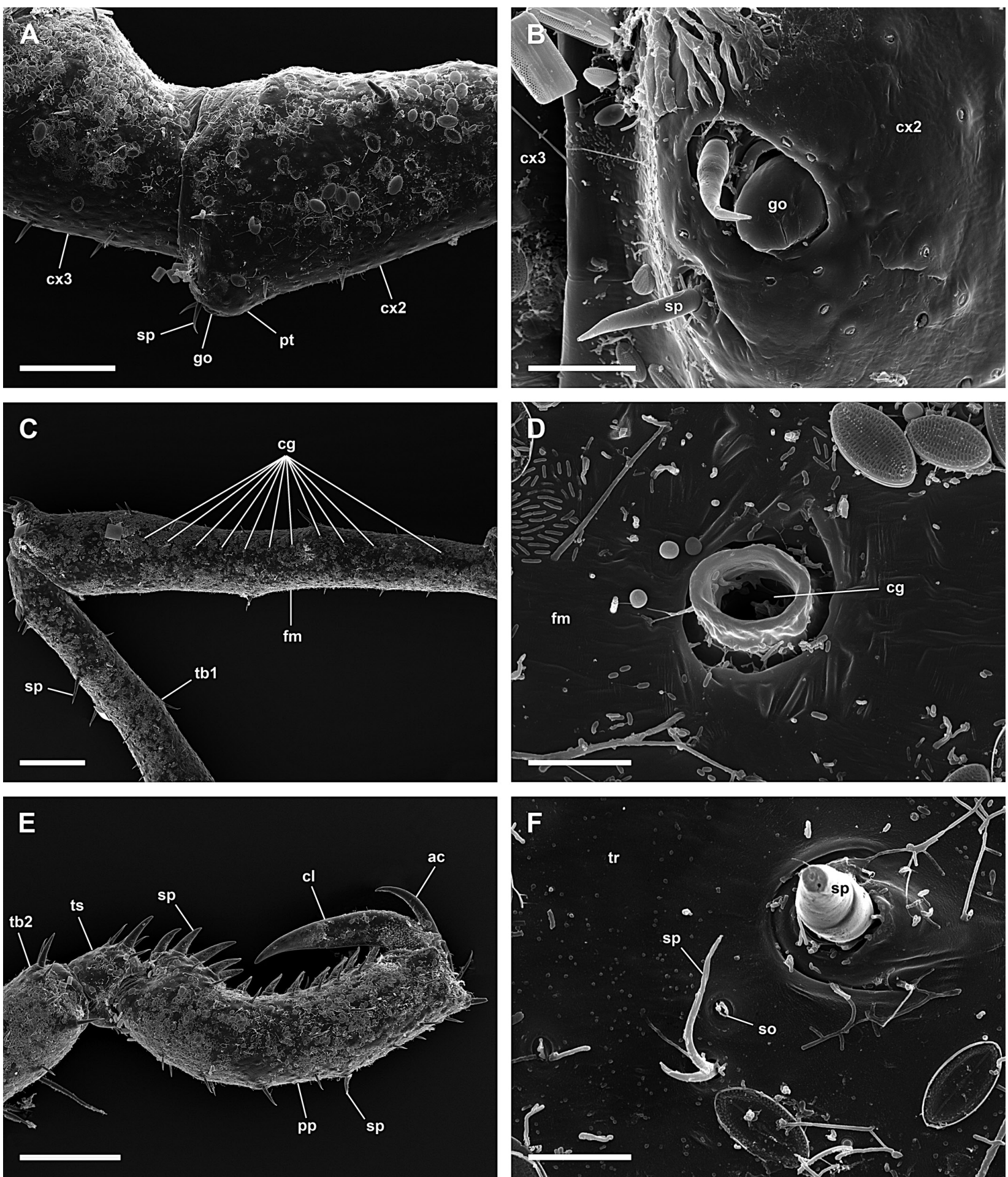

Figure 43. Endeis spinosa, male; A: Lateral view of coxa 2 with genital opening on a protuberance (right 3rd leg); scale $100 \mu \mathrm{m}$; B: Genital opening; scale $20 \mu \mathrm{m}$; C: Femur with overall about 11 cement glands (left 3rd leg); scale $200 \mu \mathrm{m}$; D: Single cement gland; scale $10 \mu \mathrm{m}$; E: Tarsus, propodus, and claws (left 3rd leg); scale $200 \mu \mathrm{m}$; F: Spine, hair and slit organ on dorsal side of trunk; scale $10 \mu \mathrm{m}$. 

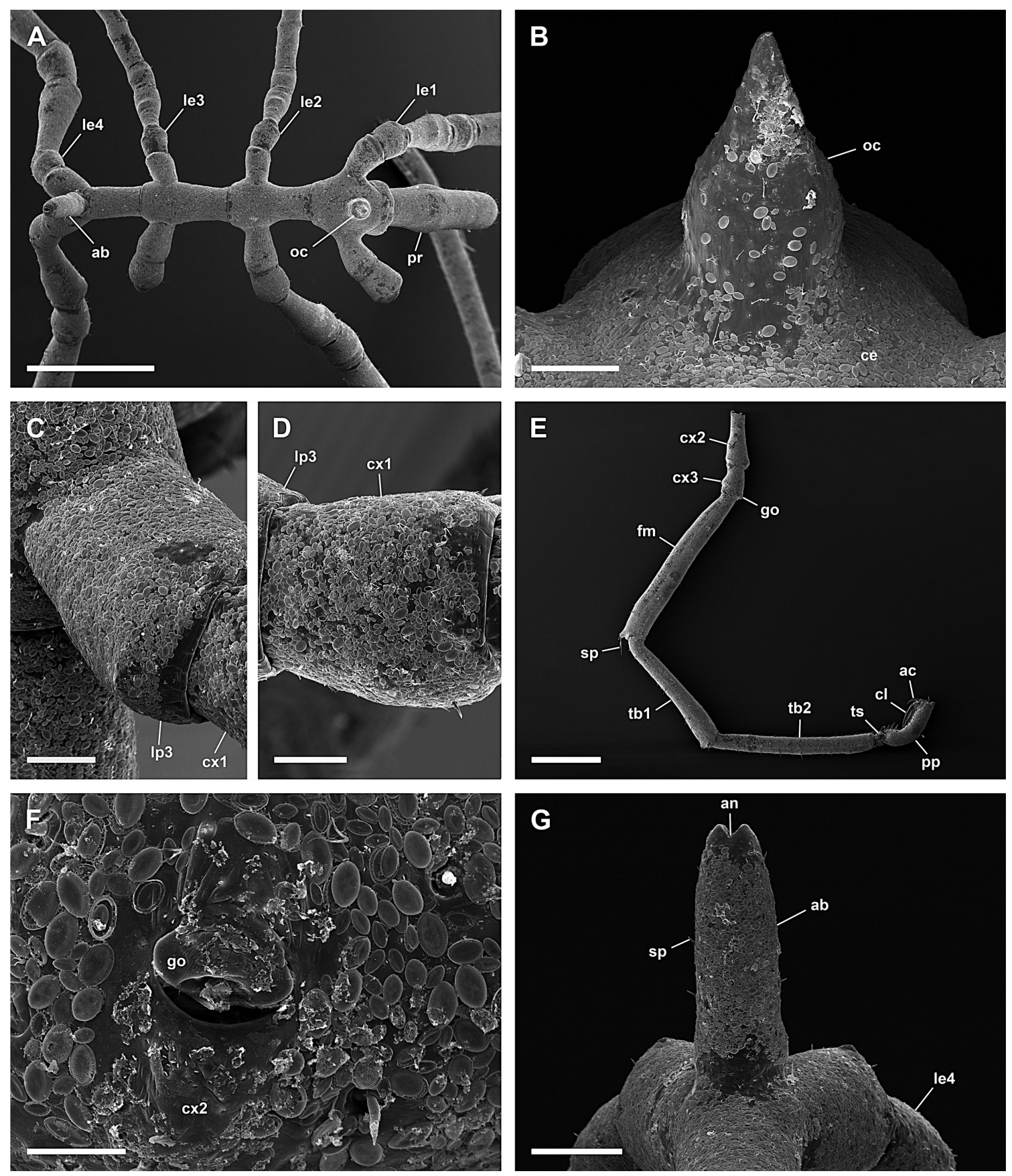

Figure 44. Endeis spinosa, female; A: Dorsal view of trunk; scale $1 \mathrm{~mm}$; B: Ocular tubercle; scale $100 \mu \mathrm{m}$; C: Right lateral process 3 without protuberance; scale $100 \mu \mathrm{m}$; D: Coxa 1 of right 3rd leg without protuberance; scale $100 \mu \mathrm{m}$; E: Right 3rd leg; scale $1 \mathrm{~mm}$; F: Genital opening on ventral side of coxa 2; scale $40 \mu \mathrm{m}$; G: Abdomen; scale $200 \mu \mathrm{m}$. 

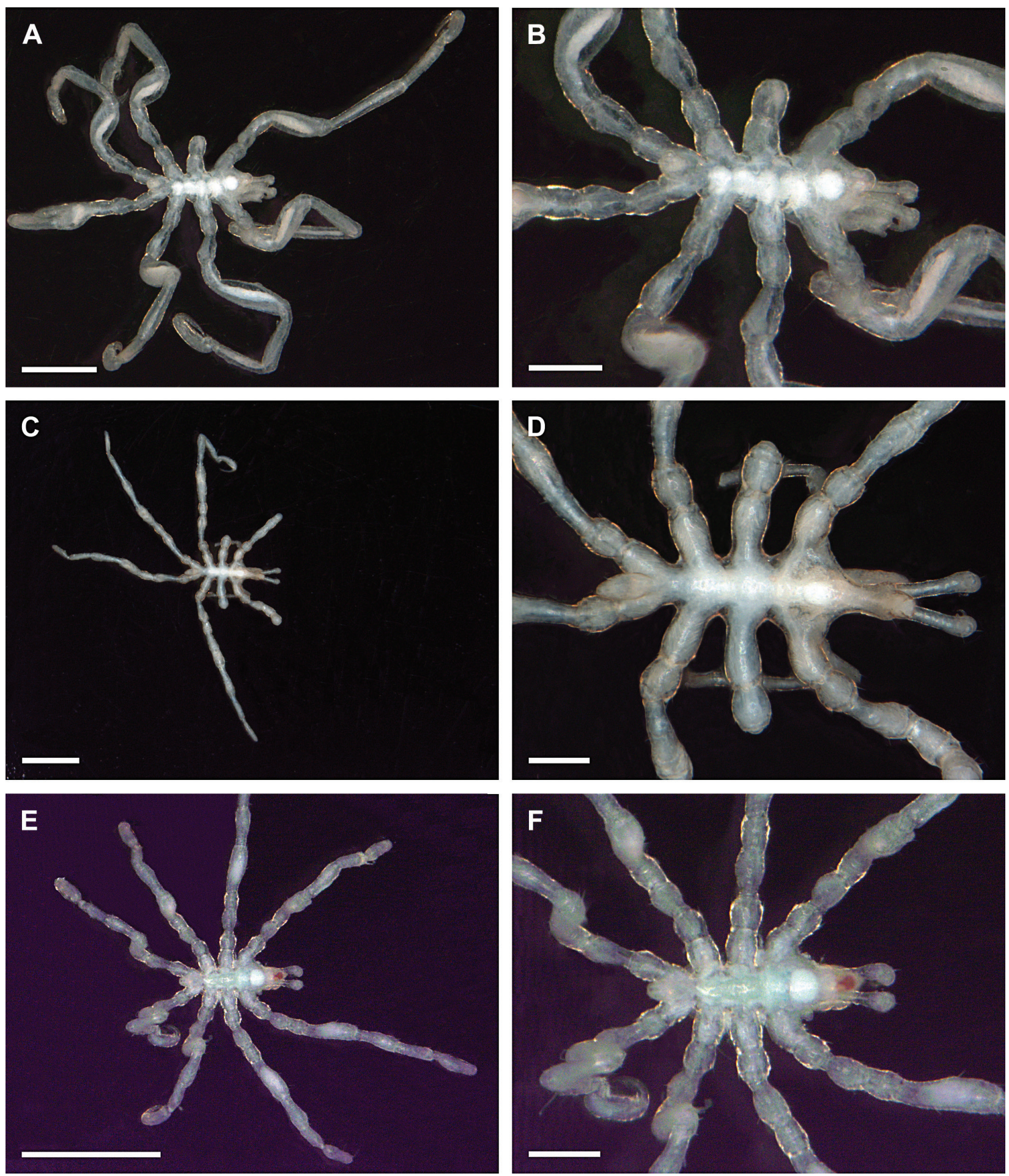

Figure 45. Phoxichilidiidae; A, B: Anoplodactylus angulatus, female, dorsal view; scales $500 \mu \mathrm{m}$ and $250 \mu \mathrm{m}$, respectively; C, D: Anoplodactylus petiolatus, male, dorsal view; scales $1 \mathrm{~mm}$ and $250 \mu \mathrm{m}$, respectively; E, F: Anoplodactylus pygmaeus, male, dorsal view; scales $1 \mathrm{~mm}$ and $250 \mu \mathrm{m}$, respectively. 

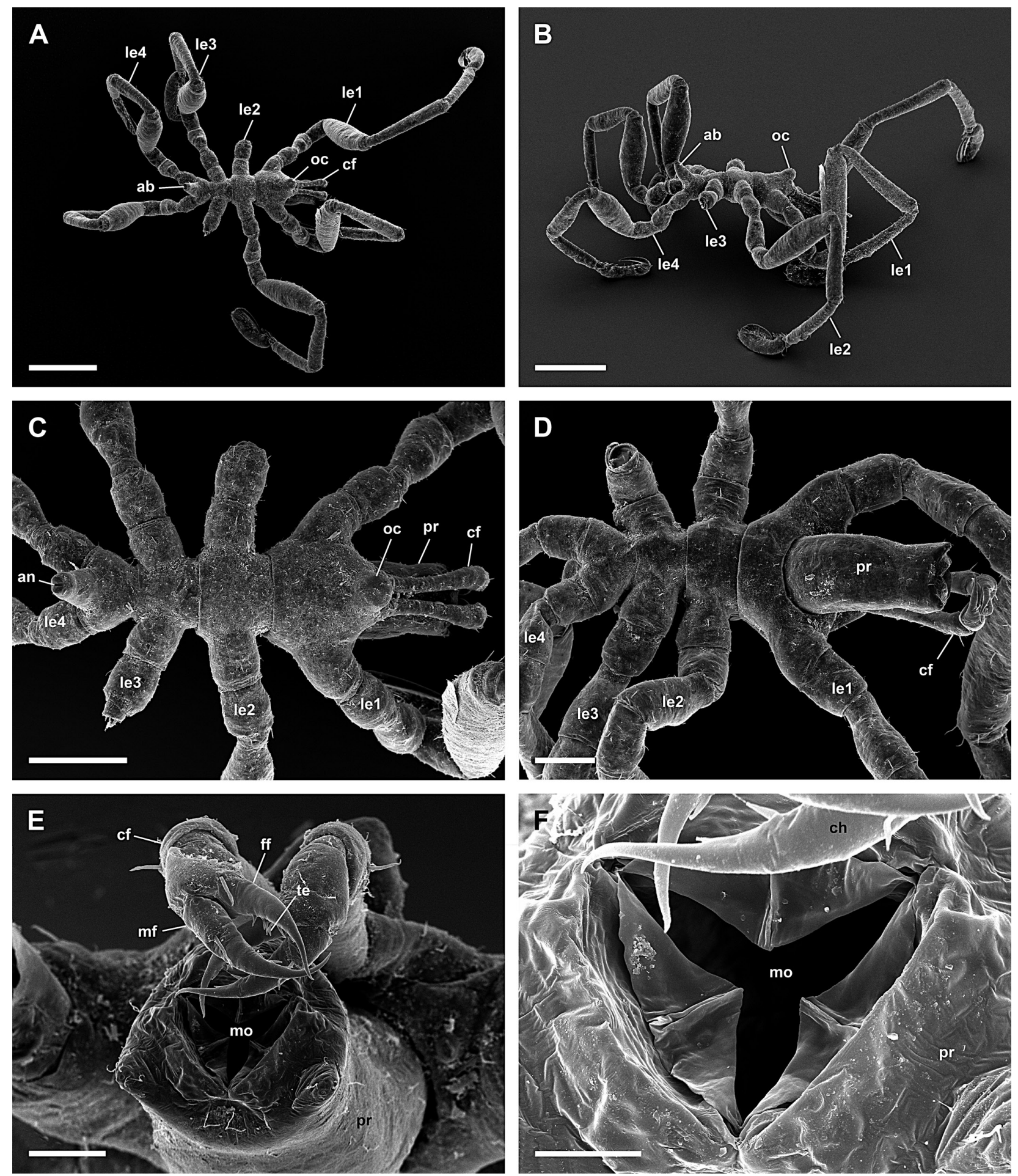

Figure 46 Anoplodactylus angulatus, female; A: Dorsal view; scale $400 \mu \mathrm{m}$; B: Lateral view; scale $400 \mu \mathrm{m}$; C: Dorsal view of trunk; scale $200 \mu \mathrm{m}$; D: Ventral view of trunk; scale $100 \mu \mathrm{m}$; E: Frontal view with chelifores with developed chela and mouth opening; scale $40 \mu \mathrm{m}$; F: Mouth opening; scale $20 \mu \mathrm{m}$. 

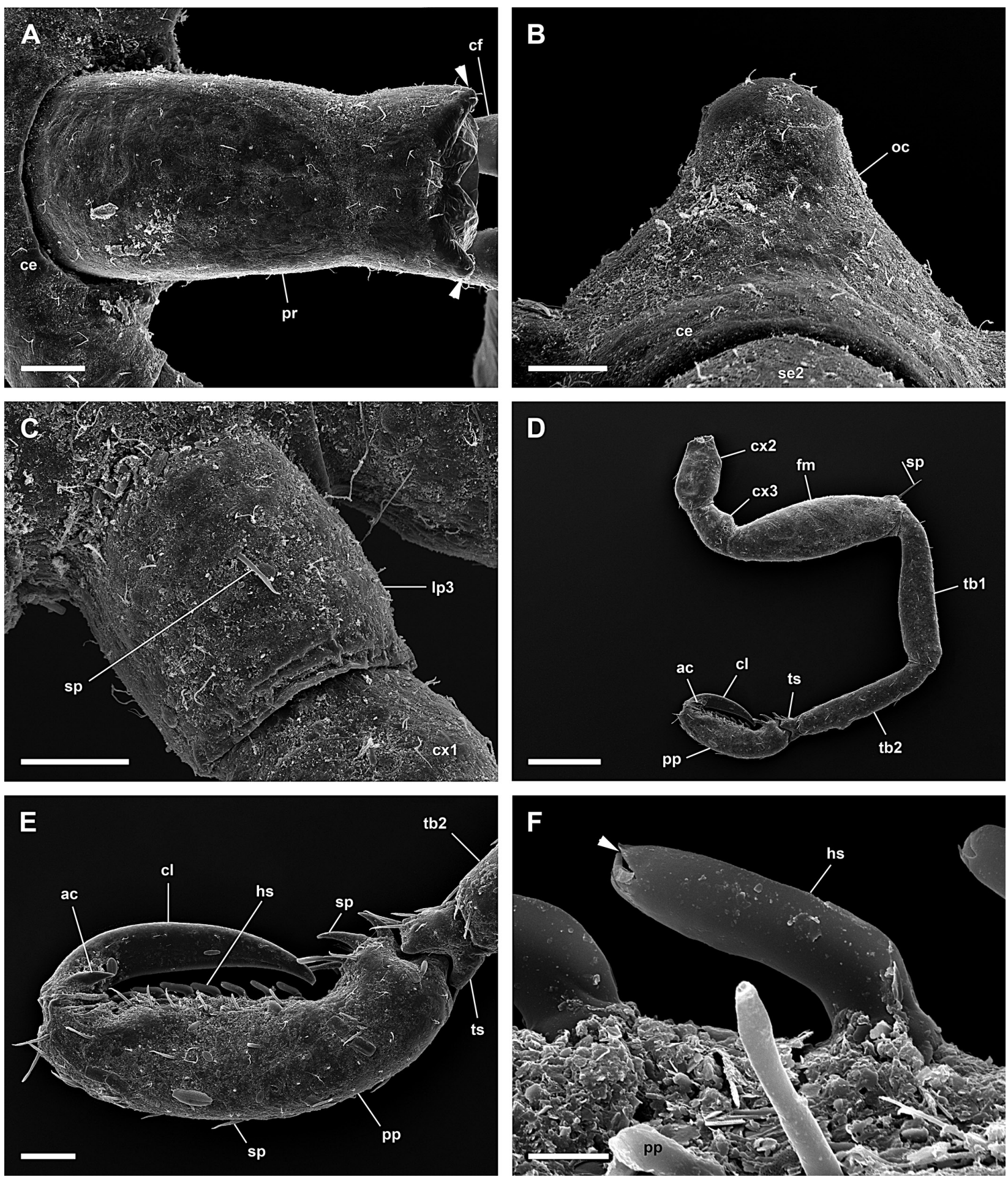

Figure 47. Anoplodactylus angulatus, female; A: Ventral view of proboscis with conspicuous angles at corners (arrowheads); scale $40 \mu \mathrm{m}$; B: Ocular tubercle; scale $40 \mu \mathrm{m}$; C: Right lateral process 3 with spine; scale $40 \mu \mathrm{m}$; D: Right 3rd leg; scale $200 \mu \mathrm{m}$; E: Tarsus and propodus with hollow spine on propodus (right 3rd leg); scale $40 \mu \mathrm{m}$; F: Hollow spine on propodus with slit on tip (arrowhead); scale $5 \mu \mathrm{m}$. 

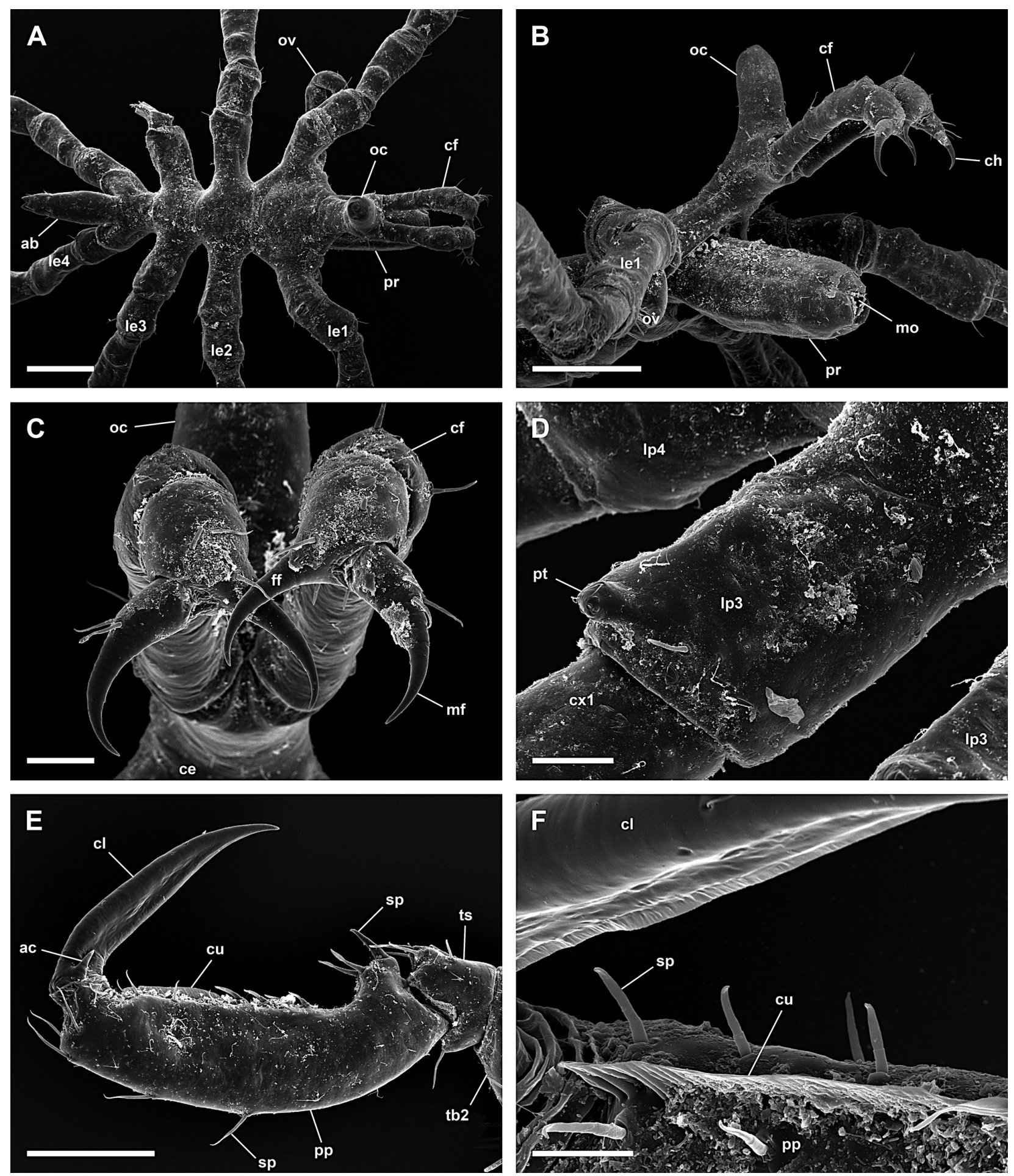

Figure 48. Anoplodactylus petiolatus, male; A: Dorsal view of trunk; scale $200 \mu \mathrm{m}$; B: Lateral view of cephalon overreaching posterior part of proboscis, oviger dissected; scale $200 \mu \mathrm{m}$; C: Chelifores with developed chela; scale $40 \mu \mathrm{m}$; D: Left lateral process 3 with protuberance; scale $40 \mu \mathrm{m}$; E: Tarsus, propodus with cutting lamina, and claws (left 3rd leg); scale $40 \mu \mathrm{m}$; F: Cutting lamina on propodus (right 1st leg); scale $20 \mu \mathrm{m}$. 

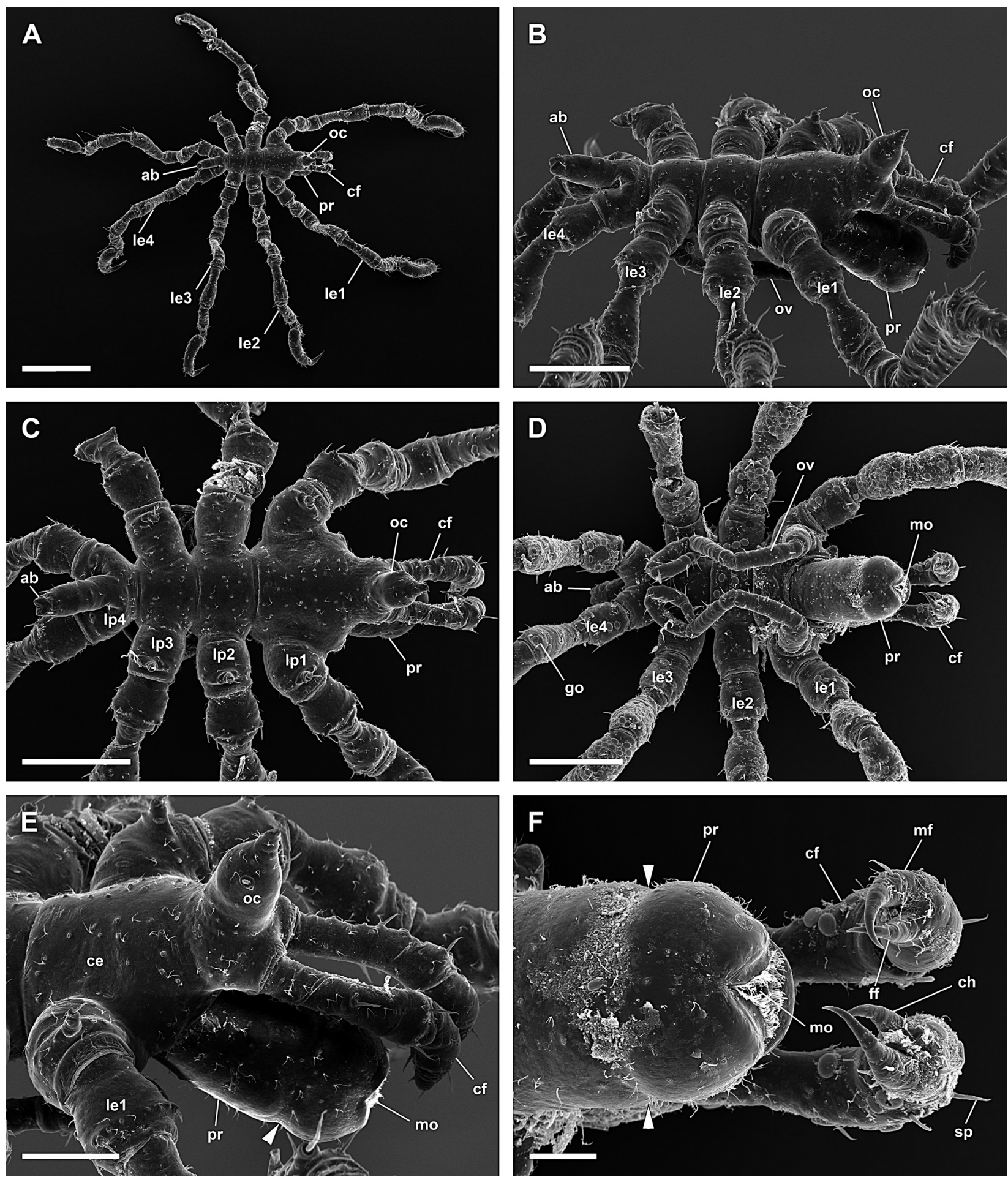

Figure 49. Anoplodactylus pygmaeus, male; A: Dorsal view; scale $400 \mu \mathrm{m}$; B: Lateral view of trunk; scale $200 \mu \mathrm{m}$; C: Dorsal view of trunk; scale $200 \mu \mathrm{m}$; D: Ventral view of trunk; scale $200 \mu \mathrm{m} ; \mathbf{E}$ : Lateral view of cephalon overreaching posterior part of proboscis, proboscis with constriction encircling it subterminally (arrowheads); scale $100 \mu \mathrm{m}$; F: Ventral view of proboscis with constriction encircling it subterminally (arrowheads); scale $40 \mu \mathrm{m}$. 

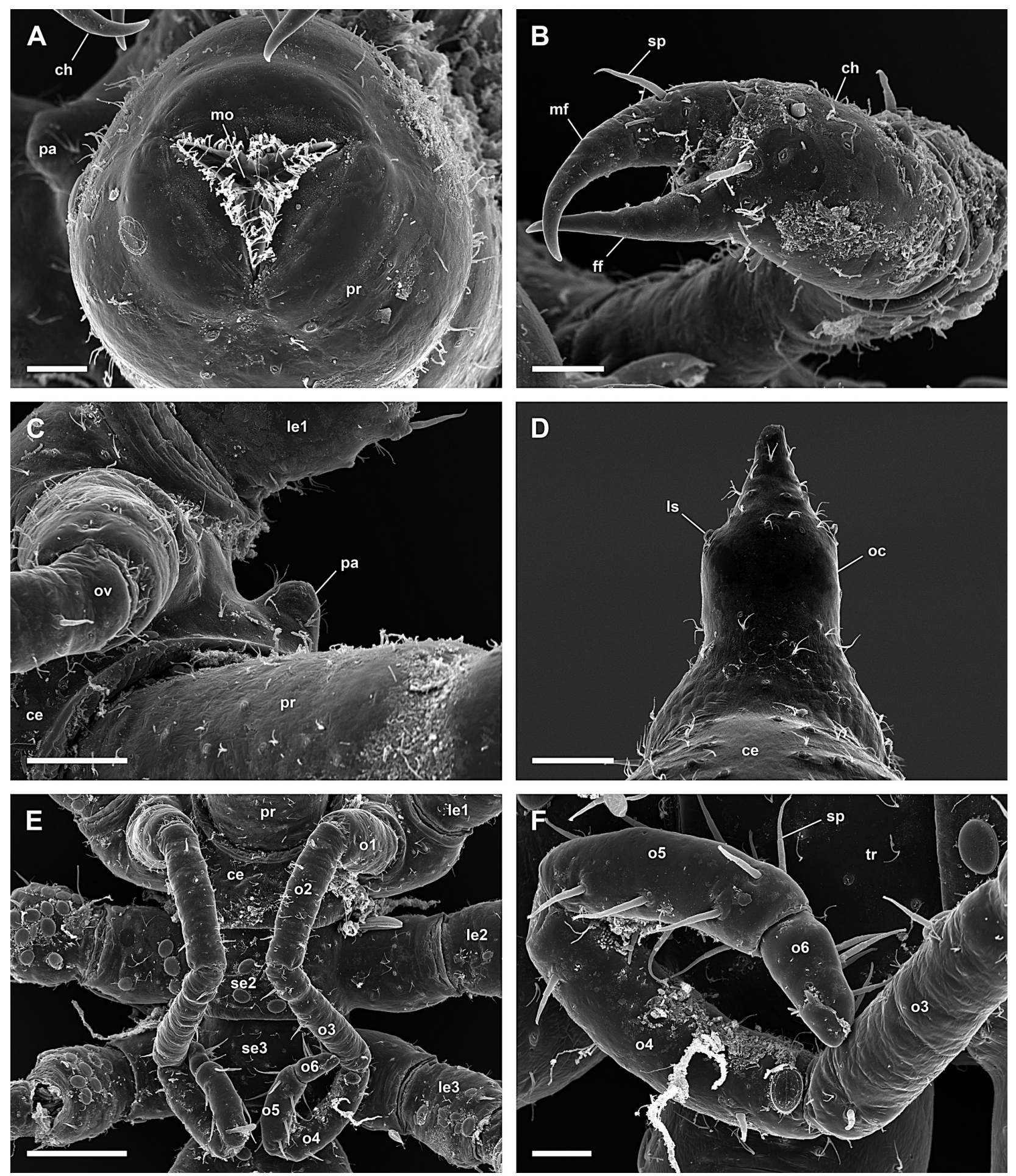

Figure 50. Anoplodactylus pygmaeus, male; A: Mouth opening; scale $20 \mu \mathrm{m}$; B: Left chelifore with developed chela; scale $20 \mu \mathrm{m}$; C: Ventral view of right palp, reduced to a small tubercle; scale $40 \mu \mathrm{m}$; D: Ocular tubercle with lateral sense organ; scale $40 \mu \mathrm{m}$; E: 6-articled oviger; scale $100 \mu \mathrm{m} ; \mathbf{F}$ : Distal articles of left oviger; scale $20 \mu \mathrm{m}$. 

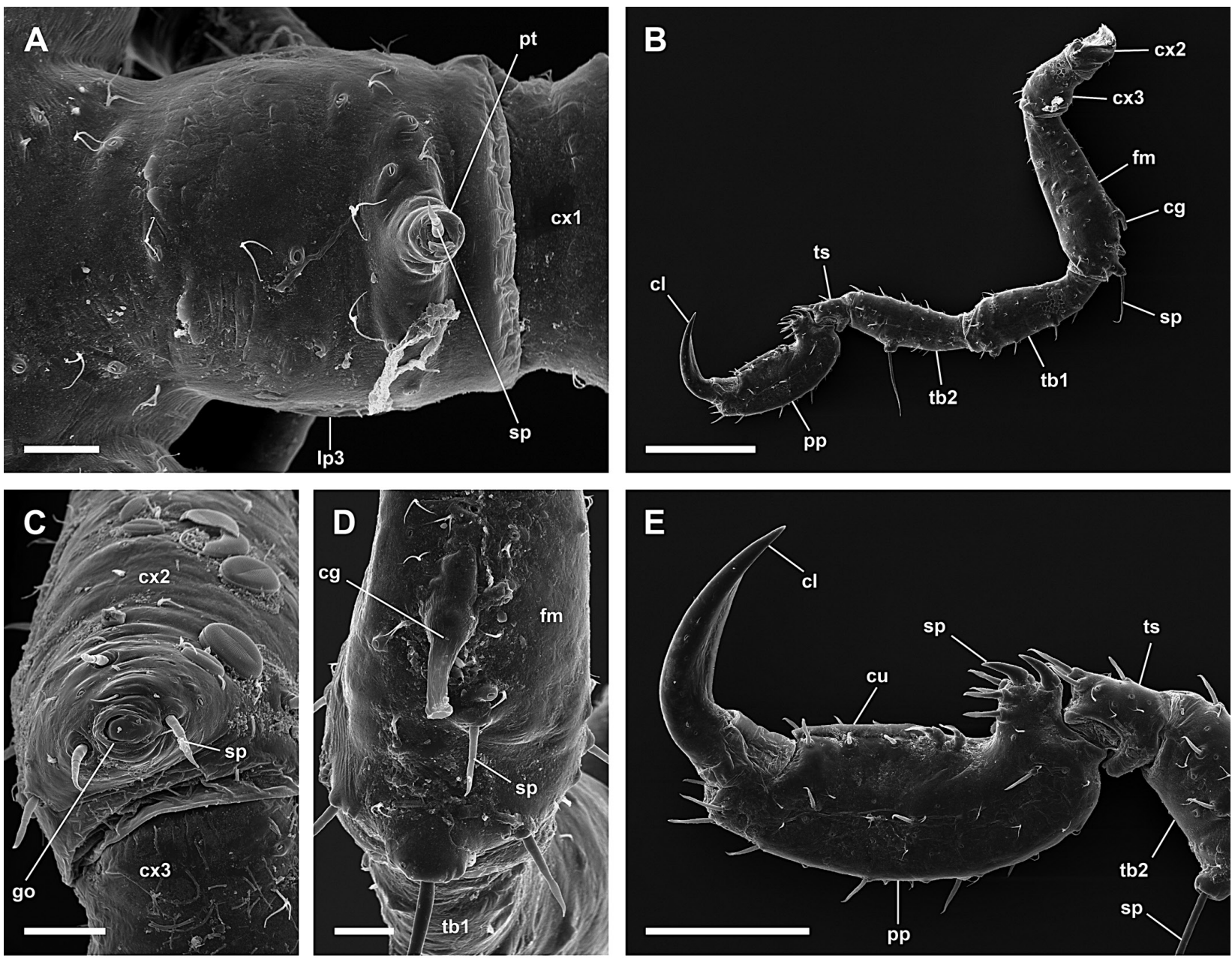

E
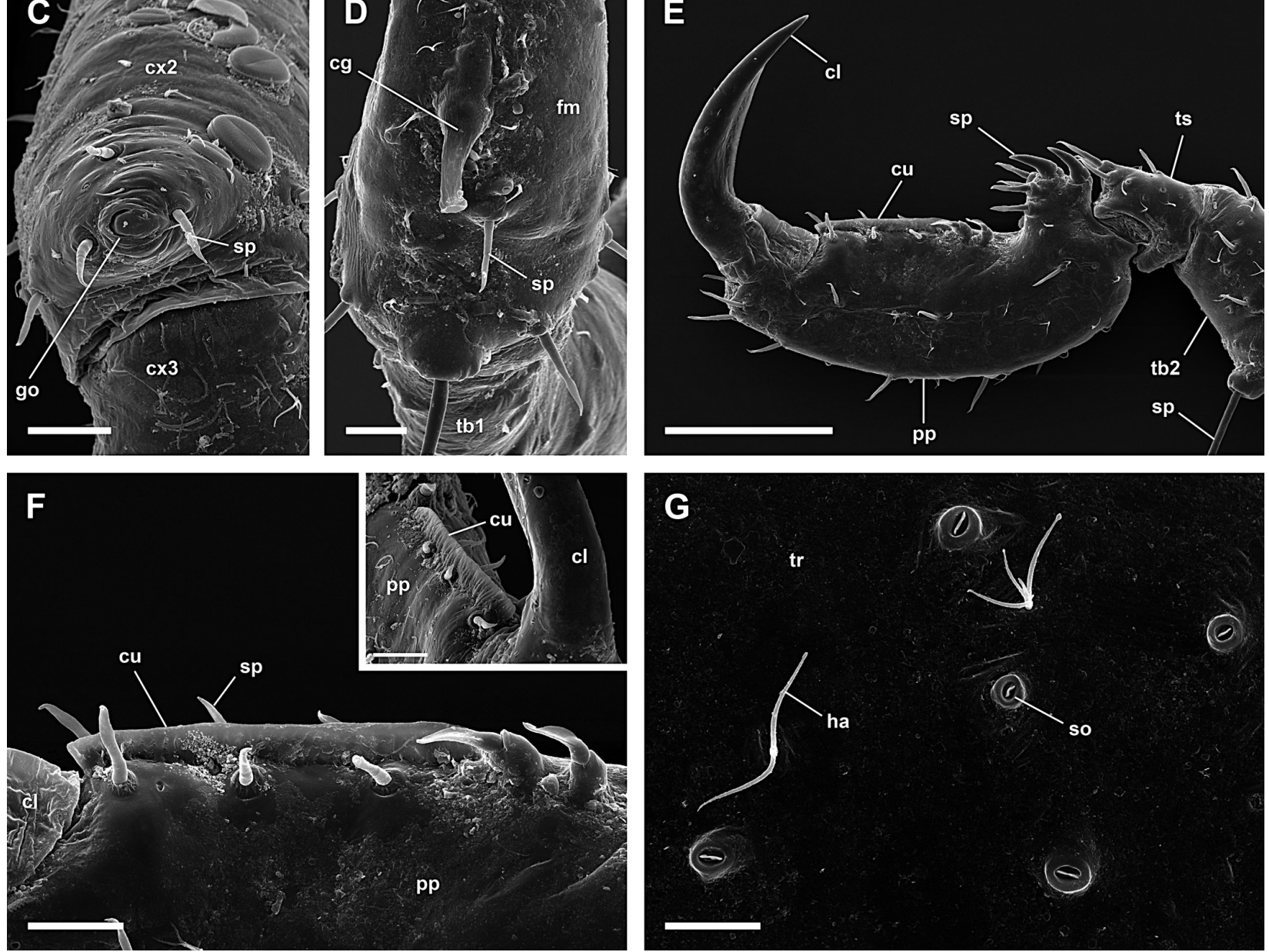

Figure 51. Anoplodactylus pygmaeus, male; A: Right lateral process 3 with protuberance and spine; scale $20 \mu \mathrm{m}$; B: Left 3rd leg; scale $200 \mu \mathrm{m}$; C: Ventral view of coxa 2 with genital opening, distal is down (left 4th leg); scale $20 \mu \mathrm{m}$; D: Dorsal view of femur with cement gland, distal is down (right 4th leg); scale $20 \mu \mathrm{m}$; E: Tarsus, propodus with cutting lamina, and claws (left 3rd leg); scale $100 \mu \mathrm{m} ; \mathbf{F}$ : Cutting lamina on propodus; scale $100 \mu \mathrm{m}$; insert: Detail of cutting lamina; scale $20 \mu \mathrm{m}$; G: Hairs and slit organs on dorsal side of trunk; scale $10 \mu \mathrm{m}$. 

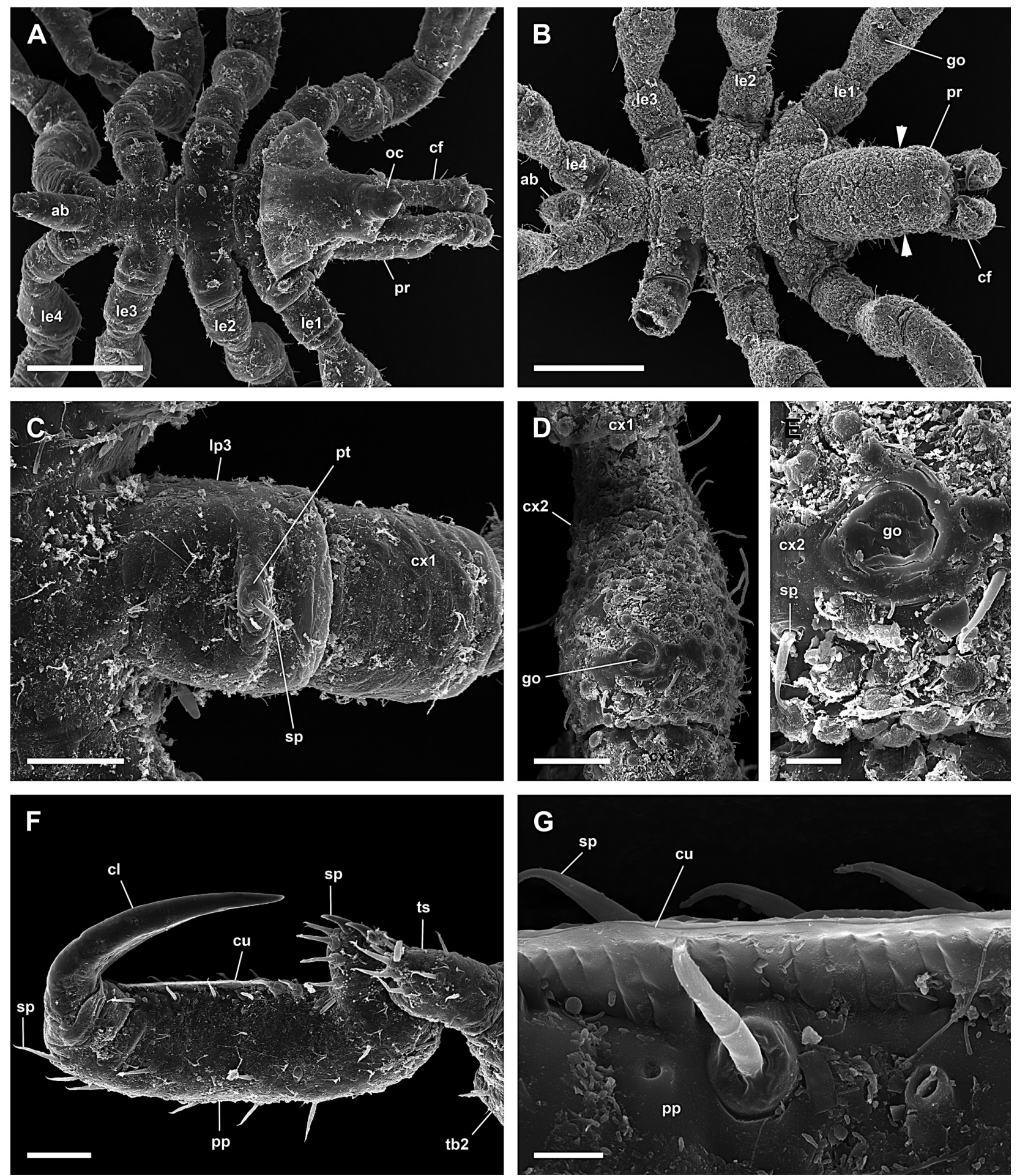

Figure 52. Anoplodactylus pygmaeus, female; A: Dorsal view of trunk; scale $200 \mu \mathrm{m}$; B: Ventral view of trunk, proboscis with a constriction encircling it subterminally (arrowheads); scale $200 \mu \mathrm{m} ; \mathbf{C}$ : Right lateral process 3 with protuberance and spine; scale $40 \mu \mathrm{m}$; D: Ventral view of coxa 2 with genital opening (right 4th leg); scale $40 \mu \mathrm{m}$; E: Genital opening; scale $10 \mu \mathrm{m}$; F: Tarsus, propodus with cutting lamina, and claws (left 1st leg); scale $40 \mu \mathrm{m}$; G: Cutting lamina on propodus; scale $5 \mu \mathrm{m}$. 

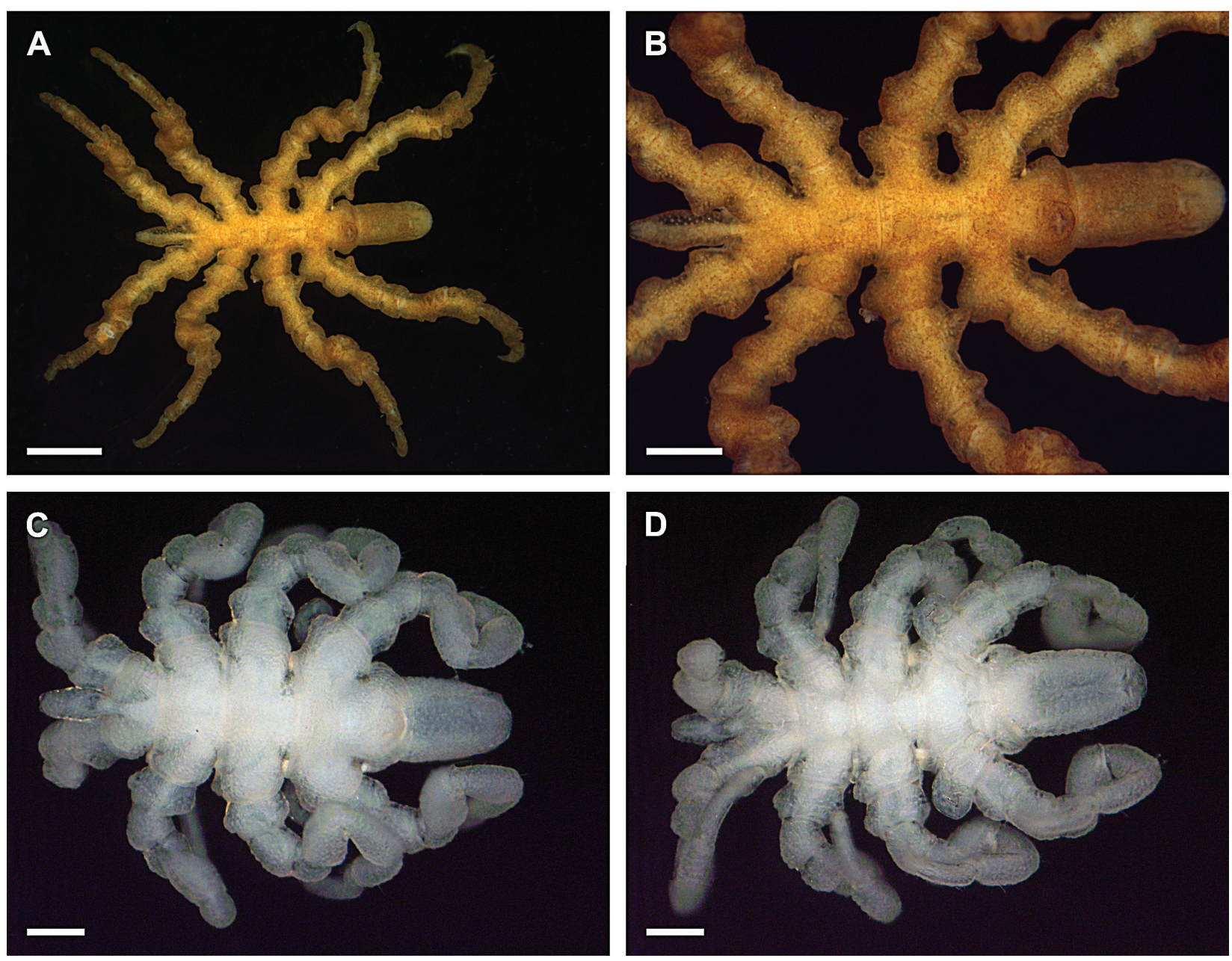

Figure 53. Pycnogonidae; A, B: Pycnogonum nodulosum, female, dorsal view; scales $1 \mathrm{~mm}$ and $500 \mu \mathrm{m}$, respectively; C, D: Pycnogonum (Retroviger) pusillum, male, dorsal and ventral view, respectively; scales $250 \mu \mathrm{m}$. 

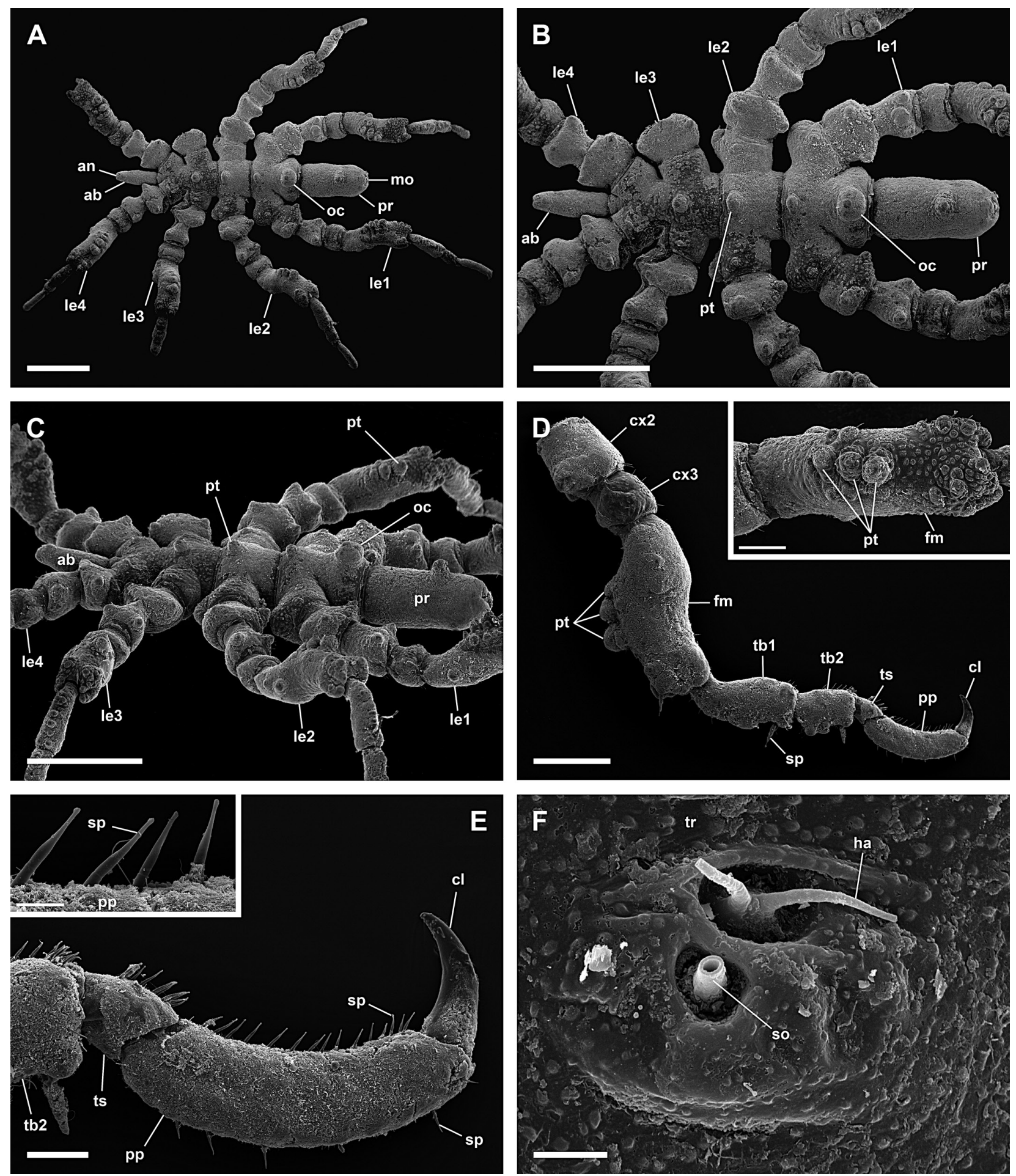

Figure 54. Pycnogonum nodulosum, female; A: Dorsal view; scale $1 \mathrm{~mm}$; B: Dorsal view of trunk with protuberances on dorsal midline; scale $1 \mathrm{~mm}$; C: Lateral view of trunk; scale $1 \mathrm{~mm}$; D: Left 3rd leg with protuberances on femur; scale $400 \mu \mathrm{m}$; insert: Dorsal view of protuberances on femur; scale $200 \mu \mathrm{m}$; E: Tarsus, propodus, and claw without auxiliary claws (left 3rd leg); scale $100 \mu \mathrm{m}$; insert: Spines on propodus; scale $20 \mu \mathrm{m}$; F: Hair and slit organ on dorsal side of trunk; scale $5 \mu \mathrm{m}$. 

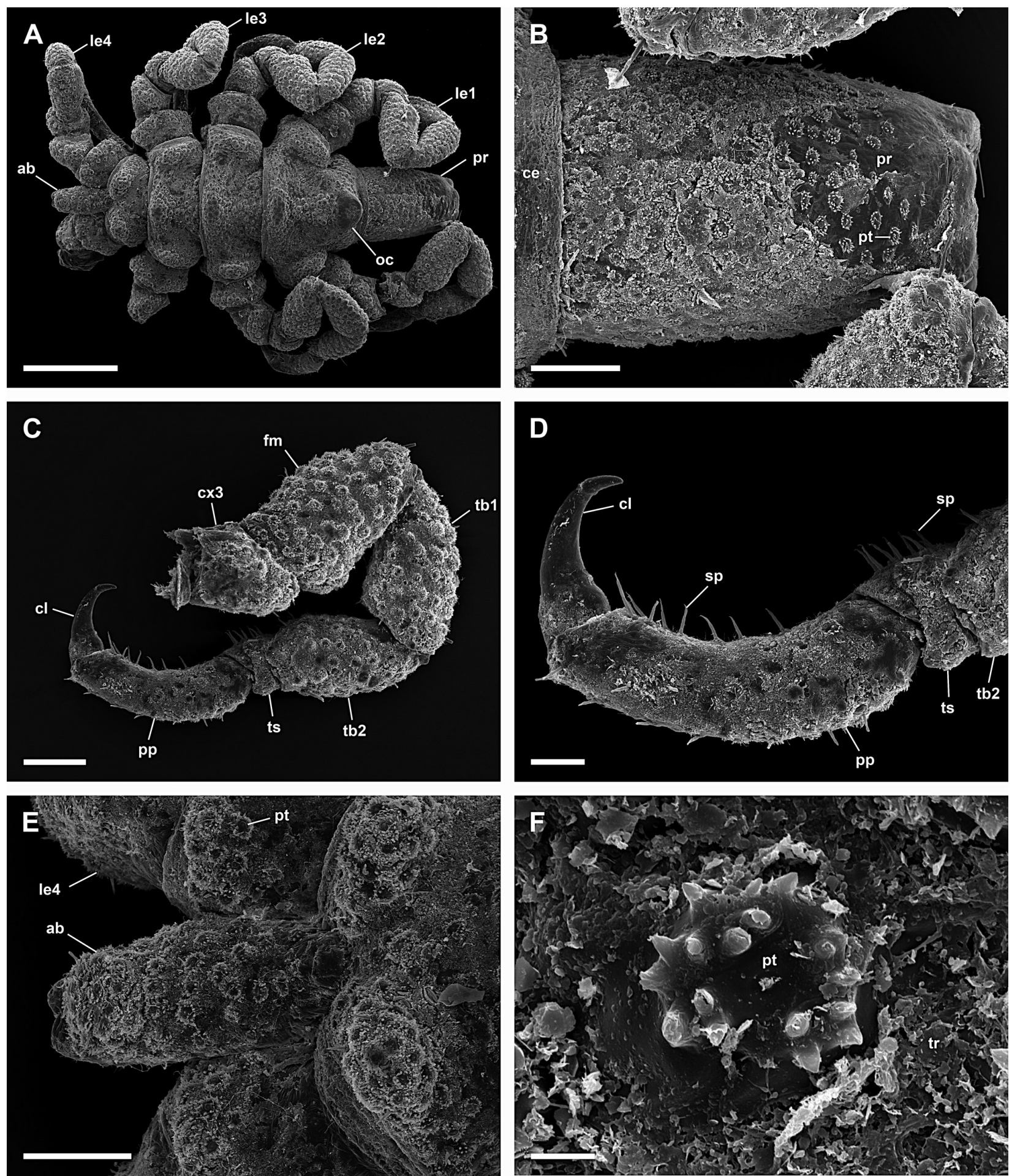

Figure 55. Pycnogonum (Retroviger) pusillum, male; A: Dorsal view; scale $400 \mu \mathrm{m}$; B: Dorsal view of proboscis; scale $100 \mu \mathrm{m}$; C: Right 3rd leg; scale $100 \mu \mathrm{m}$; D: Tarsus, propodus, and claw without auxiliary claws (right 3rd leg); scale $40 \mu \mathrm{m}$; E: Abdomen; scale $100 \mu \mathrm{m} ; \mathbf{F}$ : Protuberance, scattered over trunk and legs; scale $5 \mu \mathrm{m}$. 


\section{Acknowledgements}

We thank Stefan Friedrich for expert technical assistance; Katharina Jörger and Roland Meyer for help with collecting the animals; and Stefan Füssl for help with preparing the figures in Adobe Photoshop.

\section{References}

Arango CP (2002) Morphological phylogenetics of the sea spiders (Arthropoda: Pycnogonida). Organisms Diversity \& Evolution 2: 107-125. doi: 10.1078/1439-6092-00035

Arango CP (2003) Molecular approach to the phylogenetics of sea spiders (Arthropoda: Pycnogonida) using partial sequences of nuclear ribosomal DNA. Molecular Phylogenetics and Evolution 28: 588-600. doi: 10.1016/S1055-7903(03)00069-1

Arango CP, Soler-Membrives A, Miller KJ (2011) Genetic differentiation in the circum-Antarctic sea spider Nymphon australe (Pycnogonida; Nymphonidae). Deep-sea Research Part II: Topical Studies in Oceanography 58: 212-219. doi: 10.1016/j.dsr2.2010.05.019

Arango CP, Wheeler WC (2007) Phylogeny of the sea spiders (Arthropoda, Pycnogonida) based on direct optimization of six loci and morphology. Cladistics 23: 255-293. doi: 10.1111/j.10960031.2007.00143.x

Arnaud F (1987) Les pycnogonides (Chelicerata) de Méditerranée: distribution écologique, bathymetrique et biogéographie. Mesogee 47: 37-58.

Arnaud F, Bamber RN (1987) The Biology of Pycnogonida. Advances in Marine Biology 24: 1-96. doi: 10.1016/S0065-2881(08)60073-5

Bacescu M (1959) Pycnogonides nouveaux pour la foune de la mer Noire: Anoplodactylus petiolatus (Kr.), A. stocki n. sp. and Callipallene brevirostris (John.). Review Roumain de Biologie 4: 117-128.

Bamber RN (2007) A holistic re-interpretation of the phylogeny of the Pycnogonida Latreille, 1810 (Arthropoda). Zootaxa 1668: 295-312.

Bamber RN (2010) Sea-spiders (Pycnogonida) of the north-east Atlantic: Keys and Notes for Identification the Species. Linnean Society of London by Field Studies Council, London, Shrewsbury, 250 pp.

Bamber RN, El Nagar A (2013) Pycnobase: World Pycnogonida Database. Available from: http://www.marinespecies.org/pycnobase/ [accessed March 2014]

Benson DA, Karsch-Mizrachi I, Lipman DJ, Ostell J, Sayers EW (2010) GenBank. Nucleic acids research 38: D46-D51. doi: 10.1093/nar/ gkp1024

Bolte KB (1996) Techniques for obtaining scanning electron micrographs of minute arthropods. Proceedings of the Entomological Society of Ontario 127: 67-87.

Bourdillon A (1954) Contribution à l'étude des pycnogonides de Tunisie. Station océanographique de Salammbo 35: 1-8.

Bouvier EL (1923) Faune de France - Pycnogonides. Paris, 71 pp.

Carapelli A, Torricelli G, Nardi F, Frati F (2013) The complete mitochondrial genome of the Antarctic sea spider Ammothea carolinensis (Chelicerata; Pycnogonida). Polar Biology: 1-10.

Chimenz-Gusso C, Lattanzi L (2003) Mediterranean Pycnogonida: faunistic, taxonomical and zoogeographical considerations. Biogeographia 24: 251-262.

Chimenz C, Tosti M, Cottarelli V (1993) Taxonomical and ecological observations on Pycnogonida from Apulian coasts (Southern Italy). Italian Journal of Zoology 60: 339-347.
Chimenz Gusso C (2000) Picnogonidi delle coste italiane: quadro delle conoscenze. Memorie della Società entomologica italiana 78: 541-574.

Costa OG (1861) Microdoride Mediterranea, o descrizione de'poco ben conosciuti od affatto ignoti viventi minuti e microscopici del Mediterraneo: I. Stamperia dell'Iride, Napoli, $80 \mathrm{pp}$.

Dietz L, Krapp F, Hendrickx ME, Arango CP, Krabbe K, Spaak JM, Leese F (2013) Evidence from morphological and genetic data confirms that Colossendeis tenera Hilton, 1943 (Arthropoda: Pycnogonida), does not belong to the Colossendeis megalonyx Hoek, 1881 complex. Organisms Diversity \& Evolution 13: 151-162. doi: 10.1007/s13127-012-0120-4

Dietz L, Mayer C, Arango CP, Leese F (2011) The mitochondrial genome of Colossendeis megalonyx supports a basal position of Colossendeidae within the Pycnogonida. Molecular Phylogenetics and Evolution 58: 553-558. doi: 10.1016/j.ympev.2010.12.016

Dohrn A (1881) Die Pantopoden des Golfes von Neapel und der angrenzenden Meeresabschnitte. Monographie der Fauna und Flora des golfes von Neapel 3: 1-252.

Dunlop JA, Arango CP (2005) Pycnogonid affinities: a review. Journal of Zoological Systematics and Evolutionary Research 43: 8-21. doi: 10.1111/j.1439-0469.2004.00284.x

Dunlop JA, Friederichs A, Krapp F, Ring C (2007) An annotated catalogue of the sea spiders (Pycnogonida, Pantopoda) held in the Museum für Naturkunde der Humboldt-Universität zu Berlin. Zoosystematics and Evolution 83: 43-74. doi: 10.1002/mmnz.200600017

Faraggiana R (1940) Pantopodi del Mare Ligure. Bollettino dei Musei di Zoologia e Anatomia comparata di Torino 48: 145-158.

Giltay L (1929) Quelques pycnogonides des environs de Banyuls (France). Bulletin et Annales de la Société entomologique de Belgique 69: 172-176.

Giribet G, Edgecombe GD (2012) Reevaluating the arthropod tree of life. Annual review of entomology 57: 167-186. doi: 10.1146/annurev-ento-120710-100659

Hedgpeth JW (1947) On the evolutionary significance of the Pycnogonida. Smithsonian miscellaneous Collection 106: 1-53.

Helfer H (1936) The fishery grounds near Alexandria (Egypt). VIII. Pantopoda. Fisheries Research Directorate, Notes and Memoirs 16: 1-6.

Hodge G (1864) XIII. List of the British Pycnogonoidea, with descriptions of several new species. The Annals and Magazine of Natural History 13: 113-117.

King PE (1986) Sea spiders: a revised key to the adults of littoral Pycnogonida in the British Isles. Field Studies 6: 493-516.

Kocak C, Alan N (2013) Pycnogonida (Arthropoda) from Mersin Bay (Turkey, eastern Mediterranean). Turkish Journal of Zoology 37: 367-371.

Kocak C, Katagan T, Sukatar A (2010) New records of shallow-water sea spiders (Arthropoda: Pycnogonida) from Turkey. Zoology in the Middle East 49: 118-120. doi: 10.1080/09397140.2010.10638403

Krabbe K, Leese F, Mayer C, Tollrian R, Held C (2010) Cryptic mitochondrial lineages in the widespread pycnogonid Colossendeis megalonyx Hoek, 1881 from Antarctic and Subantarctic waters. Polar Biology 33: 281-292. doi: 10.1007/s00300-009-0703-5

Krapp F (1973) Pycnogonida from Pantelleria and Catania, Sicily. Beaufortia 21: 55-74.

Krapp F, Kocak C, Katagan T (2008) Pycnogonida (Arthropoda) from the eastern Mediterranean Sea with description of a new species of Anoplodactylus. Zootaxa 1686: 57-68.

Krøyer H (1844) Bidrag til Kundskab om Pycnogoniderne eller Søspindlerne. Naturhistorisk Tidsskrift, Kjøbenhavn 1: 90-139, Taf. 131. 
Mahon AR, Arango CP, Halanych KM (2008) Genetic diversity of Nymphon (Arthropoda: Pycnogonida: Nymphonidae) along the Antarctic Peninsula with a focus on Nymphon australe Hodgson 1902. Marine Biology 155: 315-323. doi: 10.1007/s00227-008-1029-5

Montagu G (1808) II. Description of several Marine Animals found on the South Coast of Devonshire. Transactions of the Linnean Society of London 9: 81-114. doi: 10.1111/j.1096-3642.1818.tb00327.x

Montoya Bravo MF, Meltzer L, Meyer R, Melzer RR (2006) Pycnogonids under infralitoral stones at Cape Savudrija, Northern Adriatic Sea (Pantopoda, Ammotheidae). Spixiana 29: 87.

Müller HG (1993) World catalogue and bibliography of the recent Pycnogonida. H.-G. Müller, 410 pp.

Munilla T (1993) Pycnogonids from southern Spain: Fauna 1 Project. Journal of the Marine Biological Association of the United Kingdom 73: 543-543. doi: 10.1017/S0025315400033099

Munilla T, Nieto D (1999) Littoral pycnogonids from the Chafarinas islands (Alboran Sea, western Mediterranean). Vie et Milieu 49: $155-161$.

Nakamura K, Kano Y, Suzuki N, Namatame T, Kosaku A (2007) 18S rRNA phylogeny of sea spiders with emphasis on the position of Rhynchothoracidae. Marine Biology 153: 213-223. doi: 10.1007/ s00227-007-0803-0

Nielsen JF, Lavery S, Lörz A (2009) Synopsis of a new collection of sea spiders (Arthropoda: Pycnogonida) from the Ross Sea, Antarctica. Polar Biology 32: 1147-1155. doi: 10.1007/s00300-009-0611-8

Pérez-Ruzafa A, Munilla T (1992) Pycnogonid ecology in the Mar Menor (Murcia, SW Mediterranean). Scientia Marina 56: 21-28.

Ratnasingham S, Hebert PDN (2007) BOLD: The Barcode of Life Data System (http://www.barcodinglife.org). Molecular ecology notes 7: 355-364. doi: 10.1111/j.1471-8286.2007.01678.x

Regier JC, Shultz JW, Zwick A, Hussey A, Ball B, Wetzer R, Martin JW, Cunningham CW (2010) Arthropod relationships revealed by phylogenomic analysis of nuclear protein-coding sequences. Nature 463: 1079-1083. doi: 10.1038/nature08742

Ruppert EE, Fox RS, Barnes RD (2004) Invertebrate zoology: a functional evolutionary approach. Thomson-Brooks/Cole, Belmont, 963 pp.

Sars GO (1888) Pycnogonida borealia et arctica enumerat (Prodromus descriptionis). Archiv for Mathematik og Naturvidenskab, Oslo 12: $339-256$.
Schmidt I, Glaubrecht M, Golani D (2001) Biogeographie und Biodiversität. In: Hofrichter R (Ed.) Das Mittelmeer, Band 1, Allgemeiner Teil: Fauna, Flora, Ökologie. Spektrum, Akad. Verlag, Heidelberg, Berlin, 608.

Schüller S (1989) Die Pantopodenfauna von Rovinj (Nördliche Adria) und der Jahreszyklus einiger Arten. Bonner Zoologische Beiträge 40: 285-295.

Stock JH (1952) Revision of the European representatives of the genus Callipallene Flynn, 1929 (Pycnogonida). Beaufortia 13: 1-15.

Stock JH (1958) Pycnogonida from the Mediterranean coast of Israel. The Bulletin of the Research Council of Israel 7B: 137-142.

Stock JH (1962) Some Turkish pycnogonid records. Entomologische Berichten 22: 218-219.

Stock JH (1966) Sur quelques Pycnogonides de la région de Banyuls (3e note). Vie et Milieu 17: 407-417.

Stock JH (1968) Faune marine des Pyrenees-Orientales, Fascicule 6, Pycnogonides. Vie et Milieu Supplément 19: 1-38.

Stock JH (1987) Faunistic transit between the Atlantic and the Mediterranean: the deep-water Pycnogonida. Cahiers de Biologie Marine 28: 505-519.

Stock JH, Soyer J (1965) Sur quelques Pycnogonides rares de Banyulssur-Mer. Vie Milieu 16: 415-422.

Tichy G, Tomek C, Hsü KJ, Hofrichter R (2001) Geologie und Entstehungsgeschichte. In: Hofrichter R (Ed.) Das Mittelmeer, Band 1, Allgemeiner Teil: Fauna, Flora, Ökologie. Spektrum, Akad. Verlag, Heidelberg, Berlin, 608.

Weis A, Friedrich S, Melzer RR (2011) Antarctic Pycnogonida housed at the Bavarian State Collection of Zoology. Zoosystematics and Evolution 87: 297-317. doi: 10.1002/zoos.201100008

Weis A, Melzer RR (2012a) Chilean and Subantarctic Pycnogonida collected by the "Huinay Fjordos" Expeditions 2005-2011. Zoosystematics and Evolution 88: 185-203. doi: 10.1002/zoos.201200016

Weis A, Melzer RR (2012b) How did sea spiders recolonize the Chilean fjords after glaciation? DNA barcoding of Pycnogonida, with remarks on phylogeography of Achelia assimilis (Haswell, 1885). Systematics and Biodiversity 10: 361-374. doi: $10.1080 / 14772000.2012 .716462$

Zavodnik D (1968) Beitrag zur Kenntnis der Asselspinnen (Pantopoda) der Umgebung von Rovinj (Nördl. Adria). Thalassia Jugoslavica 4: 45-53. 